\title{
STUDIES OF WATER DIFFUSION IN THE VICINITY OF SINGLE-SUPPORTED LIPID MEMBRANES
}

\author{
A Thesis presented to \\ the Faculty of the Graduate School \\ at the University of Missouri
}

In Partial Fulfillment

of the Requirements for the Degree

Doctor of Philosophy

by

ANDREW MISKOWIEC

Dr. Haskell Taub, Thesis Supervisor

MAY 2014 
The undersigned, appointed by the Dean of the Graduate School, have examined the dissertation entitled:

\section{STUDIES OF WATER DIFFUSION IN THE VICINITY OF SINGLE-SUPPORTED LIPID MEMBRANES}

presented by Andrew Miskowiec, a candidate for the degree of Doctor of Philosophy and hereby certify that, in their opinion, it is worthy of acceptance.

Dr. Haskell Taub

Dr. Gavin King

Dr. Paul Miceli

Dr. Ioan Kosztin

Dr. Jason Cooley 


\section{ACKNOWLEDGMENTS}

I would like to thank my advisor, Dr. Haskell Taub, for his advice, guidance,

and patience throughout the duration of my graduate studies at the University of Missouri - Columbia.

I would also like to thank Dr. Mengjun Bai and Dr. Flemming Hansen for their helpful discussions and advice over the course of the last several years. I would also like to thank Dr. Eugene Mamontov, Dr. Ken Herwig, Dr. Souleymane Diallo, Dr. Madhusudan Tyagi, and Dr. Timothy Jenkins at the Spallation Neutron Source at Oak Ridge National Laboratory and the NIST Center for Neutron Research for their assistance during experiments. 


\section{TABLE OF CONTENTS}

ACKNOWLEDGMENTS ................ ii

LIST OF TABLES $\ldots \ldots \ldots \ldots \ldots \ldots$ vi

LIST OF FIGURES $\ldots \ldots \ldots \ldots \ldots \ldots$ vii

ABSTRACT ....................

1 Introduction to Lipid Bilayers and Quasielastic Neutron Scattering 1

1.1 Supported Lipid Bilayer as a Model System . . . . . . . . . . . 4

1.1.1 Lipid Molecules . . . . . . . . . . . . . . . . . . 6

1.1.2 The Gel-to-Fluid Phase Transition for Supported Lipids $\ldots \quad$. 7

1.1.3 Properties of Anionic Lipid Bilayers . . . . . . . . . . . . 9

1.2 Neutron Scattering Background . . . . . . . . . . . . . . . 12

1.2.1 Incoherent Neutron Scattering . . . . . . . . . . . . . 12

1.2.2 The Dynamic Structure Factor _. . . . . . . . . . . . 14

1.2 .3 Elastic Neutron Scattering . . . . . . . . . . . . . . . . 17

1.2.4 Energy Resolution for Backscattering Spectrometers . . . . . . 19

1.3 Modeling Water Diffusion . . . . . . . . . . . . . . 21

1.3.1 Einstein Model of Brownian Diffusion . . . . . . . . . . . . 21

1.3.2 Jump Diffusion Model . . . . . . . . . . . . . . . . . . . . 23

1.3.3 Neutron Scattering from Supercooled Water . . . . . . . . . 24

1.4 Quasielastic Scattering from Lipid Membranes . . . . . . . . . . 27 
1.4.1 Lipid Motions . . . . . . . . . . . . . . . . . . 27

1.4.2 Water Dynamics near Lipid Membranes . . . . . . . . . 28

2 Atomic Force Microscopy on Lipid Bilayers . . . . . . . . . . . 31

2.1 Overview of the AFM Technique . . . . . . . . . . 31

2.2 Sample Preparation for DMPC Layers . . . . . . . . . . . . 33

2.3 Controlling DMPC Bilayer Coverage . . . . . . . . . . . . 34

2.4 Producing Supported DMPG Bilayers . . . . . . . . . . . . . . . 39

2.4.1 Sample Preparation of Supported DMPG Bilayers . . . . . . . 41

2.4.2 Parameter Space for DMPG Bilayer Production . . . . . . . 42

2.4.3 Effect of Divalent Salt Concentration on DMPG Bilayer Formation . . . . . . . . . . . . . . . . . 4 44

2.4.4 Effect of Lipid Concentration on DMPG Bilayer Formation . . 46

2.5 The Gel-to-Fluid Phase Transition for Supported Bilayers . . . . . . . 50

2.6 Complete Bilayers for Neutron Scattering Experiments _. . . . . . 54

3 Neutron Scattering from DMPC Bilayer Lipid Membranes . . . 57

3.1 Introduction $\ldots \ldots \ldots \ldots \ldots \ldots \ldots \ldots \ldots \ldots \ldots \ldots \ldots$

3.2 DMPC Neutron Sample Preparation $\ldots \ldots \ldots \ldots$

3.3 Elastic Neutron Scattering from Supported DMPC Membranes . . . . 62

3.4 Quasielastic Neutron Scattering from Supported DMPC Membranes . 69

3.4.1 Identification of Two Types of Water . . . . . . . . . . . 74

3.4.2 Diffusion Constants of Water _ . . . . . . . . . . 79

3.4.3 Bound Water Motion - A Third Type of Water . . . . . . . 84

3.5 Comparison to Molecular Dynamics Simulations . . . . . . . . . 95 
3.6 Summary - Three Types of Water . . . . . . . . . . . . . . . 99

4 Neutron Scattering from DMPG Lipid Membranes . . . . . . . 102

4.1 Introduction . . . . . . . . . . . . . . . . . . . . 102

4.2 DMPG Neutron Sample Preparation . . . . . . . . . . . . . 103

4.3 Elastic Neutron Scattering from Supported DMPG Membranes . . . . 105

4.3.1 Three Types of Water in DMPG Samples . . . . . . . . . . 109

4.3.2 Melting Behavior of Water in DMPG Membranes . . . . . . 115

4.4 Comparison between DMPC and DMPG . . . . . . . . 117

A Quantifying the Amount of Water in Neutron Scattering Samples of Bilayer Lipid Membranes . . . . . . . . . . . . . 120

B List of Samples Investigated on HFBS and BASIS $\ldots \ldots \ldots 127$

C Theory of Neutron Scattering Cross Sections $\ldots \ldots \ldots$

D Determining the Uncertainty in Diffusion Constants . . . . . 139 BIBLIOGRAPHY . . . . . . . . . . . . . . . 144

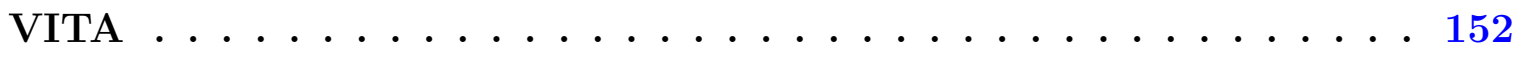




\section{LIST OF TABLES}

Table $\quad$ Page

3.1 Deuteration and water content for DMPC samples . . . . . . . . . 61

4.1 Water content of the DMPG samples . . . . . . . . . . . . . 104

4.2 Water content of DMPG regions . . . . . . . . . . . . 113

A.1 Water content for lipid samples . . . . . . . . . . . . 126

A.2 Water content of the DMPG samples . . . . . . . . . . 126

B.1 List of samples measured on HFBS . . . . . . . . . . . . . . . . . 130

B.2 List of samples measured on BASIS . . . . . . . . . . . . . . 130 


\section{LIST OF FIGURES}

Figure $\quad$ Page

1.1 Methods of supported layer production . . . . . . . . . . . 5

1.2 Structure of the DMPC and DMPG molecules . . . . . . . . . . . 7

1.3 Quasielastic width of supercooled water . . . . . . . . . 26

2.1 Bilayer coverage as a function of lipid concentration . . . . . . . . . 35

2.2 Example of histogram analysis for obtaining bilayer coverage . . . . . 37

2.3 DMPC layer coverage vs. lipid concentration for vesicle fusion . . . . 38

2.4 DMPG morphology at low lipid concentration vs. divalent salt concentration. . . . . . . . . . . . . . . . . 45

2.5 DMPG morphology at moderate lipid concentration vs. divalent salt

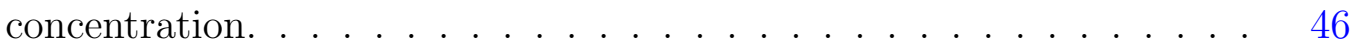

2.6 DMPG morphology at high lipid concentration vs. divalent salt concentration. . . . . . . . . . . . . . . . 47

2.7 DMPG layer coverage vs. concentration. . . . . . . . . . . . . . 48

2.8 DMPG layer coverage vs. concentration. . . . . . . . . . . . . . . 49

2.9 Comparison between DMPC and DMPG step-heights vs. temperature 53

2.10 Comparison between DMPC and DMPG full layers via scratch method 55 
2.11 Comparison between DMPC and DMPG full layers via material dis-

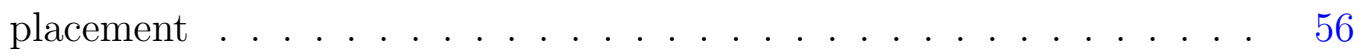

3.1 Sketch of a hydrated single-supported lipid bilayer . . . . . . . . . 59

3.2 Elastic neutron scattering intensity as a function of temperature for DMPC samples of variable hydration. . . . . . . . . . . . . . . 64

3.3 Successive thermal cycles of elastic neutron scattering intensity for a DMPC sample of high hydration collected on HFBS. . . . . . . . 66

3.4 Successive thermal cycles of elastic neutron scattering intensity for a DMPC sample of high hydration collected on BASIS. . . . . . . . 68

3.5 A representative quasielastic spectrum of a high hydration DMPC sam-

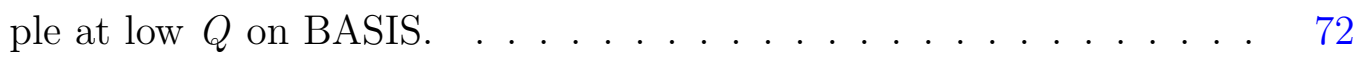

3.6 A representative quasielastic spectrum of a high hydration DMPC sample at high $Q$ on BASIS. . . . . . . . . . . . . . . 73

3.7 Intensities of fitting components for DMPC samples of high and low

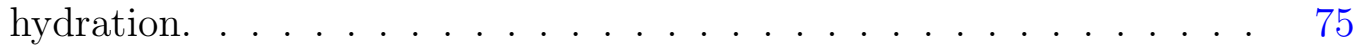

3.8 Width of the broad scattering component as a function of temperature for high- and low-hydration DMPC samples. . . . . . . . . 78

3.9 Width of the broad scattering component as a function of $Q^{2}$ for high and low hydration DMPC samples. . . . . . . . . . . . . . 81

3.10 Diffusion constants of water for high- and low-hydration DMPC samples. 83

3.11 Intensity of the narrow component under different deuteration conditions. 86

3.12 Intensity of the narrow component vs. $Q$ at $272 \mathrm{~K} \ldots \ldots \ldots 8$

3.13 Width of the narrow component for different deuteration conditions. . 92

3.14 Width of the narrow scattering component as a function of temperature 94 
3.15 Water density profile from molecular dynamics simulation . . . . . 97

3.16 Decay rate of selected water molecules in molecular dynamics simulation 98

4.1 Fixed window scans of DMPG bilayers . . . . . . . . . . . . 106

4.2 Effect of annealing on DMPG bilayers . . . . . . . . . . . . . . . 108

4.3 Fixed window scans of DMPG bilayers on cooling . . . . . . . . . . . 110

4.4 Identification of three freezing regions on DMPG bilayers . . . . . . . 112

4.5 Film thickness of water regions in DMPG . . . . . . . . . . . . . 114

4.6 Fixed window scans of DMPG bilayers on heating . . . . . . . . . . 116

A.1 Quasielastic component intensities for alkanes . . . . . . . . . 123

B.1 Fixed window scans of initial DMPC samples. . . . . . . . . . . . 131

B.2 Fixed window scans of low hydration DMPC samples. . . . . . . . . . 132

D.1 $\chi^{2}$ of fit as a function of FWHM of broad component . . . . . . . . . 142

D.2 Parabolic fit to $\chi^{2}$ as a function of FWHM of broad component . . . 143 


\begin{abstract}
The insertion and function of membrane-embedded proteins is one of the most fundamental challenges facing biological physics today. At its core, addressing these phenomena requires an understanding of the interaction of proteins with the lipid bilayer and its associated water molecules. As such, the study of water dynamics and structure near model lipid bilayers can provide foundational knowledge upon which more detailed understanding of these core issues may be developed.

Previous quasielastic neutron scattering measurements on lipid membranes have used samples of large stacks of membranes with an unknown amount of water between layers. This geometry complicates interpretation and renders comparison to molecular dynamics simulations difficult. Instead, this work investigates water dynamics on single-supported bilayers of the model charge-neutral lipid DMPC (dimyristoyl-snglycero-3-phosphocholine) and its anionic analogue DMPG (dimyristoyl-sn-glycero3-phosphoglycerol). Single bilayers can be more directly compared to molecular dynamics simulations, can be interrogated with Atomic Force Microscopy, and avoid the uncertainty in quantifying the amount of water in samples.

A new method for producing the anionic bilayers is developed, which is a variant of the vesicle fusion method. Atomic Force Microscopy is used to characterize the quality of both DMPC and DMPG membranes supported on $\mathrm{SiO}_{2}$-coated silicon substrates. Measuring the bilayer thickness as a function of temperature reveals that the gel-tofluid phase transition is found to be shifted to significantly higher temperatures for adsorbed lipid bilayers in air compared to free vesicles in solution.

The temperature-dependent quasielastic spectra from hydrated DMPC bilayers
\end{abstract}


reveal three types of membrane-associated water. First, a large amount of water diffuses similarly to bulk supercooled water and freezes at $265 \mathrm{~K}$. Second, a smaller amount of water closer to the membrane diffuses more slowly than bulk supercooled water at the same temperature and freezes continuously from 265 to $250 \mathrm{~K}$. Third, 8-11 water molecules per lipid diffuse on the same nanosecond timescale as $\mathrm{H}$ atoms within the lipid molecules, suggesting that they are bound to the lipid headgroups, and remain mobile to $250 \mathrm{~K}$.

Water near the anionic membrane behaves qualitatively different than near the neutral lipid. For the DMPG membrane, no evidence of an abrupt bulk-like freezing transition is seen; instead, water freezes continuously down to $200 \mathrm{~K}$. We speculate that this behavior may be caused by the stronger charged headgroup interaction or the presence of counterions in the water. Evidence for this hypothesis includes the complete melting of water near the DMPG membrane below the bulk point for samples regardless of total hydration. 


\section{Chapter 1}

\section{Introduction to Lipid Bilayers and Quasielastic Neutron Scattering}

In this thesis, we study the dynamics of water near two model lipid membranes with quasielastic neutron scattering (QENS). The charge-neutral lipid dimyristoylphosphocholine (DMPC) and the anionic dimyristoylphosphoglycerol (DMPG) will be investigated. To our knowledge, these are the first QENS studies of water associated with single bilayers of lipids adsorbed on oxide-coated silicon wafers. This single-supported bilayer geometry avoids the complication of an unknown amount of water between lipid bilayers in multilayer systems, which have previously been studied. Furthermore, comparison to molecular dynamics simulations is facilitated by the single-bilayer system. A QENS study of the supported anionic lipid bilayer is also new and will be described herein.

Because of experimental difficulties in probing the cell membrane directly, the supported lipid bilayer (SLB) has gained increasing attention in recent years as a model for biological membranes. In Section 1.1, we give the advantages of SLBs 
in order to motivate and justify the use of the SLB for studying the dynamics of membrane-associated water. In this section, we also give a brief overview of how SLBs are fabricated.

In particular, QENS is an ideal tool for studying the diffusion of water near SLBs. In Section 1.2, it will be shown that the time and length scales accessible by backscattering spectrometry (a method of performing a QENS experiment) and the sensitivity of neutrons to hydrogen are well-suited to studying the diffusive motion of water.

Modeling the quasielastic neutron spectra of water, in particular its translational diffusion constant, has been a topic of research focus for decades. Because one of the major results of this dissertation is the extraction of translational diffusion constants of membrane-associated water below the bulk freezing temperature, it is important to understand the limitations of this analysis. Section 1.3 will provide the necessary context.

A major contribution of this work is the use of single-supported lipid bilayers for QENS measurements. Previous neutron scattering literature reports have focused on the use of multilayer stacks of membranes, a necessary procedure to generate signal intensity due to the flux-limited nature of neutron scattering experiments. Section 1.4 will provide an overview on the results of previous multilayer studies both in terms of water motion and lipid motion.

This dissertation is then divided into three experimental chapters. Chapter 2 will discuss the fabrication and characterization by Atomic Force Microscopy (AFM) of the SLBs used in the neutron scattering measurements. Both a neutral and an anionic lipid membrane will be studied with neutron scattering. The first portion of 
Chapter 2 will demonstrate the fabrication of high-quality layers of the neutral lipid. The development of the SLB system for the anionic lipid is new on a silicon oxide substrate and requires a significant revision of the fabrication techniques developed for the neutral lipid in order to achieve high-quality samples. The second portion of Chapter 2 will provide a discussion of the main challenges associated with producing anionic SLBs as well as evidence of high-quality layers.

A strong motivation for the neutron scattering measurements is a molecular dynamics simulation of a free-standing neutral lipid membrane which can probe similar time and length scales as our experiment [1]. However, one of the significant drawbacks of the SLB system is the effect of the substrate on one or possibly both membrane leaflets, a perturbation which is not present for a free-standing membrane. An indirect measure of the magnitude of the substrate interaction can be provided by measuring the membrane's main gel-to-fluid phase transition. The phase transition appears for free vesicles near room temperature, but it is shifted upwards when the lipids are supported. The measurement of the gel-to-fluid phase transition for both model membranes by AFM will be presented in Chapter 2 .

Chapters 3 and 4 will discuss the results of elastic and quasielastic neutron scattering measurements on the supported neutral and anionic membranes, respectively, as a function of sample hydration and deuteration. Chapter 3 will begin with elastic intensity temperature scans designed to investigate the freezing of water. These measurements are followed by full quasielastic spectra in the same temperature range which allow the extraction of two quasielastic components. One component is identified with the translational diffusion of water, which deviates from that of bulk supercooled water, and is analyzed according to the methods discussed in Section 
1.3. The width of the quasielastic scattering is compared to the simulations and the diffusion constant is determined. A second component in the scattering is identified with a slower, bounded motion of water and lipids themselves.

In Chapter 4, elastic scans of the anionic lipid are discussed. The freezing behavior of water near the anionic lipid is found to be significantly different than for the neutral lipid which is speculated to be related to the charged nature of the head group and the different wetting behavior of water to the anionic membrane.

\subsection{Supported Lipid Bilayer as a Model System}

A method for depositing single layers of materials was demonstrated in the 1920s and 1930s by Blodgett and Langmuir [2-4]. The original series of experiments involved the deposition of stearic acid (a simple amphiphilic hydrocarbon) and various metal salts of stearate. By suspending such materials in an aqueous solution, the amphiphilic molecules self-organize at the air-water interface such that the hydrocarbon tails arrange themselves nearly vertically. When a solid support is lifted through the film at the air-water interface, a single monolayer can be transferred to the substrate. A second layer in the reverse orientation can be deposited by pushing the monolayer-coated substrate through the air-water interface. Repeated introduction of the substrate can result in multiple layer formation. This so-called Langmuir-Blodgett method allows nearly perfect control of the layer number and can be used to produce multilayers of arbitrary layer number. For the development of this technique, Langmuir was awarded the Nobel Prize in Chemistry in 1932.

The Langmuir-Blodgett method has since been a staple technique in producing monolayers and bilayers of lipid molecules, which have the same amphiphilic nature 

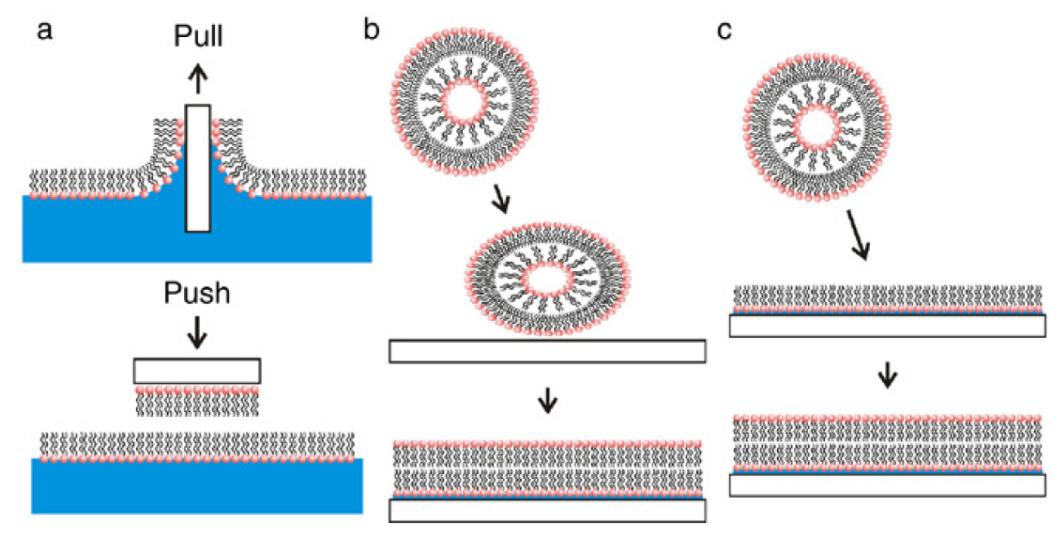

Figure 1.1: (a) The Langmuir-Blodgett method can produce bilayers in two steps: deposition of the bottom leaflet by pulling through the air-water interface and deposition of a second leaflet by pushing down. This variation, wherein the substrate is held horizontally, is called the Langmuir-Schaefer technique and has the advantage that the substrate is not beneath the surface after the bilayer is produced. The vesicle fusion method in (b) and with predeposited monolayer in (c), can also produce uniform bilayers but does not control the lipid number density. From Ref. [6].

as the original stearic acid salts. The first demonstration of a solid-supported lipid bilayer was achieved in 1985 on silica [5]. More recently, it has been realized that a suspension of spherical vesicles may spontaneously adsorb and rupture on a hydrophilic surface in a process known as vesicle fusion. Both the Langmuir-Blodgett and vesicle fusion techniques, depicted in Figure 1.1, have their advantages. The modern application of the Langmuir method involves a Langmuir trough which controls the surface pressure of the suspended film. In this way, the number density and the phase of the solute can be controlled during deposition. On the other hand, the vesicle fusion method allows membrane proteins to be inserted into the system which would otherwise be denatured by the air interface.

Among the substrates that have been demonstrated to support lipid membranes are silica, mica, glass, metal films (indium-tin-oxide, gold, silver, and platinum), and 
a variety of hydrophilic polymers [7-18]. Generally, the surface must be hydrophilic and relatively flat in order for bilayer formation to occur. The concentration of salts in the solution is also known to influence the rate of vesicle adsorption and spreading once on the surface.

Once produced, the supported lipid bilayer offers many experimental advantages over its free-vesicle counterparts. The supported layer is planar, self-assembled, and, for biophysical purposes, can provide the substrate for membrane proteins, cholesterol, and other cellular constituents [19-21]. The planar geometry allows for investigation via AFM and fluorescence techniques and is ideal for reflectivity measurements [22$25]$.

In this dissertation, the vesicle fusion method will be used exclusively to produce samples both for neutron and AFM measurements. While the Langmuir method is useful for producing full lipid layers, it is not suitable for AFM measurements requiring sub-monolayer coverage and it is difficult to scale to a large number of wafers as is required for a quasielastic neutron scattering measurement.

\subsubsection{Lipid Molecules}

The two particular molecules that will be studied in later chapters are the zwitterionic (neutral by two full opposite charges) dimyristoyl-sn-glycero-3-phosphocholine (DMPC) and its anionic analog dimyristoyl-sn-glycero-3-phosphoglycerol (DMPG), shown in Figure 1.2. Both molecules contain two non-polar, fully-saturated aliphatic chains and a glycerol backbone. The lipid head group for both molecules contains a negatively charged phosphate group which is neutralized by a positively charged choline group in DMPC. The DMPG molecule is terminated by a neutral glycerol 


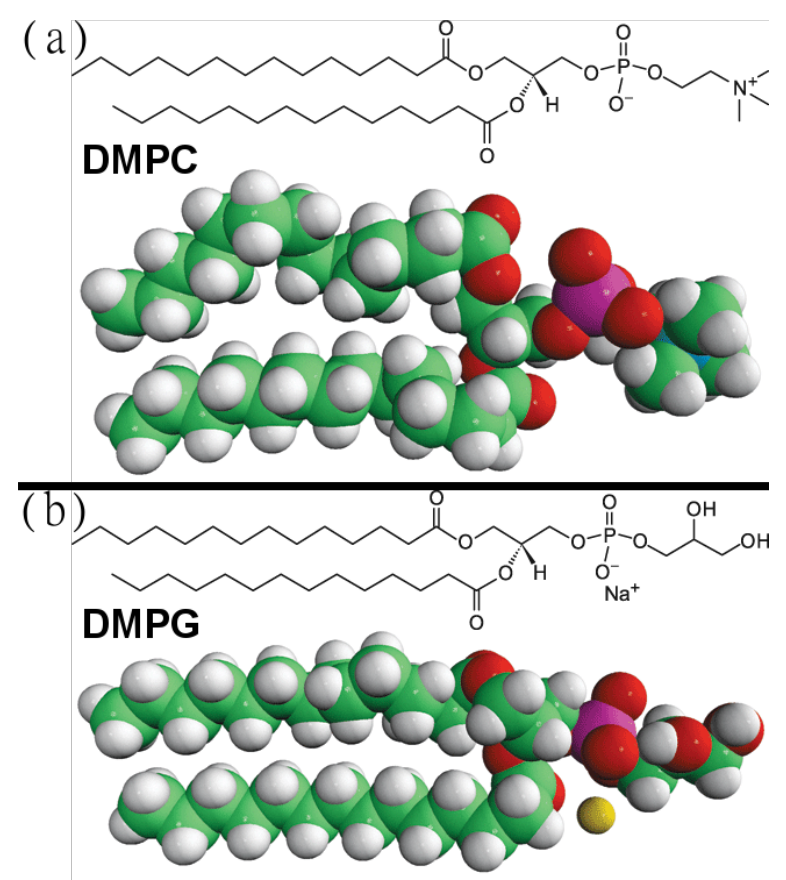

Figure 1.2: Structure of the DMPC $\left(\mathrm{C}_{36} \mathrm{H}_{72} \mathrm{NO}_{8} \mathrm{P}\right)$ and DMPG $\left(\mathrm{C}_{34} \mathrm{H}_{66} \mathrm{O}_{10} \mathrm{PNa}\right)$ molecules. DMPC [panel (a)] is zwitterionic, being neutral by virtue of the $\mathrm{O}^{-}$ and $\mathrm{N}^{+}$. DMPG [panel (b)] is characterized by the neutral glycerol group, which endows the molecule a -1 charge due to the remaining negatively charged phosphate group. In this figure, the phosphate is neutralized by a sodium ion; however, any monovalent salt can provide the neutralizing charge. The two hydrocarbon tails are fully saturated. In the bilayer configuration, two molecules are arranged end-to-end, with their hydrocarbon tails forming a hydrophobic domain while the polar head groups are exposed to the solvent. Structural diagrams from Avanti.

group, thus endowing the molecule with a net negative charge. This negative charge can be neutralized with any monovalent salt (including $\mathrm{H}^{+}$) shown in Figure 1.2 as a sodium ion.

\subsubsection{The Gel-to-Fluid Phase Transition for Supported Lipids}

Lipid bilayers experience their major phase transition around physiological temperatures. Below the phase transition, the bilayer is in a quasicrystalline "gel" phase (also 
referred to as the $L_{\beta^{\prime}}$ phase). In this phase, the lateral diffusion constant of lipids can be measured by fluorescence recovery after photobleaching. For DMPC, the diffusion constant is about $10^{2}$ times slower than in the liquid crystal "fluid" phase $\left(L_{\alpha}\right)$, for which the lateral diffusion constant is on the order of $10^{-6}-10^{-7} \mathrm{~cm}^{2} \cdot \mathrm{s}^{-1}$, depending on temperature $[26,27]$. Structurally, the fluid phase has an about $8 \%$ larger area per lipid, characteristic among lipids of different tail length and charge [28].

The phase transition temperature depends most sensitively on the length of the aliphatic alkane chain with longer-tail lipids having higher transition temperatures. For the molecules studied in this work, both of which have two fully saturated carbon chains with 14 carbons per chain, the phase transition temperature for the vesicle geometry is about $23{ }^{\circ} \mathrm{C}$. For anionic lipid vesicles, the phase transition is also highly dependent on salt and lipid concentration due to the strong interaction between head groups which is less significant in neutral lipids [29].

However, the phase transition temperature for both molecules is shifted to higher temperature and broadened when the membrane is adsorbed on a substrate in air. Previous AFM work on supported bilayers suggests that the gel-fluid phase transition is extended across several degrees and includes a coexistence region wherein gel and fluid phases exist in domains [28,30-33].

As Chapter 2 will illustrate, Atomic Force Microscopy is a powerful tool for investigating the phase properties of supported lipids. The fluid phase, due to the disorder in the tails, is measurably thinner than the gel phase. Because we are comparing our experimental results with molecular dynamics simulations, performed for free (unsupported) bilayers in the fluid phase, AFM can be used to asses the extent to which the substrate perturbs the bilayer by measuring the deviation of the gel-to-fluid phase 
transition temperature from that of free vesicles.

\subsubsection{Properties of Anionic Lipid Bilayers}

In this work, the anionic lipid DMPG will be studied in addition to its neutral analog DMPC. The structural difference lies exclusively in the replacement of the terminal positively-charged choline group (in DMPC) with a neutral glycerol shown in Figure 1.2 .

Despite the minor structural difference between DMPC and DMPG, the anionic charge configuration significantly affects the phase behavior, stability, structure, and dynamics of the DMPG layer. For DMPG vesicles at low lipid concentrations, the gel and fluid phases exist alone with a transition temperature that increases from 23 to $41{ }^{\circ} \mathrm{C}$ going down from $1 \mu \mathrm{M}$ to $100 \mu \mathrm{M}$ concentration. At concentrations between $200 \mu \mathrm{M}$ and $800 \mathrm{mM}$, the gel and fluid phases coexist over a temperature range which can span up to $20^{\circ} \mathrm{C}[29]$.

At very low lipid concentrations, the DMPG bilayer can be neutralized by the small number of $\mathrm{H}$ ions in neutral water. The neutralization of the bilayer tends to shift the phase transition temperature upwards because the repulsive head group interaction is partly responsible for driving the membrane into the fluid phase. By the same mechanism, at higher lipid concentrations, the bilayer can be fully neutralized at low $\mathrm{pH}$ and stabilized in the gel phase.

The gel phase can be stabilized to higher temperatures with the introduction of $\mathrm{Ca}^{2+}$ ions [34]. It has been shown that $\mathrm{Mg}^{2+}, \mathrm{Ba}^{2+}$, and $\mathrm{Sr}^{2+}$ also cause the same effect. Furthermore, the presence of divalent ions is critical to adsorption of vesicles to surfaces [35]. 
At higher monovalent salt concentrations the phase transition tends to increase slightly [29]. The dependence of the transition temperature on both the lipid and ionic concentration results from a change in the bilayer charge state. Under conditions which leave the bilayer partially charged (lipid concentration high compared to ionic concentration, remembering the presence of $\mathrm{H}+$ ions from water), the gel-to-fluid transition temperature is shifted to lower temperature. This shifting is due to the repulsive interaction between the head groups which tends to encourage the fluid phase. On the other hand, the presence of monovalent salts can provide a screening effect, reducing the head group repulsion and stabilizing the gel phase.

The transition is driven by the melting of the alkane chains, which requires the bilayer area per lipid to increase to accommodate the flexible chains. A high surface charge tends to encourage head groups mobility due to electrostatic repulsions. The effect of divalent cation concentration is understood from the opposite perspective. Neighboring head groups tend to attract each other through the sharing of a divalent ion. In that case, more energy is required to increase the area per lipid, raising the gel-fluid phase transition temperature.

With regard to structure, the surface area per lipid is about $19 \%$ smaller for PG lipids compared to their PC counterparts due to the mediation of counterion bridges. The lateral diffusion of PG lipids in the bilayer is also slower for the same reason [36-38].

The magnitude of the membrane surface potential, describing the energy required to move a positive test charge nearer to the membrane, increases with higher ionic strength; furthermore, the surface charge density also decreases with increasing ionic strength [39]. For low ionic strengths, below about $15 \mathrm{mM}$, the membrane surface 
potential can be on the order of $-150 \mathrm{mV}$ (less than $-25 \mathrm{mV}$ may be considered "highly charged") [40]. A secondary effect is the formation of an electric double layer (multiple layers of positive and negative charges accumulating around the vesicle surface), which tends to prevent vesicle aggregation.

Because of the extended gel phase and very high surface potential (neighboring vesicles prefer to avoid each other), the vesicle structure for DMPG is extremely stable. As such, deposition of DMPG membranes on a substrate by vesicle fusion is difficult compared to the DMPC case. A novel method for preparing DMPG bilayers which overcomes these challenges is presented in Chapter 2.

Furthermore, because of the significant electrostatic potential extending away from the membrane surface, the orientation, dynamics, and freezing behavior of water molecules may also be different than for uncharged lipids. Under a modified GouyChapman-Stern model, used to model the electric double layer, the electrostatic potential decays exponentially as a function of the distance from the surface with a characteristic length equal to the Debye screening length [40]. The Debye length is a measure of the strength of the electrostatic interaction in a liquid compared to thermal energies. At physiological conditions, the Debye length is on the order of 1-2 nm, decreasing with increasing ionic strength. However, the Debye length is proportional to $T^{1 / 2}$ and can extend several $\mu \mathrm{m}$ from the surface at low temperatures in pure water. Thus, the strength of the electrostatic potential due to a charged surface can be significant at low temperatures.

To summarize, the phase diagram, stability, structure, and dynamics of the PG membrane differ from the PC bilayer. Fundamentally, the charge configuration of the head group strongly determines the distinctive behavior of PG bilayers. In the case of 
PC membranes, the alkane tails are primarily responsible for determining the phase transition temperature, fluidity, and stability; importantly, the head group charge is zero and these properties depend only weakly on ionic strength. For PG lipids, the ionic strength and charge configuration of the head group is of primary importance, thus adding another layer of complexity to the system.

Because of the addition of a significant electrostatic interaction for DMPG membranes, we posit that the interaction between the lipid head group and water molecules could be different than for the neutral DMPC system. We will investigate the influence, and difference between, the neutral and anionic head groups on water freezing using elastic neutron scattering..

\subsection{Neutron Scattering Background}

\subsubsection{Incoherent Neutron Scattering}

Scattering from a collection of atoms has the property that the measured intensity can arise both from the coherent addition of scattered waves but also from the sum

of individual incoherent scattering events. Incoherence arises from disorder in the system. For instance, a crystal may be heated such that the individual atoms can translate outside their crystalline atomic positions.

For neutron scattering, an additional type of incoherence is inherent in the measurement because the neutron scatters from the nucleus. The neutron scatters from the spin states of the nucleus which do not interact with their neighbors. Every isotope has a different configuration of spin states, which we can describe in terms of the average value (across all nuclei of a particular isotope in the sample) and the de- 
viation from this value [41]. Coherent scattering, which represents correlations across distant nuclei, can only arise from the average value of the spin states (more precisely, from the average interaction of the neutron with the spin states), whereas incoherent scattering can arise from any nucleus, but its intensity can only be related to the deviation of the spin states from their average value (otherwise, it would be part of the coherent scattering).

In short, coherent scattering is the sum of interactions of the neutron wave with the average scattering center whereas incoherent scattering is the sum of interactions of the neutron wave with individual nuclei. These quantities are clearly different because the spin of a nucleus is not correlated with its neighbors except at very low temperatures. Also, because the incoherent scattering cannot be correlated with its neighbor, it is by definition isotropic.

This distinction between incoherent and coherent scattering manifests itself in the scattering cross sections of isotopes. Each isotope has both a coherent and an incoherent cross section representing the average value and deviation from the average value of the strength of the nuclear interaction with the neutron, respectively. While it is theoretically possible to calculate the scattering cross section for any particular isotope, it is practically difficult and cross sections for elements heavier than helium are generally measured and tabulated instead.

A second inherent contribution to incoherent scattering is related to the natural isotopic concentrations of materials as different isotopes of the same element will contribute different scattering cross sections. Therefore, a common technique in neutron scattering is to isolate a particular isotope during sample production to achieve favorable cross sections [42]. 
There are two reasons why incoherent scattering is advantageous for studying water diffusion. First, as we have mentioned, incoherent scattering arises from the sum of interactions of the neutron wave with individual nuclei. As such, it can provide microscopic information such as the self-correlation function. The translational diffusion constant of water, which is defined as the proportionality between density gradient and water flux via Fick's law, is related to macroscopic quantities; however, incoherent neutron scattering can arrive at the same quantity through a microscopic measurement.

Second, a natural convenience is the anomalously large incoherent cross section of hydrogen which greatly increases the signal-to-noise ratio for any measurement interested in hydrogen (such as water) and increases scattered flux for an inherently flux-limited technique. A derivation of the cross-section of hydrogen is found in Appendix C.

\subsubsection{The Dynamic Structure Factor}

Neutron spectroscopy probes the dynamic structure factor

$$
\begin{aligned}
& S_{i n c}(\mathbf{Q}, \omega)=\frac{1}{2 \pi} \int_{-\infty}^{\infty} I_{i n c}(\mathbf{Q}, t) \exp (-i \omega t) d t \\
& I_{i n c}(\mathbf{Q}, t)=\frac{1}{N} \sum_{i=1}^{N} \exp \left[i \mathbf{Q} \cdot\left(\mathbf{R}_{i}(t)-\mathbf{R}_{i}(0)\right)\right] .
\end{aligned}
$$

The incoherent intermediate scattering function $I_{i n c}(\mathbf{Q}, t)$ here represents correlations between the same nucleus (labeled by $i$ which are positioned at $\mathbf{R}_{i}$ ) at different times separated by $t$ [41]. The summation indicates that we perform this calculation for every nucleus in the sample of which there are $N$. The dynamic structure 
factor $S_{i n c}(\mathbf{Q}, \omega)$ is the time Fourier transform of the intermediate scattering function, wherein we convert time to (angular) frequency, $\omega$. The intermediate scattering function itself is a space Fourier transform of the van Hove function

$$
\begin{gathered}
I(\mathbf{Q}, t)=\int G(\mathbf{r}, t) \exp [-i \mathbf{Q} \cdot \mathbf{r}] d \mathbf{r} \\
G(\mathbf{r}, t)=\frac{1}{N} \int\left\langle\rho\left(\mathbf{r}^{\prime}-\mathbf{r}, 0\right) \rho\left(\mathbf{r}^{\prime}, t\right)\right\rangle d \mathbf{r}^{\prime}
\end{gathered}
$$

In the above equation, $\rho(\mathbf{r}, t)$ is a particle density operator defined in such a way that $\int G(\mathbf{r}, t) d \mathbf{r}=N$, the total number of particles. In general, a scattering law for a particular type of motion can be developed by beginning with a real-space and time description of the motion expressed in terms of the van Hove function then transforming this function in space to the intermediate scattering function and again in time to the dynamic structure factor.

In the above expression for $I_{i n c}(\mathbf{Q}, t)$ and $S_{i n c}(\mathbf{Q}, \omega), \omega$ is the amount of energy gained or lost by the neutron according to $E=\hbar \omega$. The amount of energy gained or lost by the neutron is related to a time-scale $\Delta t$ by $\Delta E=h / \Delta t$. Usually, the convention is to plot the amount of energy gained or lost by the sample such that $\omega<0$ indicates neutron energy gain and $\omega>0$ indicates neutron energy loss. The phenomena discussed in this thesis are described by functions which are symmetric around $\omega=0$ such that this distinction will not be relevant.

The wave vector transfer, $\mathbf{Q}$, of the neutron defines the length scale of the experiment

$$
\mathbf{Q}=\mathbf{k}_{f}-\mathbf{k}_{i}
$$


where $\mathbf{k}_{f}$ and $\mathbf{k}_{i}$ are the outgoing and incoming neutron waves, respectively [41]. The wave-vector transfer is related to the momentum transfer of the neutron, $\Delta \mathbf{p}$, by $\mathbf{Q}=\Delta \mathbf{p} / \hbar$. In these experiments, the amount of energy transferred to or from a neutron is exceedingly small, on the order of tens or hundreds of $\mu \mathrm{eV}$ compared to the neutron's kinetic energy on the order of a few meV $[42,43]$. This amount of energy transfer is insufficient to change the wavelength of the neutron significantly. Therefore, when discussing the quantity of momentum transferred to or from the neutron, we are referring to its direction only; its magnitude remains unchanged. In other words, $k_{f}=k_{i}=k$ is a reasonable approximation that we will use. $\mathbf{Q}$ is related to the angle $2 \theta$ the scattered neutron makes with the incident beam

$$
Q=2 k \sin \theta
$$

The largest measurable $\mathbf{Q}$ occurs when $\mathbf{k}_{i}=-\mathbf{k}_{f}$ and is twice the incident wave vector. Positioning a detector at a particular angle with respect to the incident beam will correspond to a particular $\mathbf{Q}$ value. In general, $\mathbf{Q}$ is a three-dimensional vector; but in these experiments we are only concerned with the magnitude of $\mathbf{Q}$ because, as discussed in the previous section, incoherent scattering is isotropic. Due to $Q$ having units of inverse length, it can be more intuitive to consider the quantity $d=2 \pi / Q$ as a probing length. For our experiments, the accessible $Q$ range is about 0.3 to 1.9 $\AA^{-1}$, corresponding to distances between 3 and $20 \AA$.

We can view the intermediate scattering function from another perspective now. Because the quantity $\mathbf{Q} \cdot[\mathbf{R}(t)-\mathbf{R}(0)]$ appears in the exponential, values of $\mathbf{Q} \cdot[\mathbf{R}(t)-$ $\mathbf{R}(0)$ ] much larger than $2 \pi$ result in rapid oscillations in time, which will average to zero when integrated in Eq. 1.1. Thus, for a given $\mathbf{Q}$ value, only $\mathbf{R}(t)-\mathbf{R}(0)$ values 
such that $\mathbf{Q} \cdot[\mathbf{R}(t)-\mathbf{R}(0)]$ is on the order of $2 \pi$ will be contribute to $S_{\text {inc }}(\mathbf{Q}, \omega)$. This discussion is another way to visualize the accessible length scale of our experiments.

Backscattering spectroscopy has poor $Q$-resolution relative to triple-axis inelastic spectroscopy or diffraction techniques. To achieve the minimum spread in wavelength, the neutrons must backscatter from an analyzing crystal which rejects a large fraction of the beam scattered from the sample [44]. In order to maximize neutron flux, detectors are designed to cover a larger solid angle at the price of having fewer detectors and hence coarser $Q$ resolution. Physical processes that can be probed by backscattering spectroscopy have characteristic $Q$-dependence which is usually smooth, unlike Bragg peaks in elastic diffraction patterns. The poor $Q$-resolution of backscattering spectroscopy is not a limitation for the investigations described here. The $Q$-dependence of a measured scattering process can be an important clue into its origin as we will see in the following sections.

\subsubsection{Elastic Neutron Scattering}

One type of measurement that will be discussed in Chapters 3 and 4 is a so-called elastic intensity scan. These measurements are a special case called elastic scattering wherein neutrons transfer less energy than the minimum detectable amount. The elastic scans can be used to measure the quantity of frozen water in our membrane samples as a function of temperature and can provide clues about which temperatures to investigate further with full quasielastic spectra.

In practice, it is not possible to measure arbitrarily small energy transfers. The limit on the smallest measurable energy transfer, $\Delta E_{\min }$ is the instrumental resolution, and it defines the maximum time scale of the motion that can be investigated: 


$$
\Delta E_{\min }=\frac{h}{t_{\max }}
$$

where $t_{\max }$ is the time scale of the slowest motion that can be detected. Suppose, as an example, that we are interested in following the motion of a single water molecule as it diffuses. We can describe the position of the water molecule (or more precisely, the nucleus of a hydrogen atom in a water molecule) by the vector $\mathbf{R}(t)$ as it appears in the intermediate scattering function where we have dropped the subscript $i$ because we are interested in a system of only one nucleus. Let the nucleus begin at $\mathbf{R}(t=0)$. At some later time, the nucleus is at $\mathbf{R}(\Delta t)$. Consider a situation in which the nucleus is stationary for the time $\Delta t$. Such a scenario could be that the water molecule is locked in a crystal lattice. The intermediate scattering function in this case is simply unity, as $\mathbf{R}(\Delta t)-\mathbf{R}(0)=0$ for a stationary nucleus. To evaluate the dynamic structure factor, we have

$$
\begin{aligned}
S_{i n c}(\mathbf{Q}, \omega) & =\frac{1}{2 \pi} \int_{-\infty}^{\infty} \exp (-i \omega t) I_{i n c}(\mathbf{Q}, t) d t \\
& =\frac{1}{2 \pi} \int_{-\infty}^{\infty} \exp (-i \omega t) d t \\
& =\delta(\omega)
\end{aligned}
$$

where $\delta(\omega)$ is the Dirac delta function. In other words, if the nucleus is stationary for a long time, the dynamic structure factor is a delta function centered at zero energy transfer. Nuclei that are stationary for times longer than $t_{\max }$ will scatter elastically. It is not a requirement that a nucleus be locked in a crystal structure 
(such as hexagonal bulk ice) to scatter elastically; instead, it must only be stationary on a particular time scale. In Section 1.3 we will give examples of the case where the nucleus is not stationary on the time-scale $t_{\max }$.

\subsubsection{Energy Resolution for Backscattering Spectrometers}

The maximum time scale that can be measured is related to the minimum energy transfer that can be detected, as described in the previous section. Backscattering spectroscopy can measure extremely small energy transfers. In this section, we will discuss the details of the backscattering spectrometers HFBS and BASIS. These instruments are notable because their high energy resolution is coupled with high neutron throughput. Both HFBS and BASIS provide exceptional energy resolution by exploiting Bragg's law for the monochromation of neutrons $[43,44]$

$$
n \lambda=2 d \sin \theta .
$$

Here, $d$ is a lattice spacing in a crystal, $\theta$ is the angle of the incident beam measured with respect to a lattice plane, and $\lambda$ is the incident wavelength of the neutron. We can assume for this discussion that $n$, the order of the diffraction, is 1 . The spread in wavelength of the diffracted beam is found by differentiation of Eq. 1.11. For high energy resolution, we wish to minimize $\delta \lambda$

$$
\frac{\delta \lambda}{\lambda}=\frac{\delta d}{d}+\frac{\delta \theta}{\tan \theta} .
$$

It is clear that the minimum of this expression is achieved for $\theta=90^{\circ}$, which eliminates the second term. The first term is then the determining factor in the wavelength 
spread and hence the energy resolution. The quantity $\delta d$ represents a spread in $d$ spacings due to any inherent imperfections in the crystal and the Darwin width of the reflection. The Darwin width is a consequence of a subtle dynamical effect that limits the minimum width of a Bragg reflection in reciprocal space. Neutrons scattered from a crystal at or close to $90^{\circ}$ will have the minimum spread in wavelength. This geometry is called backscattering as the outgoing neutrons will retrace their paths after scattering $\left(2 \theta=180^{\circ}\right)$.

In practice, the full-width-at-half-maximum (FWHM) of the energy resolution of HFBS and BASIS is $\pm 1 \mu \mathrm{eV}$ and $\pm 3.5 \mu \mathrm{eV}$, respectively, and is roughly Gaussian in shape [44]. The 1 and $3.5 \mu \mathrm{eV}$ energy resolutions correspond to maximum time-scales of about 4 ns and 1 ns respectively. Many important processes in fluids and biology occur on time-scales of about 10 ps to $1 \mathrm{~ns}[45,46]$. Backscattering spectroscopy is one of the few neutron techniques that can provide the sensitivity to probe these time-scales. For comparison, the average residence time of a water molecule tightly bound to the surface of a biological molecule, such as a lipid head group or protein, is on the order of several hundred ps, which can be inferred directly from the width of the quasielastic broadening by measuring neutron counts as a function of $\Delta E$ and fitting to a Lorentzian function [42]. Such a motion would not be accessible with other inelastic neutron scattering techniques.

In order to observe translational diffusion of supercooled water, high energy resolution is required. At $260 \mathrm{~K}$, the diffusion constant of bulk supercooled water is $4.29 \times 10^{-6} \mathrm{~cm}^{2} \cdot \mathrm{s}^{-1}$, or $57 \mu \mathrm{eV} \AA^{2}$ in convenient scattering units [47]. At a typical $Q$ value of $0.5 \AA^{-1}$, the width of the quasielastic scattering is $14 \mu \mathrm{eV}$. As such, in order to explore the supercooled regime (discussed in Chapter 3), we must make use 
of the exquisite energy resolution provided by backscattering spectrometry. Traditional inelastic neutron scattering methods cannot approach the energy resolution of backscattering spectrometry.

Narrower energy resolution comes at the price of neutron throughput. The HFBS instrument can probe time-scales three times longer than BASIS; thus, it is ideal for elastic scans. However, in order to measure full quasielastic spectra, which are necessary to extract dynamic properties such as the diffusion constant of water, the neutron throughput of BASIS is necessary.

\subsection{Modeling Water Diffusion}

\subsubsection{Einstein Model of Brownian Diffusion}

As an example of how physical properties can be extracted by measuring the incoherent neutron dynamic structure factor, we consider the case of translational water diffusion. A simple model of water diffusion can be illustrated in terms of a random walk as suggested by Einstein in 1905 [41]. In this model, water simply translates freely until colliding with another particle at which point it changes its direction ran-

domly and independently of its previous trajectory. It is possible to show that the van Hove function under these assumptions obeys

$$
\tau \frac{\partial G(\mathbf{r}, t)}{\partial t}=\frac{\left\langle l^{2}\right\rangle}{6} \nabla^{2} G(\mathbf{r}, t) .
$$

Here, $G(\mathbf{r}, t)$ is the probability density of finding a water molecule at a position $\mathbf{r}$ at time $t, \tau$ is the average time between collisions, and $\left\langle l^{2}\right\rangle$ is the mean square displacement after a time $\tau$. This equation is a particular expression of Fick's law, 
which in general relates the concentration gradient of a fluid to its flux. We demand the initial condition that $G(\mathbf{r}, 0)=\delta(\mathbf{r})$; that is, a water molecule begins at the origin at $t=0$. Also, we require that $\int G(\mathbf{r}, t) d \mathbf{r}=1$. A solution to Eq. (1.13) is

$$
G(\mathbf{r}, t)=(4 \pi D t)^{-3 / 2} \exp \left(-r^{2} / 4 D t\right) .
$$

$D$ is called a diffusion constant and can be related to the microscopic variables $\tau$ and $\left\langle l^{2}\right\rangle$ such that $D=\left\langle l^{2}\right\rangle / 6 \tau$. By taking two Fourier transforms, $G(\mathbf{r}, t)$ can be used to calculate the dynamic structure factor measured in a neutron scattering experiment. The first transformation from real space to reciprocal space is

$$
\begin{aligned}
I_{i n c}(\mathbf{Q}, t) & =\int_{0}^{\infty} G(\mathbf{r}, t) \exp (-i \mathbf{Q} \cdot \mathbf{r}) d \mathbf{r} \\
& =\exp \left(-D Q^{2} t\right) .
\end{aligned}
$$

To obtain the dynamic structure factor, we transform this result in time:

$$
\begin{gathered}
S_{i n c}(\mathbf{Q}, \omega)=\frac{1}{2 \pi} \int_{-\infty}^{\infty} I_{i n c}(\mathbf{Q}, t) \exp (-i \omega t) d t \\
S_{i n c}(Q, \omega)=\frac{1}{2 \pi} \int_{-\infty}^{\infty} \exp \left(-D Q^{2} t\right) \exp (-i \omega t) d t \\
S_{i n c}(Q, \omega)=\frac{1}{\pi} \frac{D Q^{2}}{\omega^{2}+\left(D Q^{2}\right)^{2}} .
\end{gathered}
$$

The dynamic structure factor is then a Lorentzian with $\Gamma=D Q^{2}$, where $\Gamma$ is the half-width-at-half-maximum (HWHM). Furthermore, the intensity of the Lorentzian 
function, described by the integral of $S_{\text {inc }}(Q, \omega)$ over all $\omega$, is constant as a function of $Q$. This dynamic structure factor describes a quasielastic shape: the scattering is broadened in some way (here, as a Lorentzian) and centered at zero energy transfer $(\omega=0)$.

\subsubsection{Jump Diffusion Model}

The model presented above for the diffusion of water above is not unique, however. Another frequently employed model is a jump-diffusion model, or the Chudley-Elliott model, in which individual water molecules move ballistically for a short amount of time inside a "cage" formed by the neighboring water molecules that they cannot penetrate on short time scales $[47,48]$. On longer time scales, water molecules are capable of "jumping" to another site, allowing them to move in a new cage formed by surrounding molecules. The jump diffusion model shows a characteristic flattening of the quasielastic width at higher $Q$, characteristic of motion of water molecules confined in a fixed volume, as seen in Figure 1.3 [47].

Following the development of Hall and Ross [49], in which the probability of a jump of a particular length $\rho(l)$ is given by an isotropic distribution of the form

$$
\rho(l)=\frac{2 l^{2}}{l_{0}^{3} \sqrt{2 \pi}} \exp \left(-l^{2} / 2 l_{0}^{2}\right),
$$

which has the properties

$$
\int_{0}^{\infty} \rho(l) d l=1
$$




$$
\left\langle l^{2}\right\rangle=\int_{0}^{\infty} l^{2} \rho(l) d l=3 l_{0}^{2} .
$$

It is straightforward to derive the dynamic structure factor for this model [49].

$$
\begin{gathered}
S(Q, \omega)=\frac{1}{\pi} \frac{\Gamma_{J}(Q) \tau}{\left[\Gamma_{J}(Q)\right]^{2}+\omega^{2} \tau^{2}} \\
\Gamma_{J}=\frac{1}{\tau}\left[1-\exp \left(-\frac{Q^{2} l_{0}^{2}}{2}\right)\right] .
\end{gathered}
$$

As found earlier for the Einstein model, the quasielastic width of the jump diffusion model $\Gamma_{J}$ varies as $D Q^{2}$ as $Q \rightarrow 0$. Note that for this work the high $Q$ value of $\Gamma_{J}$ for this model approaches a constant value, $1 / \tau$. More recently, models have been introduced that include coupling of rotational and translational motion, leading to complicated high $Q$ behavior [50-53]. Modeling water in confinement presents additional challenges such as the non-isotropic distribution of jump lengths [54-56]. However, all of these models of water translational diffusion predict that $\Gamma$ varies as $Q^{2}$ at low $Q$. Thus the determination of the diffusion constant is model independent.

\subsubsection{Neutron Scattering from Supercooled Water}

The purpose of discussing the Einstein and Chudley-Elliott models is to understand the maximum value of $Q$ for which the $\Gamma=D Q^{2}$ approach to determining the diffusion constant is valid. In Chapter 3, the diffusion constant for supercooled water in the vicinity of DMPC membranes will be discussed and analyzed. The value of $D$ will be determined by restricting the upper limit on $Q$ to $0.9 \AA^{-1}$.

Furthermore, the QENS results for DMPC membranes reveal a second type of water, distinct from bulk supercooled water that is only accessible in the low-temperature 
regime by freezing out the bulk-like contribution. An careful determination of the diffusion constant is necessary to make this identification.

In a pair of seminal papers, Teixeira et al. and Chen et al. measured the quasielastic scattering from supercooled bulk water in $300 \mu \mathrm{m}$ diameter Pyrex capillaries [47,57]. These measurements were performed on several instruments, the most sensitive of which had an energy resolution of $\pm 35 \mu \mathrm{eV}$. Their results, shown in Figure 1.3, clearly demonstrate a significant leveling off of the quasielastic width at high $Q$ even at $-5^{\circ} \mathrm{C}$. As the temperature is lowered, the linear regime at low $Q$ includes only a few points with $Q<0.8 \AA^{-1}$. Therefore, it is clear that determining the diffusion constant by measuring the slope of the quasielastic scattering as a function of $Q$ is only valid for very low $Q$ at low temperatures. 


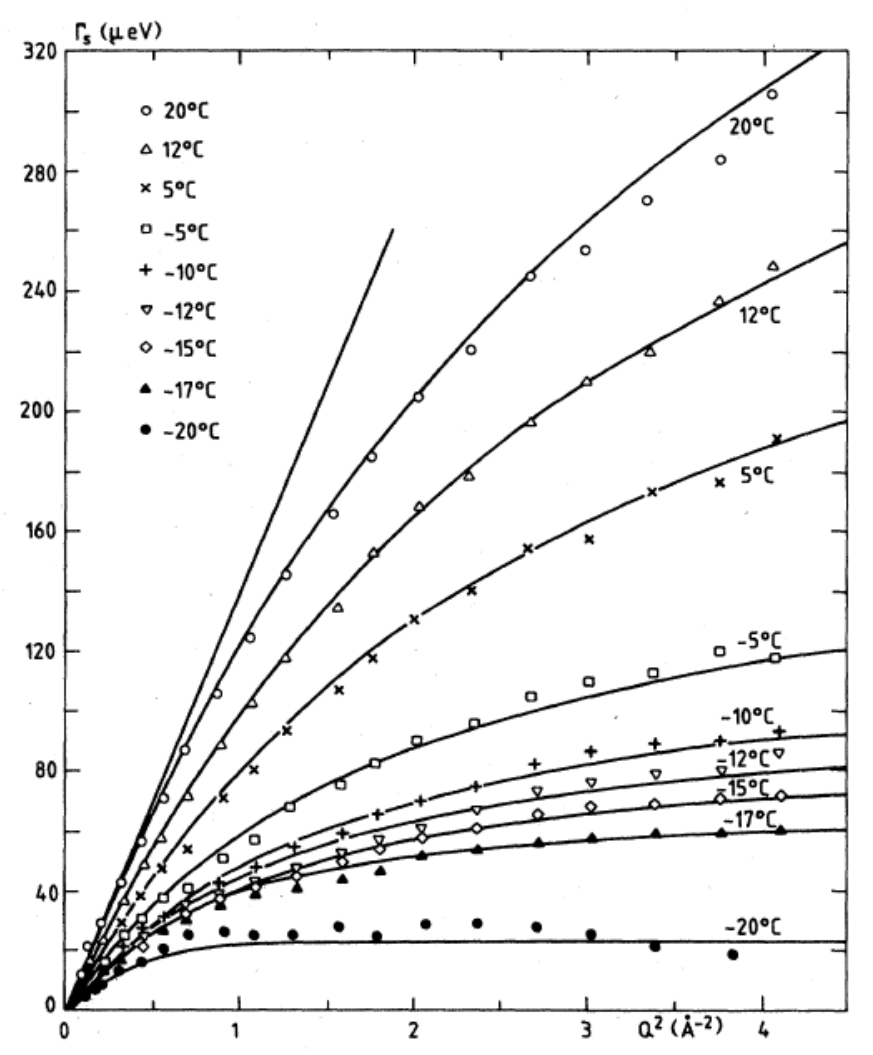

Figure 1.3: The quasielastic width $\Gamma$ of supercooled water as determined by Teixeira et al. [47]. The black lines represent the best fit to the jump diffusion model. The data clearly show a flattening of the quasielastic width at higher $Q$ values. Only at low $Q$ does the quasielastic width have a linear behavior. The $Q$ value above which the linear approximation fails decreases at low temperatures. 


\subsection{Quasielastic Scattering from Lipid Membranes}

Quasielastic neutron scattering has been applied to lipid membranes to study motion of both lipid and water molecules. In this section, we review the literature reports of previous QENS measurements. The work in this thesis represents the first study of water freezing and dynamics in the vicinity of single-supported lipids of both PC and PG head group types. A single report exists of temperature-dependent elastic neutron scattering from single-supported DMPC membranes at low temperatures [58]. The topic of molecular motions of lipid membranes at physiological temperatures has received more attention.

\subsubsection{Lipid Motions}

In an early work by Pfeiffer et al., oriented multilayers $(25 \mu \mathrm{m}$ thick in total) of protonated and tail-deuterated DPPC (an analogue of DMPC with 16 carbon tails) were used to study the various motions of lipid molecules in both the fluid and gel phases [59]. Data were collected below and above the phase transition temperature $\left(41.3{ }^{\circ} \mathrm{C}\right.$ for DPPC). By deuterating both the lipid tails and the solvent, it was possible to probe selectively the motion of the remaining hydrogens in the PC head group; furthermore, the membrane stack could be rotated with respect to the incident beam to probe motions in and out of plane. Four types of motions were identified for the lipids themselves: first, a bounded, conformational chain-defect motion (100 ps); second, a rotational motion of the lipid tails about their long axis (ns); third, an in-plane diffusion of the lipids (100 ns); and fourth, a rotational motion of the head group (100 ns).

The lateral diffusion of the lipids themselves, both in and out of the plane, could 
not be detected in the gel phase. Interestingly, the motion of the lipid chains appears in the gel phase as well as the fluid phase. Evidence from NMR results suggest that this chain defect motion continues well below $0{ }^{\circ} \mathrm{C}[60]$.

Lateral diffusion of lipids has subsequently been measured with QENS using highenergy-resolution spectroscopy [61,62]. The lateral diffusion of DMPC was measured by observing a quasielastic broadening on the order of $10 \mu \mathrm{eV}$, corresponding to a diffusion constant of $6 \times 10^{-8} \mathrm{~cm}^{2} \cdot \mathrm{s}^{-1}$ at $303 \mathrm{~K}$ (in the fluid phase), or about $10^{3}$ times slower than water motion at the same temperature. In this case, a simple Fickian diffusive motion with linear $Q^{2}$ dependence as discussed in Section 1.3 was applied. The diffusion constant of lipids measured in this way is commensurate with literature reports using other techniques further supporting the feasibility of these types of measurements.

\subsubsection{Water Dynamics near Lipid Membranes}

A combined QENS and NMR study as a function of hydration indicated two motions of water molecules at high temperature $\left(55^{\circ} \mathrm{C}\right.$, in the fluid phase for DPPC) [63]. Multilayer stacks of $20 \mu \mathrm{m}$ total thickness were used. At low hydration, corresponding to just three to four water molecules per lipid, a rotational motion of water molecules in the vicinity of the head group with a time-scale of 60 ps was observed. At higher hydration, a translational diffusion component appears which agrees down to $2{ }^{\circ} \mathrm{C}$ with the model of Teixeria et al. discussed in Section 1.3.2 [47]. A "slow" (longer than 1 ns) motion in which water molecules move between the head group region and the bulk is also measured in the higher hydration sample. In Chapter 3, we will invoke a similar argument to explain the comparably slower dynamics of a component 
attributed to motion of head group-bound water molecules in a low-hydration sample.

Swenson et al. investigated dynamics of water, lipid chains, and lipid head groups with quasielastic neutron scattering [45]. The authors made use of selective deuteration to separate the dynamics and probed motions parallel and perpendicular to the bilayer (in this case, DMPC) by using two different sample orientations of lipid stacks consisting of thousands of membrane layers. At low temperatures (below 290 $\mathrm{K}$ to as low as $260 \mathrm{~K}$ ), the authors identified a rotational water motion. Near 290 $\mathrm{K}$, close to the gel-to-fluid phase transition, the water dynamics appeared to exhibit a translational character which was modeled with a jump diffusion model. Interestingly, conformational changes of the lipid tails could also be identified as low as 120 K. The authors did not identify translational water motion below $290 \mathrm{~K}$ due to the limited energy resolution of the instrument.

On the other hand, QENS measurements on bacteriorhodopsin (BR), an integral membrane proton pump protein which forms a two-dimensional crystal structure known as "purple membrane", have identified translational water dynamics at lower temperatures [64]. In stacks of thousands of these membranes, it has been found that interlayer water diffusion can be slower by as much as a factor of five and the rotational component slower by a factor of six compared to comparable bulk water at room temperature $(293 \mathrm{~K})$. In these experiments, protein layers are separated by about $60 \AA$ (amounting to about 650 water molecules per protein with a $12 \AA$ water layer between membrane layers).

The authors also found that by cooling their samples, the inter-layer distance could be decreased (measured by neutron diffraction). The exact temperature-dependence of this behavior was strongly influenced by total water content. A dehydration mecha- 
nism whereby water leaves the inter-layer region was proposed. Importantly, a strong supercooling effect of the remaining inter-layer water was observed, with complete freezing achieved at $265 \mathrm{~K}$ in higher hydration samples and as low as $255 \mathrm{~K}$ for lower hydration samples. However, the amount of water remaining between membrane layers at low temperature was on the order of two monolayers commensurate with the amount of "unfreezable" water measured by NMR [65]. It is possible that a similar dehydration mechanism is responsible for the freezing of bulk-like water in our membrane samples which appears to be suppressed to $265 \mathrm{~K}$.

But most importantly, the authors identified two quasielastic scattering components with temperature-independent widths (a "slow" component with a time scale $5.5 \mathrm{ps}$ and a "fast" component with a $0.5 \mathrm{ps}$ time scale) [64]. Both components appeared around $200 \mathrm{~K}$ where they were identified with stochastic motion. At $265 \mathrm{~K}$, the intensity of both quasielastic components demonstrates a change in temperature dependence which the authors identify as a transition to an unfrozen state. As with Swenson et al., the measurements were limited due to the $100 \mu \mathrm{eV}$ FWHM energy resolution of the instrument. Nevertheless, quasielastic scattering could be identified at temperatures below the freezing point of bulk water. The remaining unfrozen water still contributes to the quasielastic signal.

Both this measurement on purple membrane and a similar QENS measurement involving flash cooling the same system resulted in strong hysteresis between cooling and heating $[66,67]$. While water was expelled from the interlayer region on cooling, it does not return until the system is returned to high temperature. 


\section{Chapter 2}

\section{Atomic Force Microscopy on Lipid}

\section{Bilayers}

In this Chapter, three major topics will be discussed. First, deposition of DMPC bilayers of sufficiently high-quality for quasielastic neutron scattering measurements will be described using methods previously refined and presented [68]. Second, the protocol for preparing high-quality DMPG bilayers using novel methods will be outlined. Third, the strength of the interaction of the substrate with the bilayer will be indirectly measured by following the gel-to-fluid phase transition temperature for both membranes. This characterization is important for making comparisons to molecular dynamics simulations which are performed on free bilayers.

\subsection{Overview of the AFM Technique}

Atomic Force Microscopy is a technique used to study the topology of nanoscale structures with molecular resolution. In particular, the accessible length scales are 
commensurate with the size of proteins or other biologically relevant molecules. The AFM technique is highly versatile. It can be applied in both liquid and air environments and at a range of temperatures relevant to physiological conditions.

The basic operating principle of AFM is to interrogate the surface with an AFM tip, typically silicon nitride, which is engineered to possess an extremely small contact area. A routine application would involve a tip whose apex could be described by a hemisphere with a radius on the order of $10 \mathrm{~nm}$. The tip is brought in contact with the surface where it is attracted via long-range electrostatic and van der Waals forces. Changes in the topology of the surface cause the tip to be deflected in the $z$-direction, defined to be normal to the planar sample surface. The deflection is measured by a light beam which reflects from the back of the cantilever upon which the tip is affixed.

Two common modes of AFM operation are contact mode in which the tip-sample force is maintained constant and a dynamic "tapping" mode. In tapping mode, the tip is vibrated at the cantilever's resonant frequency; and the amplitude of the tip oscillation is held constant. A feedback mechanism translates the sample position in the $z$-direction to maintain constant force or amplitude as the sample is moved laterally by means of a piezoelectric stage. In this way, a three-dimensional image is created that maps the topology of the sample surface to the lateral $x$ and $y$ position.

For a basic schematic of the original technique, see Binning et al. [69]. A more comprehensive review of the application of AFM to biological systems can be found in Jalili and Laxminarayana [70] and a modern review in Müller and Dufrêne [71]. 


\subsection{Sample Preparation for DMPC Layers}

Silicon wafers provided the substrate for all samples unless otherwise noted. The circular wafers are double-side polished, $300 \mu \mathrm{m}$ thick, and $5.08 \mathrm{~cm}$ in diameter. Wafers are cut into squares (approximately $1 \times 1 \mathrm{~cm}$ ) prior to cleaning. They are cleaned using a "Piranha" solution composed of $\mathrm{H}_{2} \mathrm{SO}_{4}: \mathrm{H}_{2} \mathrm{O}_{2}$ in a 3:1 ratio designed to remove organic materials from the surface. Wafers are submerged in the Piranha solution for approximately $1 \mathrm{hr}$ at $110^{\circ} \mathrm{C}$, being flipped over once after 30 minutes. The wafers are then vigorously rinsed with high-purity water. Clean wafers are hydrophilic (water neither beads nor runs from the surface). Wafers that remain partially hydrophobic after this treatment (indicating incomplete surface cleaning) may require $10 \mathrm{~min}$ in a deionized water ultrasonic bath, designed to remove non-organic material from the surface, before a second bath in the Piranha solution.

DMPC $\left(\mathrm{C}_{36} \mathrm{H}_{72} \mathrm{NO}_{8} \mathrm{P}\right)$ is received in dry powder form from Avanti. ${ }^{1}$ The powder is then suspended in a buffer solution containing $100 \mathrm{mM} \mathrm{KCl}, 5 \mathrm{mM} \mathrm{MgCl}_{2}$, and 5 mM HEPES $\left(\mathrm{C}_{8} \mathrm{H}_{18} \mathrm{~N}_{2} \mathrm{O}_{4} \mathrm{~S}\right.$, a zwitterionic buffer material) in $18.2 \mathrm{M} \Omega$ water. DMPC powder is added to the solution at a concentration of $1.5 \mathrm{mg} / \mathrm{ml}$ and sonicated for $72 \mathrm{~h}$ at $30-50{ }^{\circ} \mathrm{C}$ or until the solution does not contain macroscopic clumps of material. This step is necessary to disperse and hydrate any DMPC powder clumps. The resultant solution contains multilamellar vesicles of various sizes as indicated by dynamic light scattering. At this point, the solution may be a milky-blue color, becoming more opaque at higher concentrations.

The solution is diluted in buffer to the desired concentration and clean silicon wafers are introduced into the solution at $65{ }^{\circ} \mathrm{C}$ and incubated for $1 \mathrm{~h}$. During the

\footnotetext{
${ }^{1}$ Avanti Polar Lipids, Alabaster, AL, USA. $\geq 99 \%$ purity
} 
incubation time, the vesicles spontaneously adsorb to the surface, fuse with neighboring vesicles, and rupture, resulting in layers of supported lipids [68]. This process is referred to as vesicle fusion. After $1 \mathrm{~h}$, the wafers are removed and appear macroscopically hydrophobic relative to the clean wafers with water running from the surface.

\subsection{Controlling DMPC Bilayer Coverage}

The key parameters in the vesicle fusion process for DMPC bilayers are the incubation time and the lipid concentration. Lower values of either of these parameters results in lower bilayer coverage. For simplicity, all DMPC samples are produced with one hour incubation time, a value that was found to produce high-quality bilayers at higher concentrations reliably. However, for experiments which require sub-bilayer coverage, a relationship between lipid concentration in the buffer solution and bilayer coverage is desired. By systematically varying the lipid concentration with all other parameters of the fusion process held constant, we can develop a map between concentration and coverage by characterizing with AFM.

Figure 2.1 shows a series of AFM images, collected in tapping mode, from DMPC layers produced by vesicle fusion using solutions of different concentration of DMPC material. Below $2 \mu \mathrm{g} / \mathrm{ml}$, no significant features are noted. At $2 \mu \mathrm{g} / \mathrm{ml}$ [panel (a)], vesicles on the order of 1-200 $\mathrm{nm}$ diameter are visible; however, the larger vesicles do not rupture into planar bilayers at this low concentration. Smaller features on the order of 50-100 nm are also present and may be individual micelles. Panel (b), at 15 $\mu \mathrm{g} / \mathrm{ml}$, shows the first evidence of planar bilayer formation. The circular features are flat and approximately $5.9 \mathrm{~nm}$ in height. This step height is consistent with literature

reports of gel-phase bilayer thicknesses when measured in air on glass substrates [8]. 


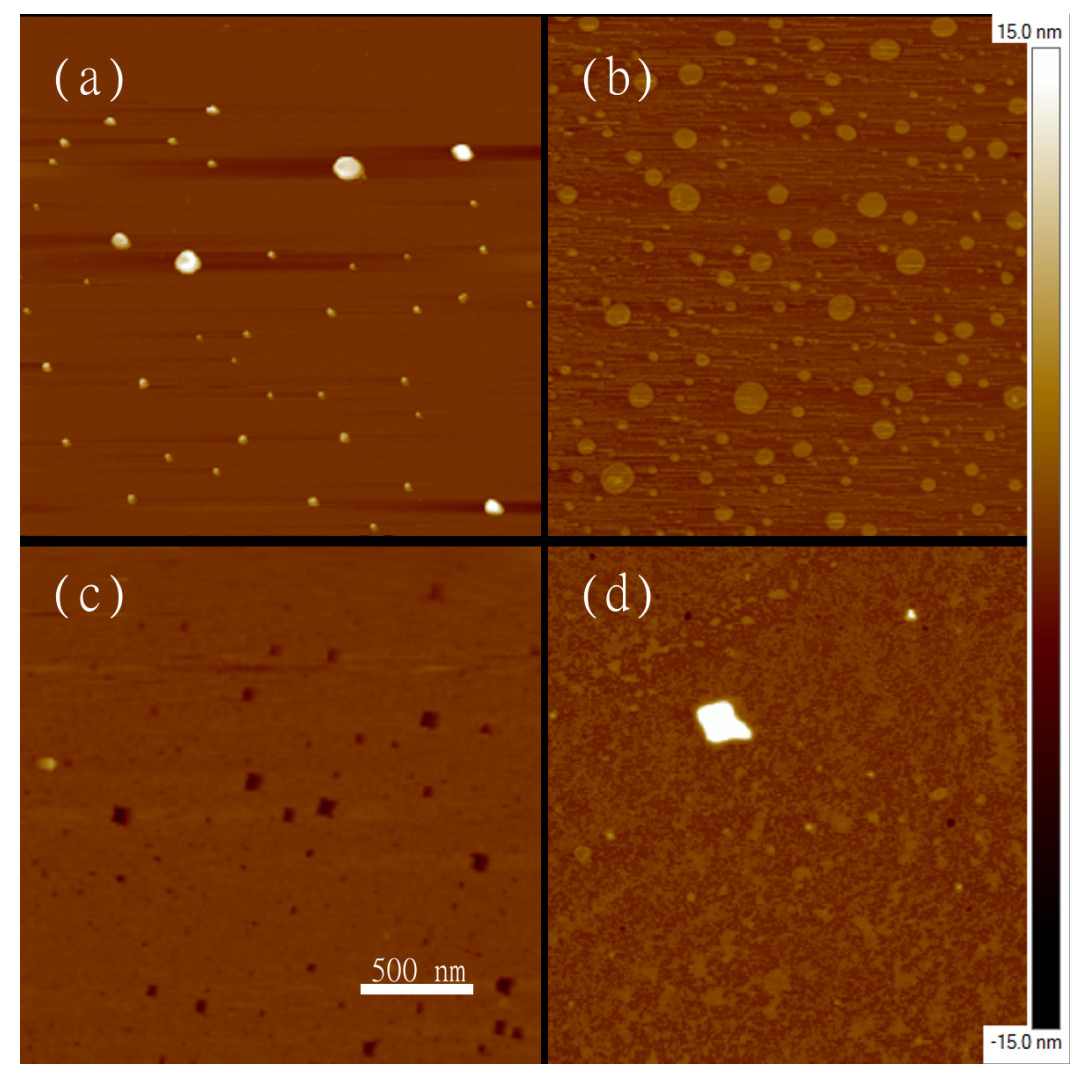

Figure 2.1: Bilayer membrane coverage as a function of lipid concentration as determined by AFM in the tapping mode. Typical AFM images of samples produced by incubating in a solution of (a) $2 \mu \mathrm{g} / \mathrm{ml}$; (b) $15 \mu \mathrm{g} / \mathrm{ml}$; (c) $30 \mu \mathrm{g} / \mathrm{ml}$; and (d) $60 \mu \mathrm{g} / \mathrm{ml}$ for $1 \mathrm{~h}$ at ${ }^{\circ} \mathrm{C}$. In panel (a), at low concentration, vesicles adsorb but do not rupture. Vesicles rupture into bilayers as the concentration is increased [panel (b)]. Bilayer coverage increases until nearly full layers are observed [panel (c)]. At high concentration [panel (d)], full bilayers are observed. Additional material forms aggregations (large feature in panel $(\mathrm{d})$ ) which can be removed by rinsing. Full bilayer coverage is only achieved with $\geq 30 \mu \mathrm{g} / \mathrm{ml}$. Each image is $2.5 \mu \mathrm{m} \times 2.5 \mu \mathrm{m}$. Vertical scale is 15 nm. 
However, it is $25 \%$ larger than on mica surfaces [72]. At $30 \mu \mathrm{g} / \mathrm{ml}$ in panel (c), the surface is almost completely covered with a single bilayer; in this image, the darker areas represent the uncovered silicon substrate which is measured to be at a depth of $5.9 \mathrm{~nm}$. Finally, for a $60 \mu \mathrm{g} / \mathrm{ml}$ lipid concentration [panel (d)], the surface is covered completely with a single bilayer and a partial second layer is seen to be formed. In addition, larger aggregations are visible at this concentration.

Quantifying the bilayer coverage is done via a histogram analysis. Every pixel is assigned a depth, defined as the vertical distance below the highest height in the image. The pixels are counted and binned according to their depth. This procedure provides a direct way of calculating the fraction of pixels below or above a particular threshold height. For example, in Figure 2.2, the original image on the left is binned such that any pixels representing an overlaying bilayer are shaded blue on the right. The plot below the image shows the fraction of pixels within the dashed rectangle with a depth below the value on the abscissa. In this particular image, the cursor is placed at $14 \mathrm{~nm}$ depth; all pixels with depth less than $14 \mathrm{~nm}$ (representing bilayer) are colored blue. Approximately $65 \%$ of pixels meet this criteria; so, we conclude that $65 \%$ of the area inside the dashed rectangle is covered in bilayer.

This procedure cannot account for multiple layer formation such as the feature inside the red circle in Figure 2.3. To include these features, the depth threshold is decreased to include only second layers; then, their area is added directly to the total number of bilayers. In this way, it is possible to characterize multilayer formation. By repeating this process over many images, the bilayer coverage as a function of lipid concentration is determined as shown in Figure 2.3.

The bilayer coverage is approximately linear in lipid concentration for low con- 


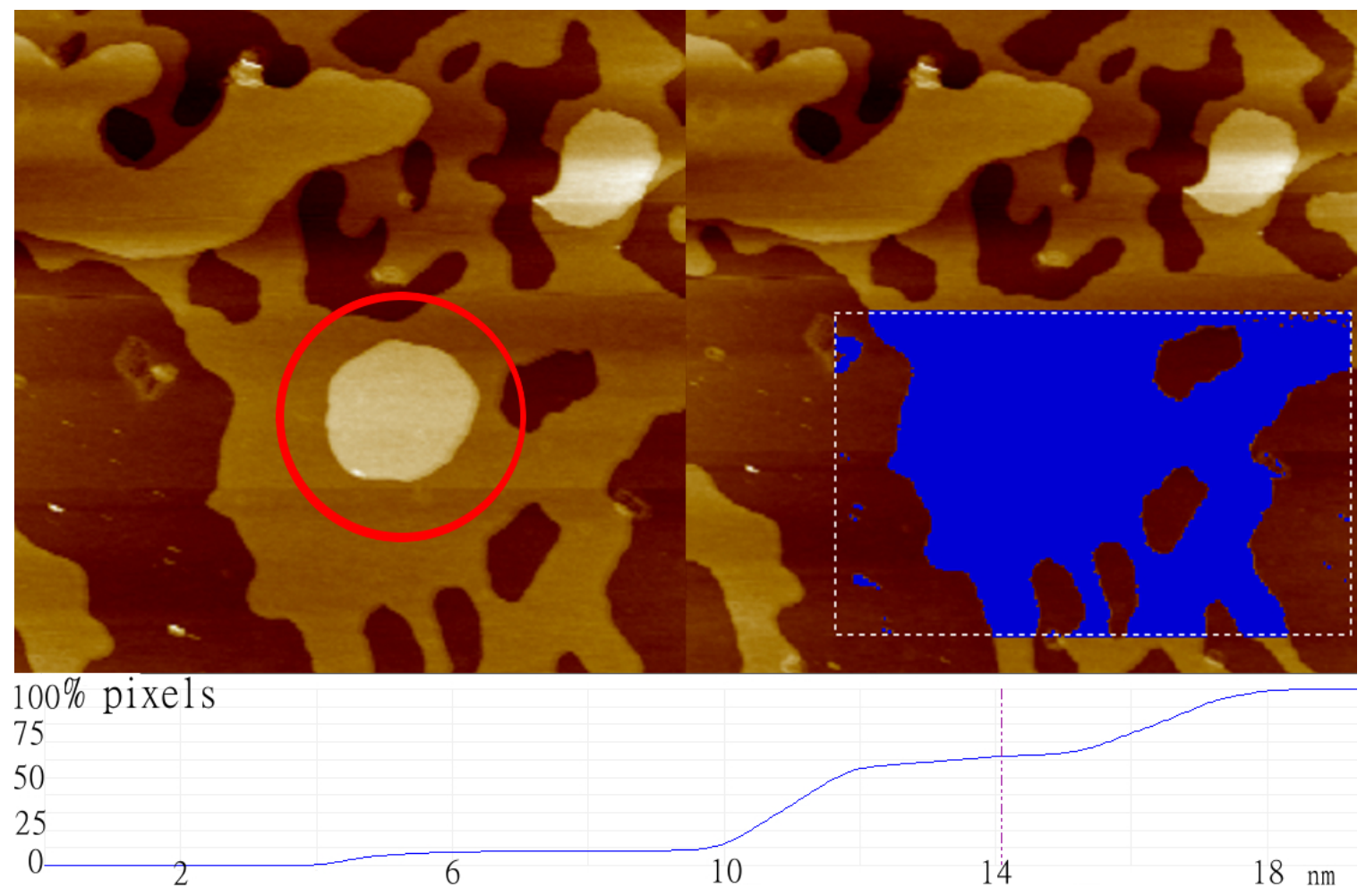

Figure 2.2: Example of the histogram analysis for obtaining bilayer coverage. Each pixel in the $512 \times 512$ pixel image is assigned a depth. Depth is defined as the difference between the highest pixel in the region and the height at the pixel. A region of interest can be selected as indicated by the rectangular box. The fraction of pixels below a given depth is shown in the plot beneath the images. For instance, at $14 \mathrm{~nm}$ depth (vertical dashed line), approximately $65 \%$ of the image has a depth below $14 \mathrm{~nm}$ (indicated by the blue shaded area). This procedure cannot account quantitatively for multilayer formation such as the feature circled in red. 


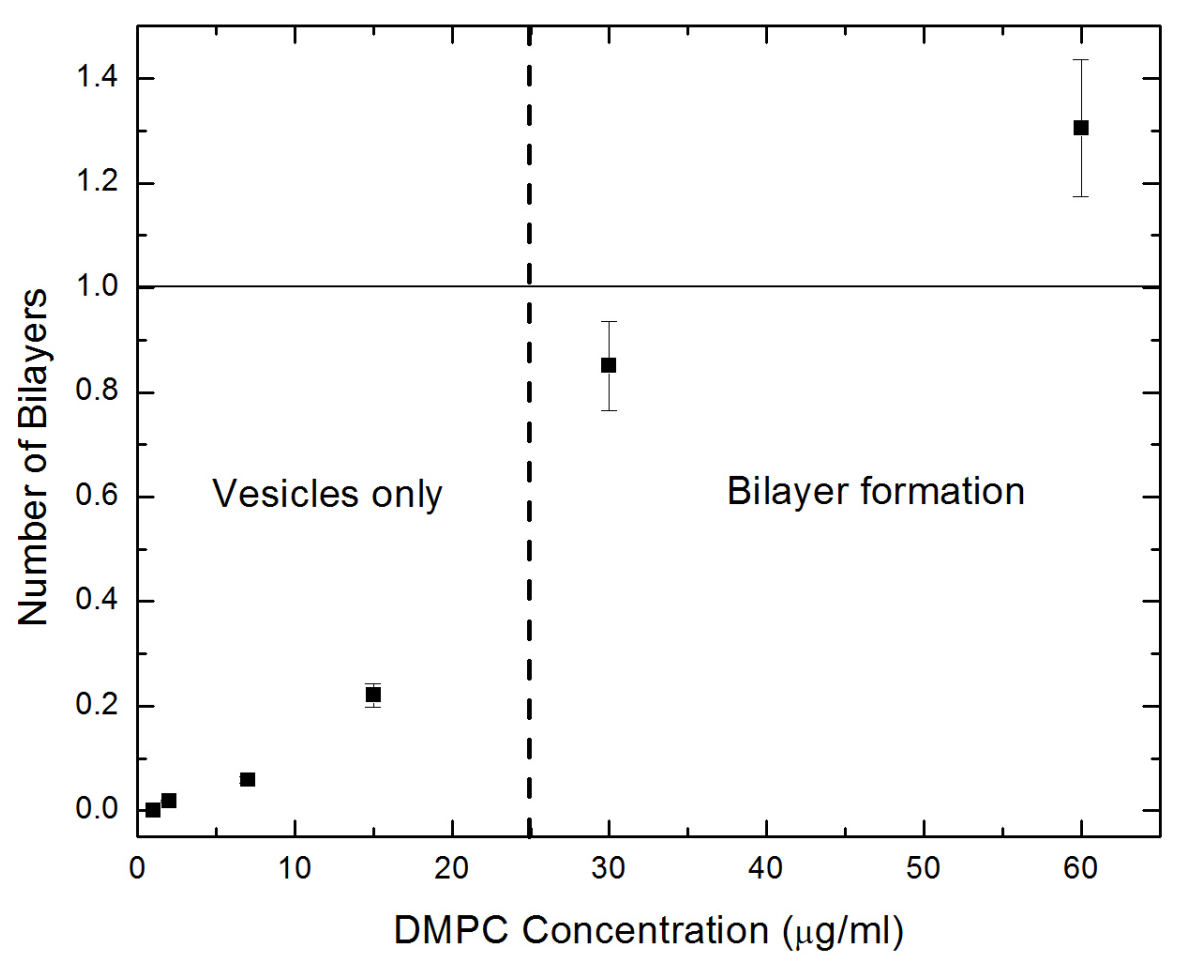

Figure 2.3: DMPC layer coverage vs. lipid solution concentration. Below $30 \mu \mathrm{g} / \mathrm{ml}$ vesicles do not rupture; instead, they adsorb to the surface as spherical vesicles. Above this concentration, lipids form planar bilayers. The linear behavior of coverage vs. concentration at low concentration deviates at concentrations above which vesicles rupture to form bilayers. Multilayer formation is possible at high coverage. For neutron scattering samples, these additional bilayers are removed by rinsing with water. 
centrations. In this regime, vesicles do not interact and some fraction will adsorb to the surface during the incubation time. The adsorbed number is limited by the concentration. However, above a critical concentration, adsorbed vesicles rupture and form planar bilayers. The surface area to mass ratio for planar bilayers is larger than for adsorbed vesicles. As such, the coverage increases faster above the critical concentration.

Figure 2.3 indicates that the minimum lipid concentration to achieve full bilayer coverage is about $30 \mu \mathrm{g} / \mathrm{ml}$. For neutron samples, which require a single full layer, it is necessary to produce samples using concentrations greater than $30 \mu \mathrm{g} / \mathrm{ml}$ (additional layers can be removed by rinsing with water). However, there are other applications for which submonolayer coverage is desired such as measuring the temperature dependence of the membrane thickness by AFM to identify the gel-to-fluid phase transition.

\subsection{Producing Supported DMPG Bilayers}

A vesicle fusion method for depositing the anionic lipid phosphoglycerol onto a silicon oxide substrate has not been reported. Previous efforts have demonstrated the feasibility of the Langmuir-Blodgett and Langmuir-Schaefer method for producing bilayers of anionic lipids; furthermore, a fusion method has been used to deposit layers

on mica $[73,74]$. However, compared to PC lipids, supported anionic lipid films have received little attention.

There are a few reasons why the PC lipid bilayers have received more attention than those of anionic lipids. First, supported PC layers are easier to produce. Second, the gel-to-fluid phase transition of PC vesicles depends only on tempera- 
ture whereas the PG phase diagram depends on lipid concentration, salts, and $\mathrm{pH}$. Third, from a biological perspective, PC lipids are more common than other types of lipids. In mammals, PC lipids compose about 40-60 mol\% of the lipid content of the plasma membrane, with phosphoethanolamine (PE, also zwitterionic) being the second largest component [75]. PG lipids are found in trace amounts in mammalian cells, and while they are more common in bacteria (from whence they derive their evolutionary origin) and plant membranes, the total concentrations of PG lipids do not generally exceed $10 \mathrm{~mol} \%$.

However, from both a physical and a biological perspective there are compelling reasons to study phosphoglycerol lipid membranes. The presence of PG (or other anionic) lipids in the plasma membrane appears to be important for the insertion of membrane proteins [76-78]. Because the gel-to-fluid phase transition of PG lipids depends strongly on salts, their presence in a membrane may also serve as a fluidity regulator. The phase of anionic lipids depends on the local ionic concentration; as such, the concentration of anionic lipids in vesicles may be an important metric for predicting liposome fluidity. Vesicles composed of PG lipids are highly stable and resist aggregation due to the formation of an electric double layer and their very strong, negative $\zeta$ potential [40].

Physically, the supported DMPG system has significantly stronger electrostatic interactions with the surrounding medium than the neutral DMPC lipid. The interpretation of quasielastic neutron scattering data for supercooled bulk water, without additional electrostatic interactions, is still controversial [53]. Moreover, the effect of strong electrostatic interactions on the dynamics of water is a relatively unstudied problem from a neutron scattering perspective [79]. The anionic lipid bilayer provides 
a convenient model system for studying the effect of electrostatic interactions on water dynamics because of the relative simplicity of producing an electric field using the planar supported bilayer lipid geometry compared to an in situ method, such as applying a voltage across two parallel plates.

In an effort to produce supported bilayers of $100 \mathrm{~mol} \%$ DMPG, the following features are noted: first, the strongly negative surface potential of the PG membrane must be overcome by the presence of divalent cations in the buffer. Second, it is preferable to neutralize the DMPG with hydrogen ions instead of heavier counterions because there is indirect evidence that membrane fluidity is increased in this configuration which is a criteria for layer formation [29]. Third, there is overwhelming evidence that deposited DMPG membranes are unstable to vigorous rinsing in water; thus, it is difficult to rinse excess material from the membrane [80-82]. The goal, then, is to produce a sample with the optimum lipid concentration to achieve a single bilayer.

\subsubsection{Sample Preparation of Supported DMPG Bilayers}

$\mathrm{Si}(100)$ wafers coated with their native oxide provide the substrate for all samples. They were prepared as described earlier in this chapter.

The sodium salt of DMPG $\left(\mathrm{C}_{34} \mathrm{H}_{66} \mathrm{O}_{10} \mathrm{PNa}\right)$ from Avanti was prepared by suspending the powder in a $5 \mathrm{ml}$ glass vial in a solution of 65:35:8 chloroform:methanol:water in which the lipids are soluble. Up to $25 \mathrm{mg}$ of powder can be suspended in one vial. The choloroform solution is dried under a slow flow of nitrogen in a fume hood overnight or until the solution is evaporated. A thin DMPG film forms on the inside of the glass vial, which is rehydrated in a buffer solution. The buffer solution 
contains a variable amount of monovalent and divalent salt. The monovalent salt $(\mathrm{KCl})$ is necessary to form the electric double layer around the rehydrated vesicles, which prevents aggregation. The divalent salt $\left(\mathrm{MgCl}_{2}\right)$ acts as an intermediate layer between the silicon oxide substrate and the DMPG bilayer.

The rehydrated lipid solution is heated to $70{ }^{\circ} \mathrm{C}$ and sonicated for $1 \mathrm{hr}$ or until any remaining visible aggregations are removed. The final solution should be clear or milky blue, depending on the concentration. The solution is passed 23-27 times through a $100 \mathrm{~nm}$ filter in a Liposofast filtering apparatus from Avanti. The filtering process involves passing the solution through the filter between two syringes. It is important that an odd number of passes is performed to assure that the final solution arrives in the syringe that did not contain the initial solution.

The filtered solution contains unilamellar vesicles on the order of 100 diameter $\mathrm{nm}$ as determined by dynamic light scattering. After filtering, the solution should be transparent and light blue in color. The solution is diluted in buffer to the desired lipid concentration. The oxidized silicon substrates are submerged in the lipid solution for $1 \mathrm{~h}$. During incubation, vesicles adsorb to the surface and subsequently rupture to form planar layers. Upon removal, the surface is hydrophilic compared to similarly prepared DMPC samples in that a layer of water will coat the surface. Excess solution is evaporated in air for 5-10 min prior to imaging by AFM. No vigorous rinsing is applied as this was found to destroy the layers.

\subsubsection{Parameter Space for DMPG Bilayer Production}

Based on the limited reports in the literature of supported PG bilayers, two main variables in the quantity of adsorbed vesicles were identified. First, the presence of 
divalent cations has been shown to be a necessary condition for vesicle adsorption [35]. The range of divalent cation concentration cited in the literature for successful adsorption of mixed bilayers with high molar \% PG lipids is on the order of 10-20 $\mathrm{mM}[83]$.

Second, high membrane fluidity is a necessary condition for layer formation. Somewhat counterintuitively, a high concentration of divalent cations can result in lower membrane fluidity via the formation of ion bridges between adjacent lipid molecules [37]. Because we expect the formation of layers to be possible only in conditions of high divalent salt concentration, the lipid mobility will also be lower than expected. In order to increase membrane fluidity, we began by investigating vesicle adsorption at low lipid concentrations, which offers the possibility of neutralizing the lipids by free $\mathrm{H}^{+}$in neutral water. Their presence appears to increase membrane fluidity compared to bilayers neutralized by heavier salts $[84,85]$. Evidence of increased fluidity is provided by a decrease in the gel-to-fluid phase transition temperature for high-concentration DMPG dispersions with lower $\mathrm{pH}$ in which preferential $\mathrm{H}^{+}$ion neutralization of the headgroup is believed to occur [29]. At $70{ }^{\circ} \mathrm{C}$, the concentration of $\mathrm{H}^{+}$ions in water is on the order of $10 \mu \mathrm{M}$ due to self-ionization. A DMPG concentration of $15 \mu \mathrm{g} / \mathrm{ml}$ corresponds to $21 \mu \mathrm{M}$; thus, the molar concentration of $\mathrm{H}^{+}$ ions and DMPG molecules are comparable at $70{ }^{\circ} \mathrm{C}$.

Monovalent salt concentrations are known to inhibit aggregation properties of lipid vesicles by binding to the headgroups and increasing the energy barrier for aggregation. In the case of the anionic DMPG vesicles, the vesicles are naturally repulsive due to the strong electrostatic interaction; therefore, the monovalent salt concentration need not be as high as for DMPC in order to prevent aggregation. A 
$25 \mathrm{mM} \mathrm{KCl}$ concentration was found to be optimal for bilayer production (compared to $100 \mathrm{mM} \mathrm{KCl}$ for DMPC lipids), though the effect of this variable was smaller than lipid and divalent salt concentrations. All images in this section are collected after incubating in solution with $25 \mathrm{mM} \mathrm{KCl}$ unless otherwise noted.

\subsubsection{Effect of Divalent Salt Concentration on DMPG Bi- layer Formation}

For the DMPC membrane, a critical lipid concentration of about $30 \mu \mathrm{g} / \mathrm{ml}$ was required in order to achieve full layers. Below $15 \mu \mathrm{g} / \mathrm{ml}$, vesicles adsorbed but did not rupture on the surface. Once full bilayers are achieved, additional lipids adsorb onto the bilayer and result in multilayers or aggregations. The DMPC system has the convenient property that these additional layers can be rinsed off with buffer or water, leaving behind single bilayers. In contrast, DMPG is held weakly on the substrate by a thin layer of divalent ions which screen the highly polar silicon oxide surface. We speculate that rinsing with water disrupts the divalent salt network between the substrate and the DMPG membrane.

The importance of divalent salts is supported by the clear morphological change in Figure 2.4 which shows spherical vesicles rupturing into a planar configuration with higher divalent salt concentration. The images in Figure 2.4 are collected after incubating with a $10 \mu \mathrm{g} / \mathrm{ml}$ lipid concentration, which, for DMPC, corresponded to sub-bilayer coverage. At low $\mathrm{MgCl}_{2}$ concentration (5 mM) in (a), vesicles adsorb on the surface in an approximately hemispherical shape with most having a lateral dimension of 100-200 nm (commensurate with the size of the filter used to prepare the solution). But they do not form planar structures, although several larger aggre- 

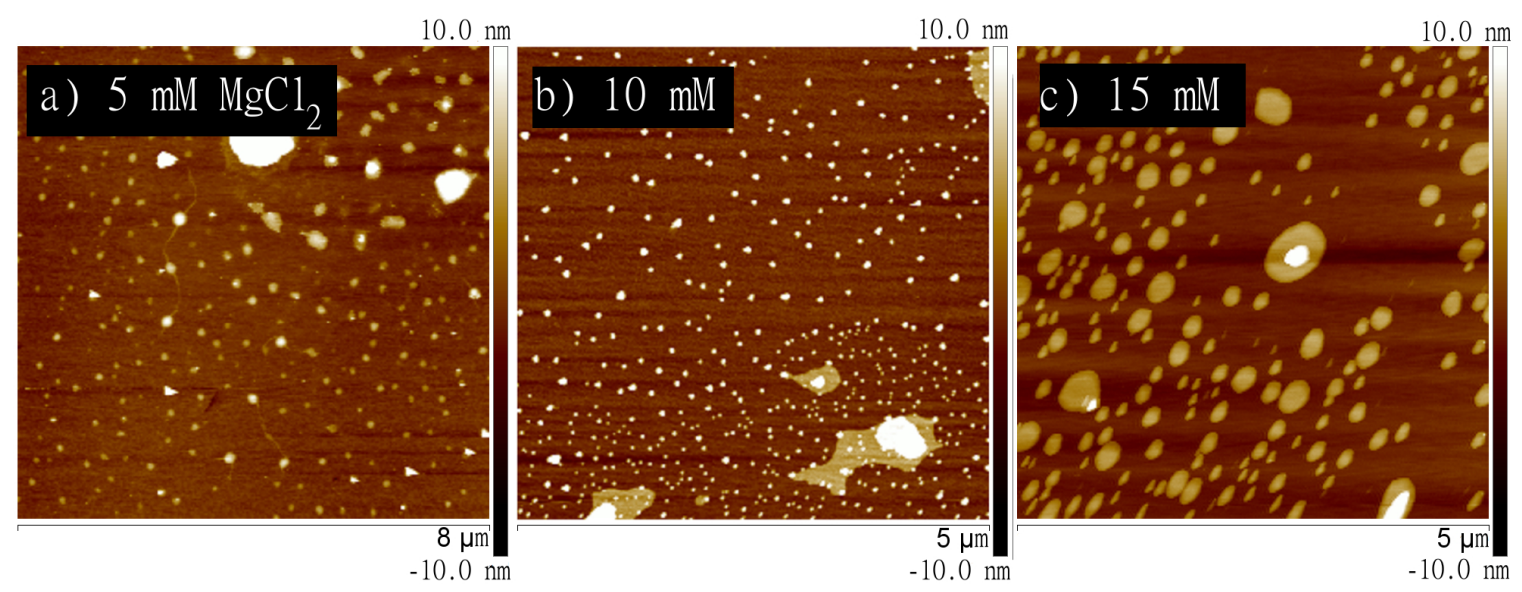

Figure 2.4: AFM images taken in tapping mode of DMPG films deposited from a solution with low lipid concentration. Samples are incubated in $10 \mu \mathrm{g} / \mathrm{ml}$ DMPG solution with (a) $5 \mathrm{mM} \mathrm{MgCl}_{2}$; (b) $10 \mathrm{mM} \mathrm{MgCl}_{2}$; and (c) $15 \mathrm{mM} \mathrm{MgCl}$. At low salt concentrations, vesicles adsorb on the surface but do not rupture. At $10 \mathrm{mM} \mathrm{MgCl}$, planar structures begin to form though most of the material is still in spherical form. In (c), at $15 \mathrm{mM} \mathrm{MgCl}_{2}$, the dominant structure is the planar bilayer.

gations are also noted.

In Figure 2.4(b), with $10 \mathrm{mM} \mathrm{MgCl}_{2}$, most of the adsorbed material is still in the form of hemispherical vesicles. However, some vesicles begin to rupture into planar structures. Finally, at $15 \mathrm{mM} \mathrm{MgCl}_{2}$ in (c), the surface is primarily characterized by these planar structures. The roughly circular shape of these patches indicates that the lipid molecules have high mobility. The bilayer patches in Figure 2.4 are $6.2 \mathrm{~nm}$ thick, similar in height to those of DMPC.

At higher DMPG concentration, the effect of increasing $\mathrm{MgCl}_{2}$ is clear again. As shown in Figure 2.5 and 2.6, spherical vesicles adsorb on the surface but do not rupture to form planar structures. At higher concentrations, these features flatten into planar structures. The presence of a divalent salt layer between the membrane and the substrate reduces the electrostatic repulsion between the negatively charged 

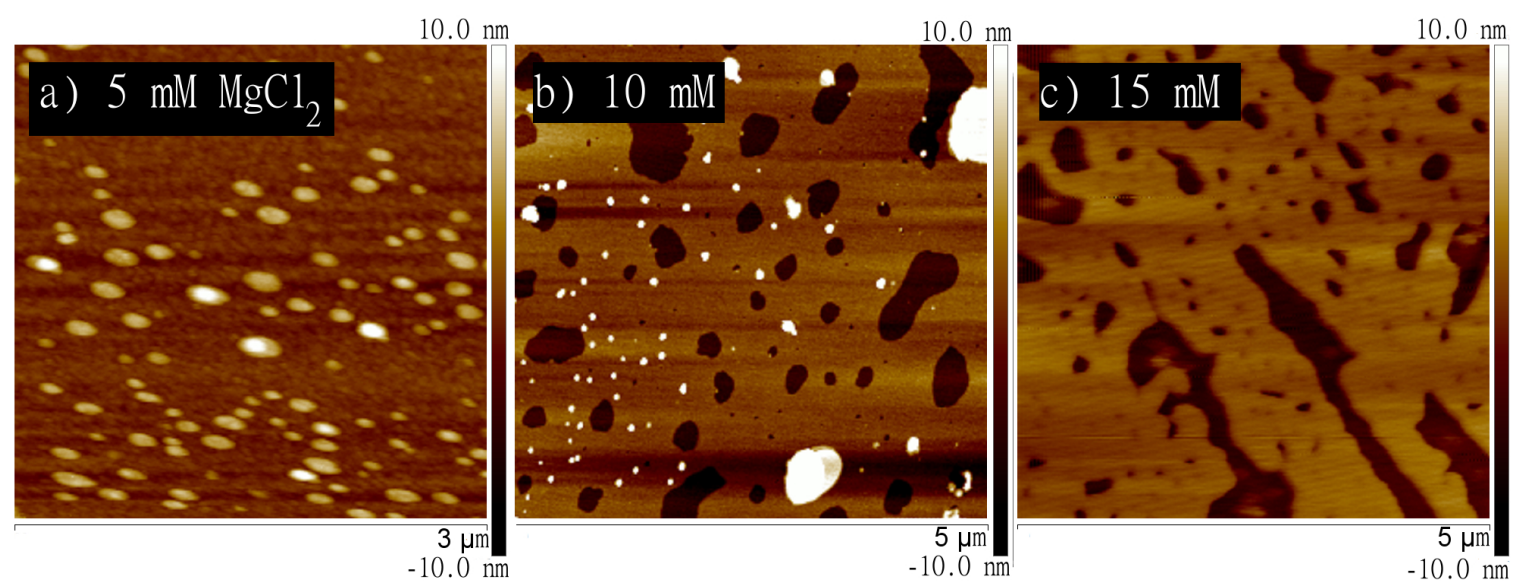

Figure 2.5: AFM images taken in tapping mode of DMPG films deposited from a solution with moderate lipid concentration. Samples are incubated in $15 \mu \mathrm{g} / \mathrm{ml}$ DMPG solution with (a) $5 \mathrm{mM} \mathrm{MgCl}_{2}$; (b) $10 \mathrm{mM} \mathrm{MgCl}$; and (c) $15 \mathrm{mM} \mathrm{MgCl}$. Similar to the case at low lipid concentration, vesicles adsorb but do not rupture at low divalent salt concentrations. In this case, however, planar structures are formed at lower salt concentration [panel (b)], but some vesicles remain in spherical form. Full planar structure formation is achieved with $15 \mathrm{mM} \mathrm{MgCl}_{2}$ at $15 \mu \mathrm{g} / \mathrm{ml} \mathrm{DMPG}$ concentration.

PG bilayer and the silicon oxide surface. In order to achieve mostly bilayer structures, a salt concentration of $15 \mathrm{mM}$ is required. It was found that concentrations above 15 $\mathrm{mM} \mathrm{MgCl}_{2}$ resulted in too much additional material adsorbing to the surface, yielding multi bilayer coverages even at very low lipid concentration.

\subsubsection{Effect of Lipid Concentration on DMPG Bilayer For- mation}

Because the DMPG film is not robust to rinsing with water, our goal was to produce bilayers with as close to complete coverage of a wafer as possible. In the previous section, $15 \mathrm{mM} \mathrm{MgCl}_{2}$ concentration was found to be optimal for the formation of planar structures. Figure 2.7 shows bilayer coverage as a function of lipid concentra- 


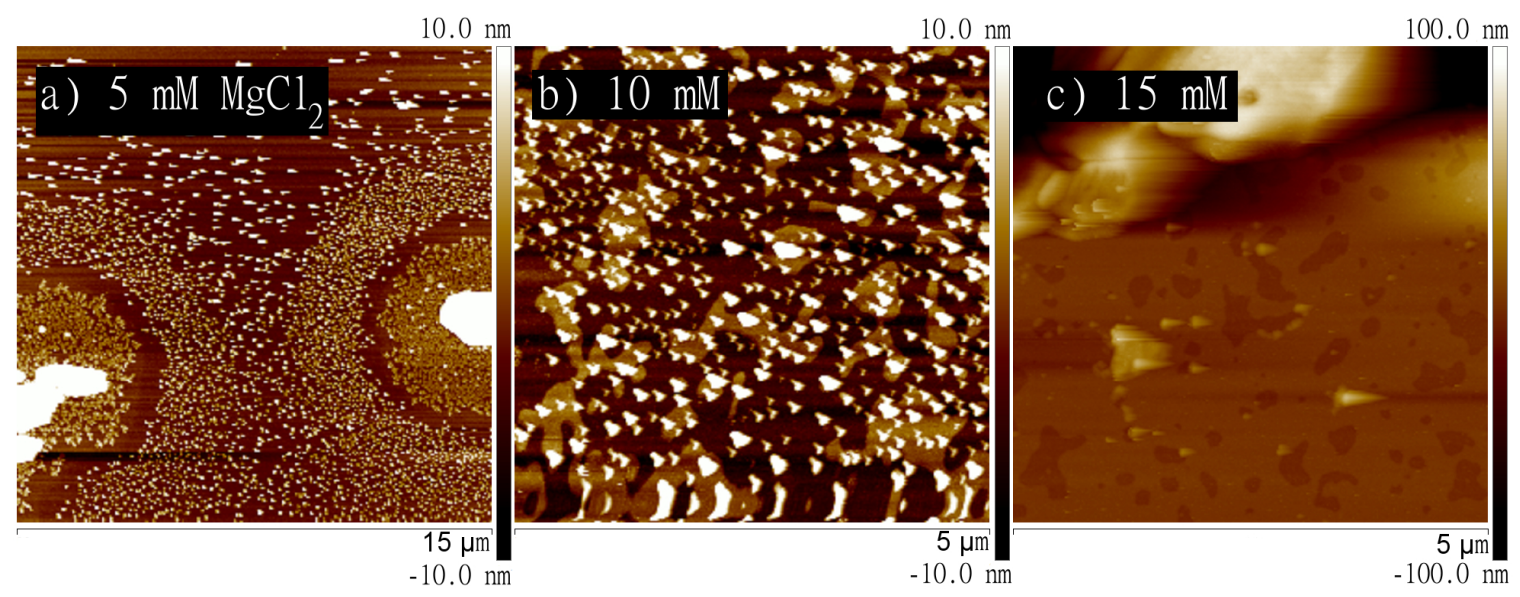

Figure 2.6: AFM images taken in tapping mode of DMPG films deposited from a solution with high lipid concentration. Samples are incubated in $25 \mu \mathrm{g} / \mathrm{ml} \mathrm{DMPG}$ solution with (a) $5 \mathrm{mM} \mathrm{MgCl}_{2}$; (b) $10 \mathrm{mM} \mathrm{MgCl}_{2}$; and (c) $15 \mathrm{mM} \mathrm{MgCl} 2$. In this series of images, a large number of vesicles adsorb to the surface and form planar bilayers at higher salt concentration as in Figure 2.4 and 2.5. However, in panel (c) at $15 \mathrm{mM} \mathrm{MgCl}$, aggregations of excess material begin to form. Note the expanded $z$-scale in panel (c), $\pm 100 \mathrm{~nm}$, emphasizing the amount of additional material.

tion for samples incubated with $15 \mathrm{mM} \mathrm{MgCl}_{2}$ and $15 \mathrm{mM} \mathrm{KCl}$. It demonstrates a similar behavior to that found for DMPC layers: at low coverages, material adsorbs to the surface but does not rupture into planar structures [panel (b)]. Above a critical value (about $7 \mu \mathrm{g} / \mathrm{ml}$ for DMPG compared to $15 \mu \mathrm{g} / \mathrm{ml}$ for DMPC), vesicles rupture into planar structures [panel (c)]. For DMPG, some vesicles do not rupture at this concentration as was seen in Figure 2.5 with the same salt concentrations. Finally, at $15 \mu \mathrm{g} / \mathrm{ml}$, complete bilayer membranes are observed.

Figure 2.8 shows the dependence of bilayer coverage on concentration for DMPG films. At low concentrations, vesicles adsorb to the surface but do not rupture. Above a critical concentration (about $7 \mu \mathrm{g} / \mathrm{ml}$ ), vesicles form planar bilayers. Above $20 \mu \mathrm{g} / \mathrm{ml}$, multiple layers and aggregation of DMPG vesicles occurs.

Full layers are achieved at $15 \mu \mathrm{g} / \mathrm{ml}$ for DMPG, compared to about $30 \mu \mathrm{g} / \mathrm{ml}$ for 


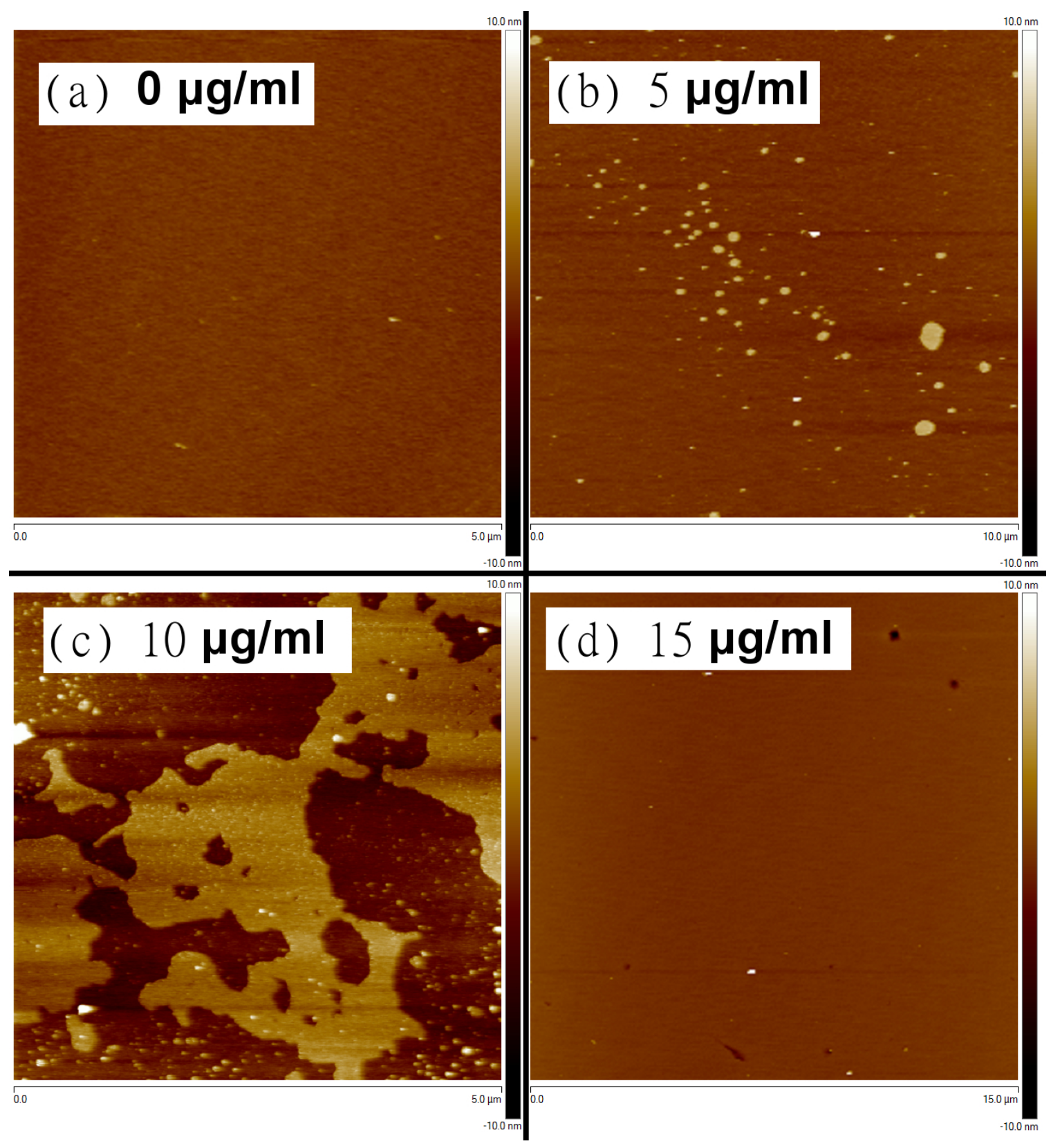

Figure 2.7: AFM images taken in tapping mode showing DMPG bilayer coverage vs. concentration for samples incubated with $15 \mathrm{mM} \mathrm{MgCl} 2$. Samples are incubated with variable lipid concentration: (a) $0 \mu \mathrm{g} / \mathrm{ml}$, (b) $5 \mu \mathrm{g} / \mathrm{ml}$, (c) $10 \mu \mathrm{g} / \mathrm{ml}$, (d) 15 $\mu \mathrm{g} / \mathrm{ml}$. At $5 \mu \mathrm{g} / \mathrm{ml}$, spherically shaped vesicles adsorb and flatten to the surface but do not appear to rupture into planar features. At $10 \mu \mathrm{g} / \mathrm{ml}$, most of the material forms planar structures with a few spherical vesicles. $15 \mu \mathrm{g} / \mathrm{ml}$ was found to be optimum for complete bilayers. Higher concentrations resulted in multilayers and aggregations of lipid material. 


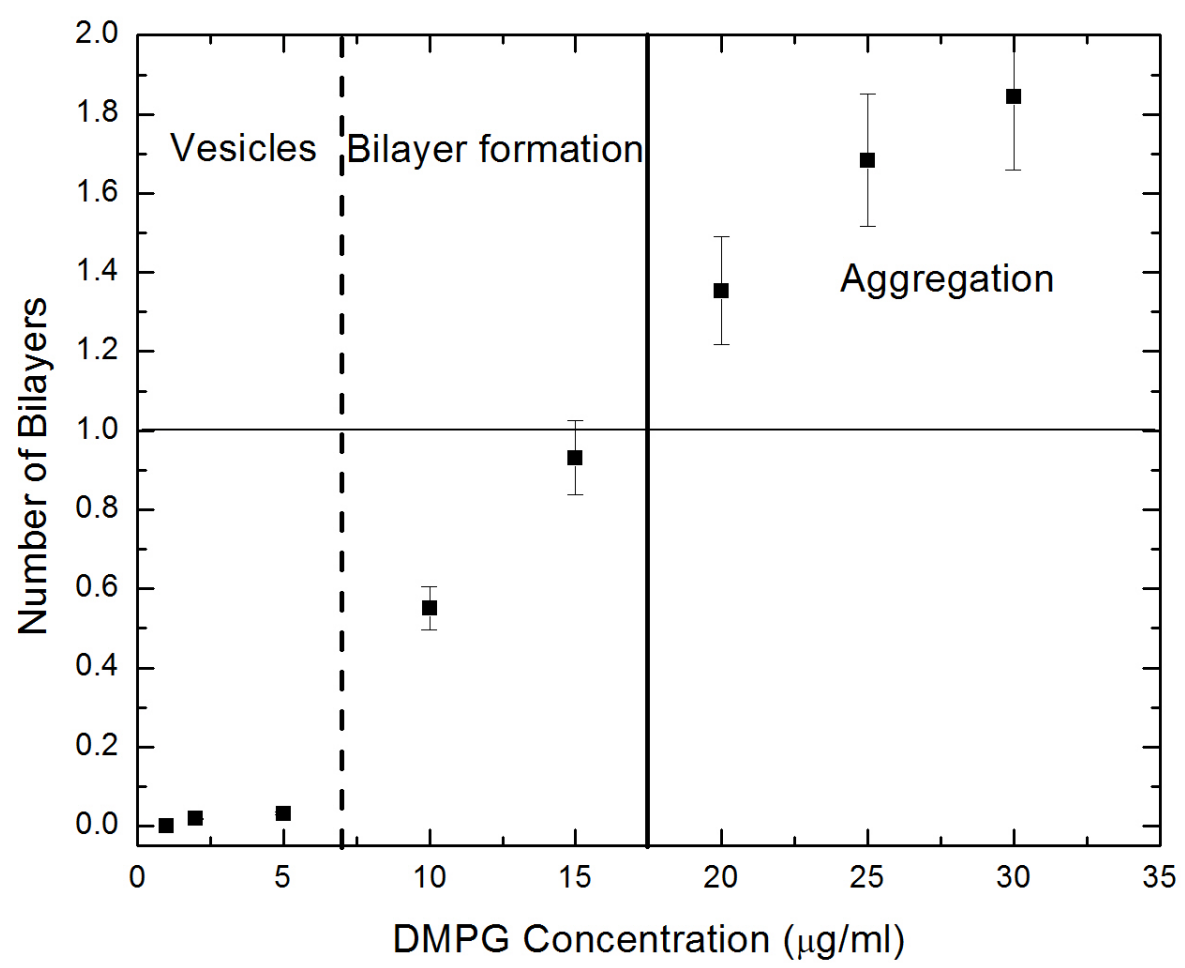

Figure 2.8: DMPG bilayer coverage vs. concentration for samples incubated with 15 $\mathrm{mM} \mathrm{MgCl}_{2}$. The concentration required for full bilayers is smaller for DMPG than for DMPC ( $15 \mu \mathrm{g} / \mathrm{ml}$ compared to $30 \mu \mathrm{g} / \mathrm{ml})$. Furthermore, additional concentration results in multilayers and aggregations which cannot be removed. 
DMPC. Furthermore, the amount of material adsorbed on the surface at a given lipid concentration is higher for DMPG lipids (23\% coverage for DMPC vs. $93 \%$ coverage for DMPG at $15 \mu \mathrm{g} / \mathrm{ml} ; 82 \%$ coverage for DMPC vs $160 \%$ coverage for DMPG at $30 \mu \mathrm{g} / \mathrm{ml})$.

Because the DMPG surface is hydrophilic and the film is not rinsed after incubation, some solution remains on the deposited membrane before evaporating. This

effect may result in additional material adsorbing to the membrane for the same lipid concentration. Another possible explanation for the relatively higher DMPG coverage is the comparatively stronger interaction between the PG headgroup and the $\mathrm{Mg}^{2+}$-primed surface whereas the $\mathrm{PC}$ headgroup interacts with the surface only via van der Waals or dipole electrostatic interactions.

\subsection{The Gel-to-Fluid Phase Transition for Sup- ported Bilayers}

The major advantages of supported lipid layers compared to other sample configurations include the simple planar geometry, the ease of interrogation by a variety of methods, the possibility of relating to molecular dynamics simulation, and the control over lateral pressures and area per lipid when using a Langmuir-Blodgett approach. However, the planar geometry is not a native state and the presence of the substrate may influence the bilayer. In particular, measurements of the lateral diffusion of individual lipid molecules, the formation of domains in mixed lipids, and the thickness of individual layers have all been used to study the effect of the planar support on bilayer function $[23,73,86]$. 
An indirect method of determining the influence of the substrate on the bilayer is investigating structural changes associated with the gel-to-fluid phase transition. In the low temperature gel phase, the alkane tails are in their extended conformation. In the high temperature fluid phase, the tails collapse to a more globular shape through the formation of gauche defects. Thus, the phase of the bilayer can be determined by measuring its thickness as a function of temperature using AFM. This method has also allowed investigation of domain formation in cases where both gel and fluid phases coexist in monospecies bilayers [30].

There are three major reasons for investigating the gel-to-fluid phase transition with AFM prior to our neutron scattering experiments. First, molecular dynamics simulations are performed on free standing bilayers with no substrate. Thus it is important to quantify the influence of the substrate in order to make reasonable comparison between the quasielastic neutron scattering results and simulations.

Second, the quasielastic scattering experiments are performed below physiological temperatures where we assume the bilayer is in the gel phase. We confirm this interpretation with AFM here. In interpreting the neutron result, a possible lateral diffusive motion of the lipids that is possible in the fluid phase can be excluded by knowing the bilayer is in the gel phase.

Third, it is observed that DMPC bilayer homogeneity is sensitive to annealing above the gel-to-fluid phase transition. During the neutron scattering experiments, we examined the effect of annealing the bilayer above the gel-to-fluid phase transition in successive thermal cycles. For DMPC samples in air it is observed (Figure 2.9) that the gel-to-fluid phase transition temperature is shifted upwards to about $50{ }^{\circ} \mathrm{C}$. Therefore, it will be necessary to raise the sample temperature above $50{ }^{\circ} \mathrm{C}$ during 
the neutron scattering experiments to investigate the effect of annealing.

In Figure 2.9, we plot the bilayer thickness as a function of temperature under nitrogen gas and water vapor for DMPC and DMPG bilayers. Water is evaporated from a reservoir to provide an approximation of the humidity conditions in our "Wet" neutron scattering samples (black points). Nitrogen gas is flowed over the samples at a slow rate $(0.5 \mathrm{ml} / \mathrm{s}$, red points) to simulate our "Dry" samples. We see that the dry conditions result in an upward shift of the temperature at which the gel-to-fluid phase transition occurs. By establishing an upper bound on the gel-to-fluid phase transition temperature, we can select an appropriate temperature for annealing our neutron scattering samples.

The bilayer thickness monotonically decreases with increasing temperature for both membranes under both conditions. Under nitrogen, the gel-to-fluid phase transition is observed at higher temperature for both lipids than under water vapor. The bilayer thickness does not reach a limiting value until about $70{ }^{\circ} \mathrm{C}$ for both lipids. Under water vapor, the DMPC lipid is in the fluid phase at $55{ }^{\circ} \mathrm{C}$, while the DMPG lipid remains in the gel phase until $70{ }^{\circ} \mathrm{C}$. We speculate that the DMPG lipid remains in the gel phase to higher temperature due to the stronger substrate-bilayer and headgroup-headgroup interactions both mediated by the presence of divalent cations. 


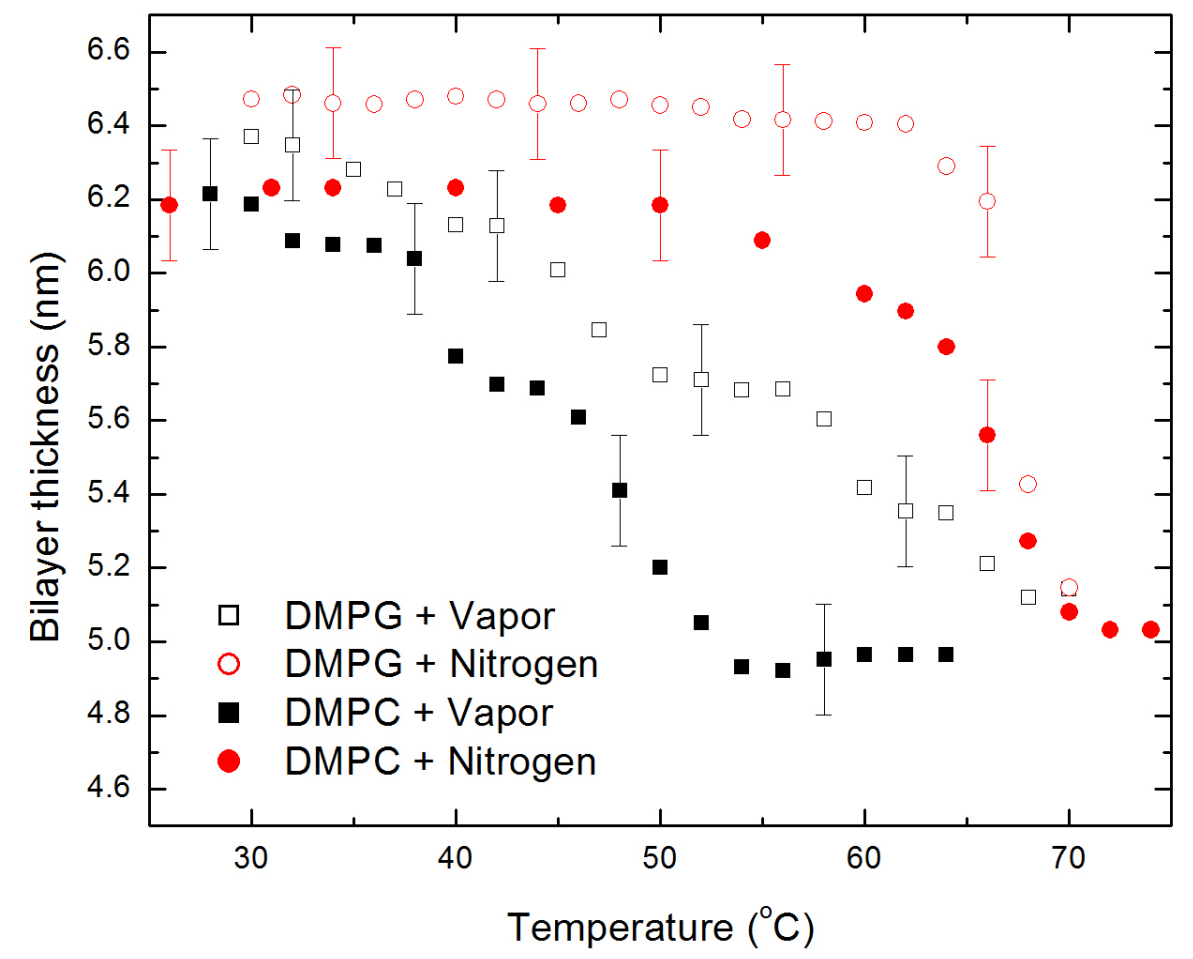

Figure 2.9: AFM measurements in tapping mode showing DMPG (open symbols) and DMPC (closed symbols) membrane thickness vs. temperature under water vapor (black points) and nitrogen gas (red points). The gel-to-fluid phase transition for DMPG bilayers appears to be shifted to higher temperatures than DMPC under both conditions. 


\subsection{Complete Bilayers for Neutron Scattering Ex- periments}

For quasielastic neutron scattering experiments we desire to have complete lipid bilayers. Complete bilayers are featureless on large $(>\mu \mathrm{m})$ scale; as such, it is difficult to identify the presence of the bilayer based on topology alone due to its similarity to the silicon oxide.

One method for confirming the presence of the bilayer is to mechanically expose the substrate by scratching with a razor blade. A typical razor blade may be on the order of $200 \mu \mathrm{m}$ wide at its edge and will contaminate the sample. However, by imagining near the boundary of the scratch it is possible to observe a region such as in Figure 2.10 wherein lipid material has been removed, exposing the silica. A typical line section reveals the bilayer thickness to be on the order of $6 \mathrm{~nm}$, consistent with the thickness measurement on sub-monolayer coverage bilayers.

A less invasive method of confirming the presence of the bilayer is to identify regions of displaced material, as in Figure 2.11. Here, occasional defects in the layer can be found which provide a measurement to the silica substrate. In both DMPC and DMPG samples the bilayer thickness is measured to be on the order of $6 \mathrm{~nm}$, also consistent with the thickness measurement of sub-monolayer bilayers. The DMPC sample measured in Figure 2.10(a) and Figure 2.11(a) was produced simultaneously with the Wet 1 sample measured with quasielastic neutron scattering in Chapter 3. The DMPG sample in Figure 2.10(b) and Figure 2.11(b) was produced with the Wet 1 DMPG sample discussed in Chapter 4. As such, these images are representative of neutron scattering samples. 

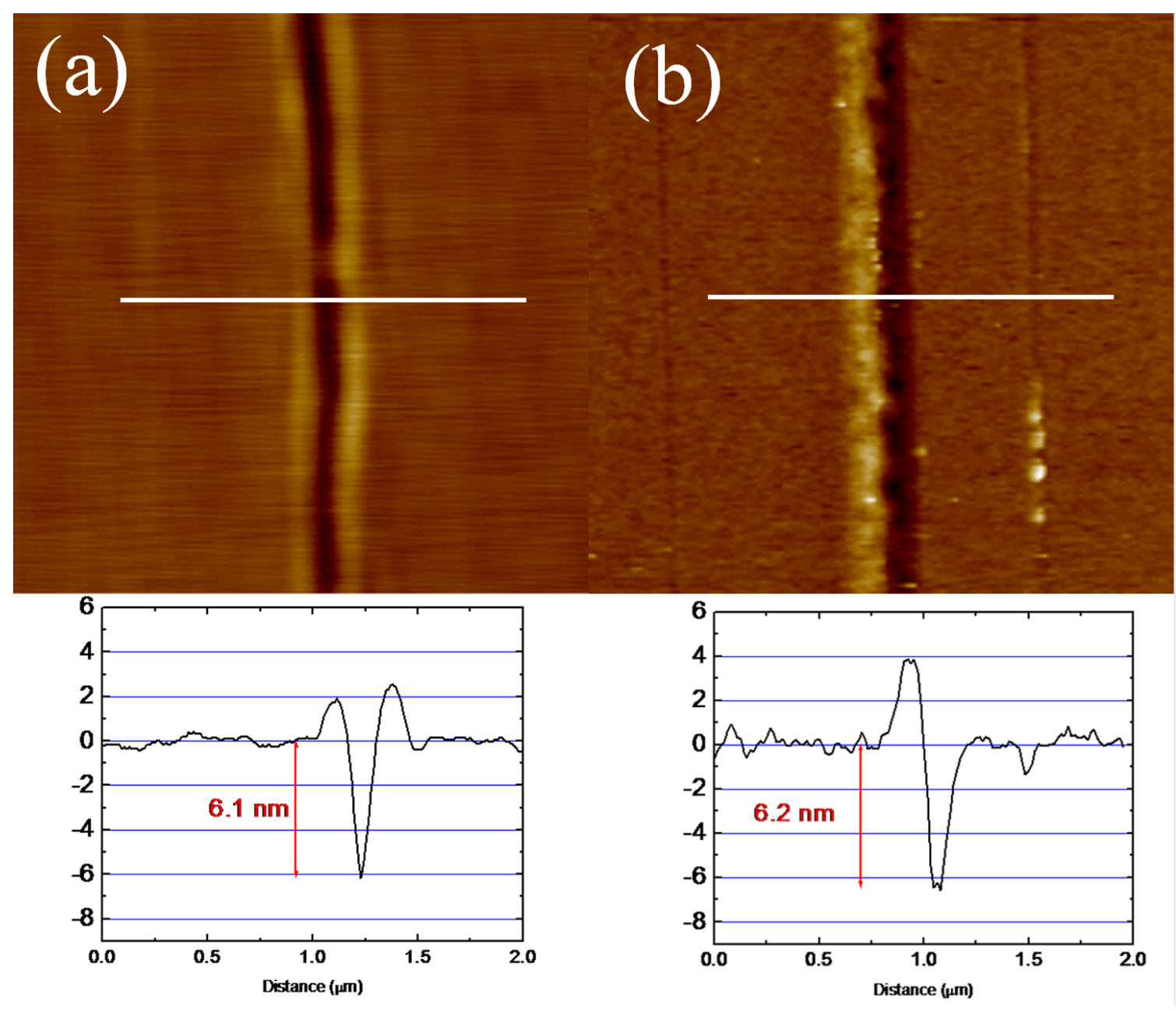

Figure 2.10: AFM measurements in tapping mode in air at room temperature of full DMPC bilayers [panel (a)] and DMPG bilayers [panel (b)]. Full DMPC layers are produced by incubating in a solution of $200 \mathrm{mg} / \mathrm{ml} \mathrm{DMPC,} 5 \mathrm{mM} \mathrm{MgCl} 2$, and 100 $\mathrm{mM} \mathrm{KCl}$ at $55{ }^{\circ} \mathrm{C}$ for $1 \mathrm{~h}$. Samples are then rinsed in distilled water prior to imaging. Full DMPG layers are produced by incubating in a solution of $15 \mu \mathrm{g} / \mathrm{ml}$ DMPC, 15 $\mathrm{mM} \mathrm{MgCl}$, and $15 \mathrm{mM} \mathrm{KCl}$ at $65^{\circ} \mathrm{C}$ for $1 \mathrm{~h}$. No rinsing is applied to the DMPG samples. The silica surface is exposed by scratching the sample with a razor blade. 

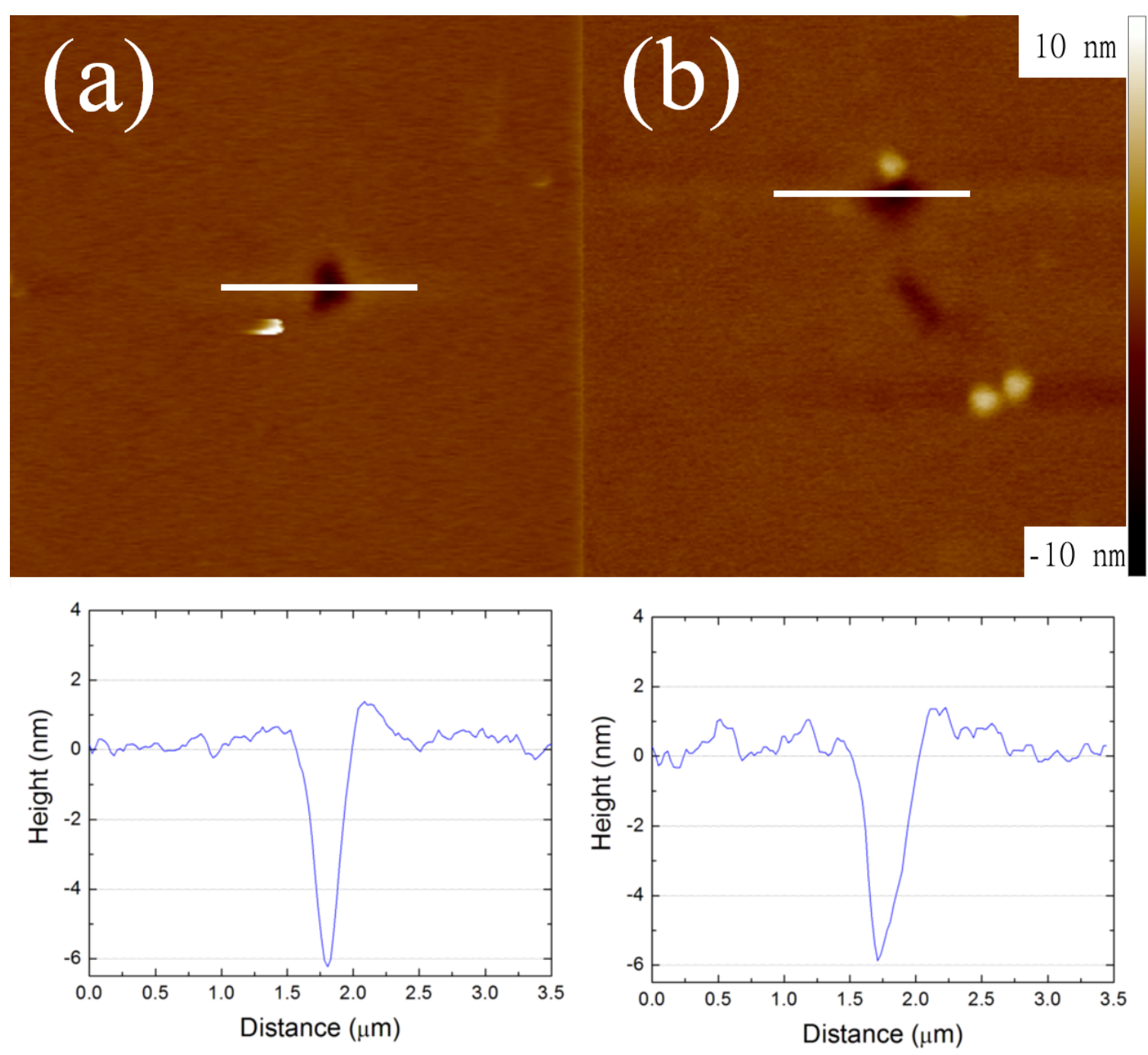

Figure 2.11: AFM measurements in tapping mode in air at room temperature of full DMPC bilayers [panel (a)] and DMPG bilayers [panel (b)]. Full DMPC layers are produced by incubating in a solution of $200 \mathrm{mg} / \mathrm{ml} \mathrm{DMPC}, 5 \mathrm{mM} \mathrm{MgCl} 2$, and 100 $\mathrm{mM} \mathrm{KCl}$ at $55^{\circ} \mathrm{C}$ for $1 \mathrm{~h}$. Samples are then rinsed in distilled water prior to imaging. Full DMPG layers are produced by incubating in a solution of $15 \mu \mathrm{g} / \mathrm{ml}$ DMPC, 15 $\mathrm{mM} \mathrm{MgCl}$, and $15 \mathrm{mM} \mathrm{KCl}$ at $65{ }^{\circ} \mathrm{C}$ for $1 \mathrm{~h}$. No rinsing is applied to the DMPG samples. Occasional defects in the bilayer can be observed. Some lipid material is displaced, revealing the silica substrate. The depth of the displacement is $6.1 \mathrm{~nm}$ in (a) and $5.9 \mathrm{~nm}$ in (b). 


\section{Chapter 3}

\section{Neutron Scattering from DMPC Bilayer Lipid Membranes}

\subsection{Introduction}

In this chapter, we will discuss the results of neutron scattering experiments performed on the High-Flux Backscattering Spectrometer (HFBS) at the National Institute for Standards and Technology Center for Neutron Research (NCNR) in Gaithersburg, MD and the Backscattering Spectrometer (BASIS) at the Spallation Neutron Source at Oak Ridge National Laboratory in Oak Ridge, TN.

Elastic neutron scattering data as a function of temperature are presented first. The elastic scans provide preliminary insight into the water structure and dynamics, provide a characterization of sample homogeneity, and quantify the hydration of 
the samples. Extending these results, full quasielastic spectra will be presented and discussed from which a more detailed picture of the water motion emerges.

The quasielastic measurements reveal three types of water present in these samples of single-supported DMPC bilayers. First, a large amount of supercooled bulk-like water whose dynamics is similar to bulk supercooled water is observed. However, a second, smaller amount of water which is closer to the membrane but not directly hydrogen bonded to it appears to remain mobile to lower temperatures. This "confined" water displays slower dynamics than bulk supercooled water and extends upwards from the membrane by about $10 \mathrm{~nm}$. Finally, an even smaller amount of water, representing 8-10 water molecules per lipid, undergoes a slower, bounded motion in concert with motion of the lipid tails and head groups. The number of bound water molecules per lipid has been quantified here for the first time using neutron scattering, a confirmation of previous NMR and molecular dynamics results $[1,65,87]$.

\subsection{DMPC Neutron Sample Preparation}

Elastic and quasielastic neutron scattering experiments were performed on singlesupported bilayers of zwitterionic DMPC. The DMPC samples were hydrated with variable amounts of water and different deuteration conditions were used. Either the hydrocarbon lipid tails or the hydrating water was replaced with deuterated hydrocarbons or $\mathrm{D}_{2} \mathrm{O}$, respectively. Replacing hydrogen with an isotope, deuterium, 


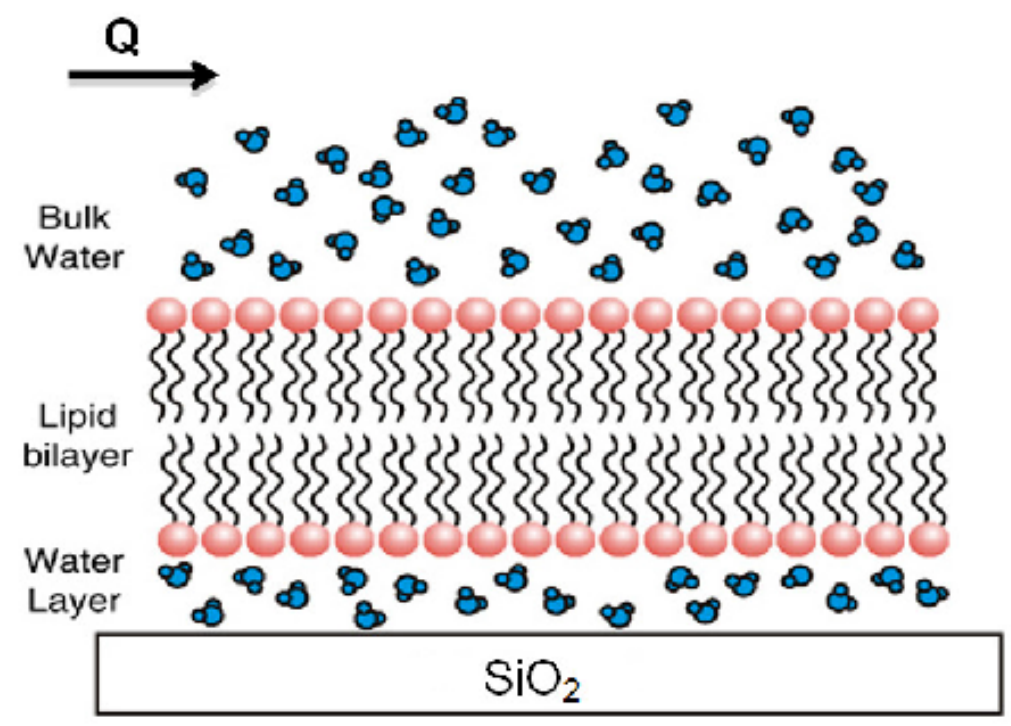

Figure 3.1: Sketch of a hydrated single-supported lipid bilayer as suggested by Ref. [6].

renders that component of the system invisible to neutrons in this experiment because the incoherent cross section of $\mathrm{H}(80.3 \mathrm{~b})$ is much larger than for $\mathrm{D}(2.05 \mathrm{~b})$. In this way, we can selectively probe certain parts of the system independently.

Samples for these experiments were produced using methods developed in Chapter 2 except the oxidized silicon wafers were circular 2 inches in diameter and the lipid concentration in the deposition solution was $0.5 \mathrm{mg} / \mathrm{ml}$. No significant differences in membrane morphology were noted with deuterated lipids; thus, the sample production procedure was identical for protonated and deuterated lipid samples.

After membrane deposition on 100 silicon wafers for each sample, one of three additional steps to modify the hydration of the system were used. For high hydration samples, wafers are annealed for 3 days at $65{ }^{\circ} \mathrm{C}$ to evaporate any bulk water and 
improve layer homogeneity. Then, the dry wafers are sealed in a cylindrical aluminum sample can with a $508 \mu \mathrm{m}$ wall thickness and neutron window $35 \mathrm{~mm}$ high. A $120 \mu \mathrm{l}$ puddle of water is added to the can. Once sealed with an indium O-ring, the puddle evaporates, filling the enclosed volume and rehydrating the sample.

For low hydration samples, wafers are not annealed and mounted directly in the sample can without the addition of a puddle of bulk water. For DMPC, the surface of which is macroscopically hydrophobic after deposition, this procedure represents less water than the high hydration case. Finally, samples of very low hydration ("superdry" samples) are prepared by annealing for 3 days at $65{ }^{\circ} \mathrm{C}$ prior to sealing in the aluminum can with no additional bulk water. Under this condition, only a thin shell of hydration water remains. Samples produced with tail-deuterated $\mathrm{D}_{54}$ lipids were prepared identically.

The primary experimental variables are the sample hydration and deuteration contrast. In the previous section, we outlined three methods for producing samples of variable hydration; however, these methods do not provide a technique for quantitatively determining the amount of water in each sample. Instead, the amount of water in each sample is inferred by measuring the increase in total elastic scattering at low temperature. The details of this calculation rely on a calibration using a sample of known hydrogen content as described in Appendix A.

The amount of water in each sample can be expressed in several ways. The total 
Table 3.1: Deuteration and water content for DMPC samples

\begin{tabular}{|l|l|l|}
\hline Sample Name & Lipid Deuteration & Water Deuteration \\
\hline Wet 1 & $\mathrm{p}-72$ & $\mathrm{H}_{2} \mathrm{O}$ \\
\hline Wet 2 & $\mathrm{p}-72$ & $\mathrm{H}_{2} \mathrm{O}$ \\
\hline Dry & $\mathrm{d}-54+\mathrm{p}-18$ & $\mathrm{H}_{2} \mathrm{O}$ \\
\hline Superdry & $\mathrm{p}-72$ & $\mathrm{H}_{2} \mathrm{O}$ \\
\hline Membrane & $\mathrm{p}-72$ & $\mathrm{D}_{2} \mathrm{O}$ \\
\hline
\end{tabular}

\begin{tabular}{|l|c|c|c|}
\hline Sample Name & Water Volume $(\mu \mathrm{l})$ & Water molecules per Lipid & Film Thickness $(\AA)$ \\
\hline Wet 1 & 58.1 & 1,556 & 1,132 \\
\hline Wet 2 & 42.7 & 1,140 & 829 \\
\hline Dry & 5.73 & 154 & 111 \\
\hline Superdry & - & - & - \\
\hline Membrane & - & - & - \\
\hline
\end{tabular}

amount of water can be given in terms of a volume (assuming bulk density), as a ratio to the number of lipid molecules, or in terms of a film thickness. The film model is thought of as a cylindrical volume containing water at bulk density with cross sectional area equal to that of the wafer surface (a 2 in diameter circle) and given thickness. It is imagined that the cylindrical film extends upwards from the planar membrane surface uniformly on both sides of each of the 100 wafers. However, the actual morphology of the water is not known in these samples. The water may exist in bulk droplets or another morphology. We use the film model for simplicity.

The list presented in Table 3.1 is representative. A full list of samples including samples whose data is omitted is presented in Appendix A. Other samples that are not presented here were also measured. They include two preliminary DMPC samples, one produced with $120 \mu \mathrm{l} \mathrm{D}$ O having been annealed for 3 days prior to sealing and 
a second sample identical but with $\mathrm{H}_{2} \mathrm{O}$. Both samples were measured in August of 2009 on the HFBS, but the results are omitted here. Another sample of 100 clean silicon wafers with no lipids and $120 \mu \mathrm{l} \mathrm{H}_{2} \mathrm{O}$ was measured on the HFBS in November 2012. The results from these samples do not conflict with or extend the discussion in this chapter.

\subsection{Elastic Neutron Scattering from Supported DMPC Membranes}

In this section, we detail the results of elastic incoherent neutron scattering measurements on the samples described above as a function of temperature. The intensity of the elastic scattering in these measurements is proportional to the amount of water static on the time scale of the instrument. These results provide the foundation upon which more detailed quasielastic measurements may be analyzed, which we will discuss in the next section.

Elastic neutron scattering intensities as a function of temperature are collected on the High-Flux Backscattering Spectrometer (HFBS) at the NIST Center for Neutron Research in Gaithersburg, MD. This instrument has an energy resolution of $\pm 1.0 \mu \mathrm{eV}$, corresponding to a time scale of $4 \mathrm{~ns}$. The scattering from hydrated lipid samples is primarily incoherent scattering from hydrogen nuclei. Therefore, the intensity is 
proportional to the number of hydrogen nuclei (mostly originating from water) that move on a time scale slower than $4 \mathrm{~ns}$.

In Figure 3.2, we plot the intensity of neutrons scattered elastically from three DMPC samples, two prepared identically [panel (a), samples Wet 1 and Wet 2] and one with approximately 10 times less water [panel (b), Dry sample], summed over all $Q$ values. At high temperature, the intensity from all samples is primarily incoherent scattering from silicon. A possible component may originate from coherent scattering from silicon; however, the coherent contribution is likely negligible for two reasons. First, the coherent cross section of silicon is 1,000 times smaller than the incoherent cross section. Second, the incident neutron wavelength used in this experiment is too large to observe Bragg peaks in silicon.

Furthermore, a portion of the incoherent scattering from mobile water is collected at high temperature. For low $Q$, this portion is a significant component; however, at higher $Q$ the intensity of the quasielastic water component inside the elastic window decreases as the quasielastic scattering broadens.

The data is normalized such that the intensity at $270 \mathrm{~K}$ on cooling is unity. As the samples are cooled (blue points), a freezing step is observed in the Wet 1 and Wet 2 samples around 262-265 K. This intensity originates from incoherent scattering from hydrogen in water as it freezes into the elastic energy window. Further cooling results in a continuous and monotonic increase in elastic intensity with decreasing temper- 


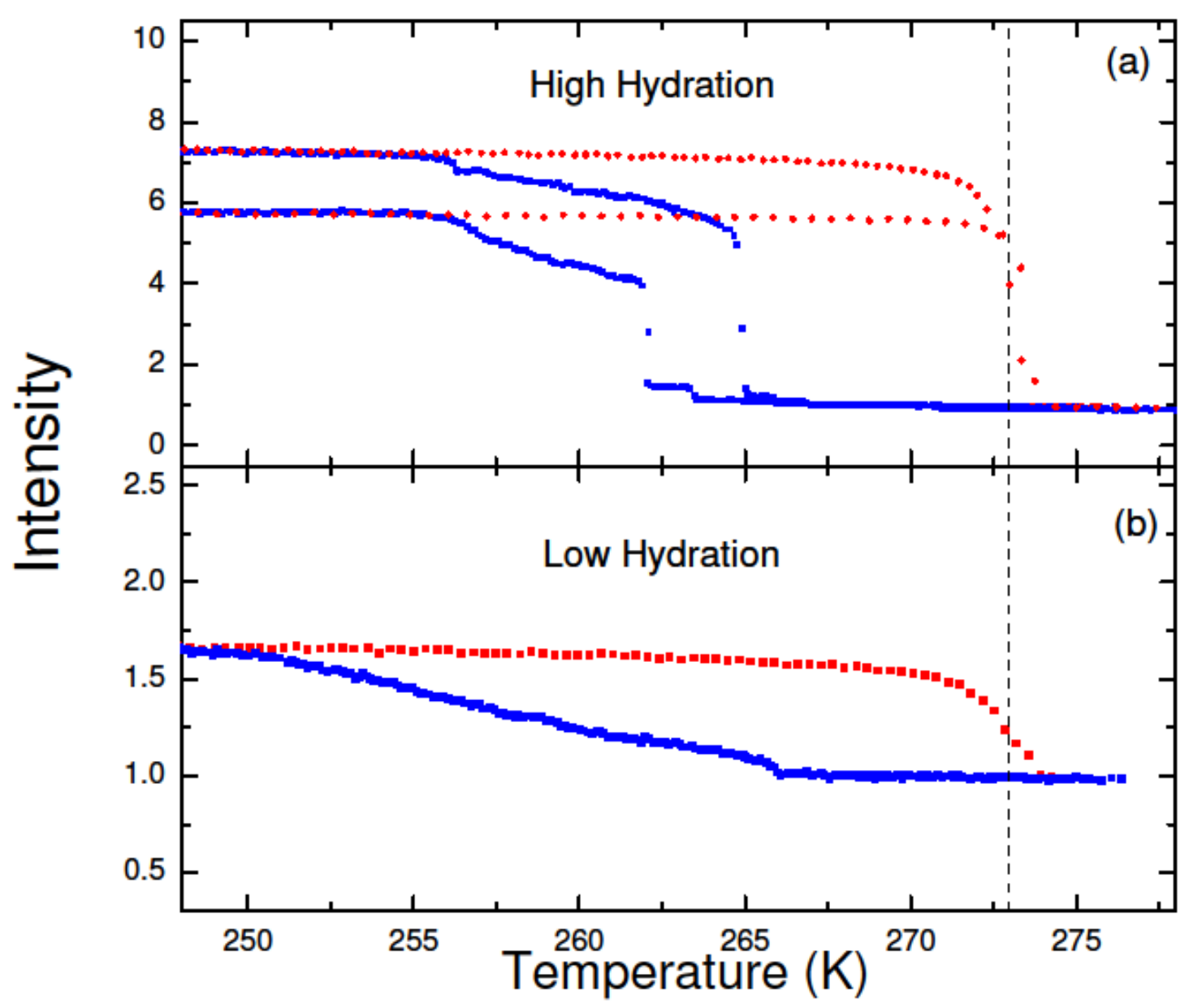

Figure 3.2: Elastic neutron scattering intensity as a function of temperature for DMPC samples: (a) Wet 1, top curve; Wet 2, lower curve and (b) Dry, as collected on the HFBS. Blue points indicate cooling and red points indicate heating. The vertical line represents the bulk melting point of water at $273 \mathrm{~K}$. Wet samples demonstrate a vertical step in the temperature range between 260 and $265 \mathrm{~K}$, indicating the freezing of a large amount of bulk-like water on cooling. Further cooling results in a gradual increase in intensity as additional water subsequently freezes. The low hydration sample does not show the bulk-like step due to having less water. Both samples [(a) and (b)] demonstrate complete melting near the bulk melting point. Data are collected with a temperature ramp of $0.04 \mathrm{~K} / \mathrm{min}$ on cooling and $0.1 \mathrm{~K} / \mathrm{min}$ on heating at 5 minutes per point. Neutron counts in all 16 detectors corresponding to different $Q$ values in the range of $0.25-1.75 \AA^{-1}$ are summed and normalized to the monitor. 
ature until all motion is frozen around $255 \mathrm{~K}$. The Dry sample also demonstrates a continuous monotonic increase in intensity but without the freezing step near $265 \mathrm{~K}$. Upon heating (red points), all samples demonstrate melting, indicated by an abrupt decrease in elastic intensity near the bulk point of $273 \mathrm{~K}$ indicated by the vertical black dashed line.

DMPC layers deposited on silicon wafers experience a morphological change when annealed above the gel-fluid phase transition. The major effect is to fill in holes or defects in the DMPC layer, which produces a more homogeneous surface. Therefore, one might expect the elastic intensity of the same sample of DMPC to change if the sample is annealed above the gel-fluid phase transition. Figure 3.3 shows successive thermal cycles of the high-hydration DMPC sample presented in Figure 3.2 (Wet 2). Cycle 2 shows good reproducibility with the initial thermal cycle after the sample is heated to $280 \mathrm{~K}$.

While cooling during cycle 1, three small freezing steps can be distinguished (at $271 \mathrm{~K}, 267 \mathrm{~K}$, and $263 \mathrm{~K}$ ) prior to a main freezing step at $262 \mathrm{~K}$. In cycle 2, only a single small step is still observed at $270 \mathrm{~K}$ in addition to the main freezing transition at $265 \mathrm{~K}$. The smaller substeps are likely due to an onset of freezing on some of the wafers due to inhomogeneities in the 100-wafer stack or local nucleation sites near regions of exposed silicon. After annealing, a larger fraction of the 100 wafers have defect-free DMPC layers on them, which is evidenced by a single, larger freezing 


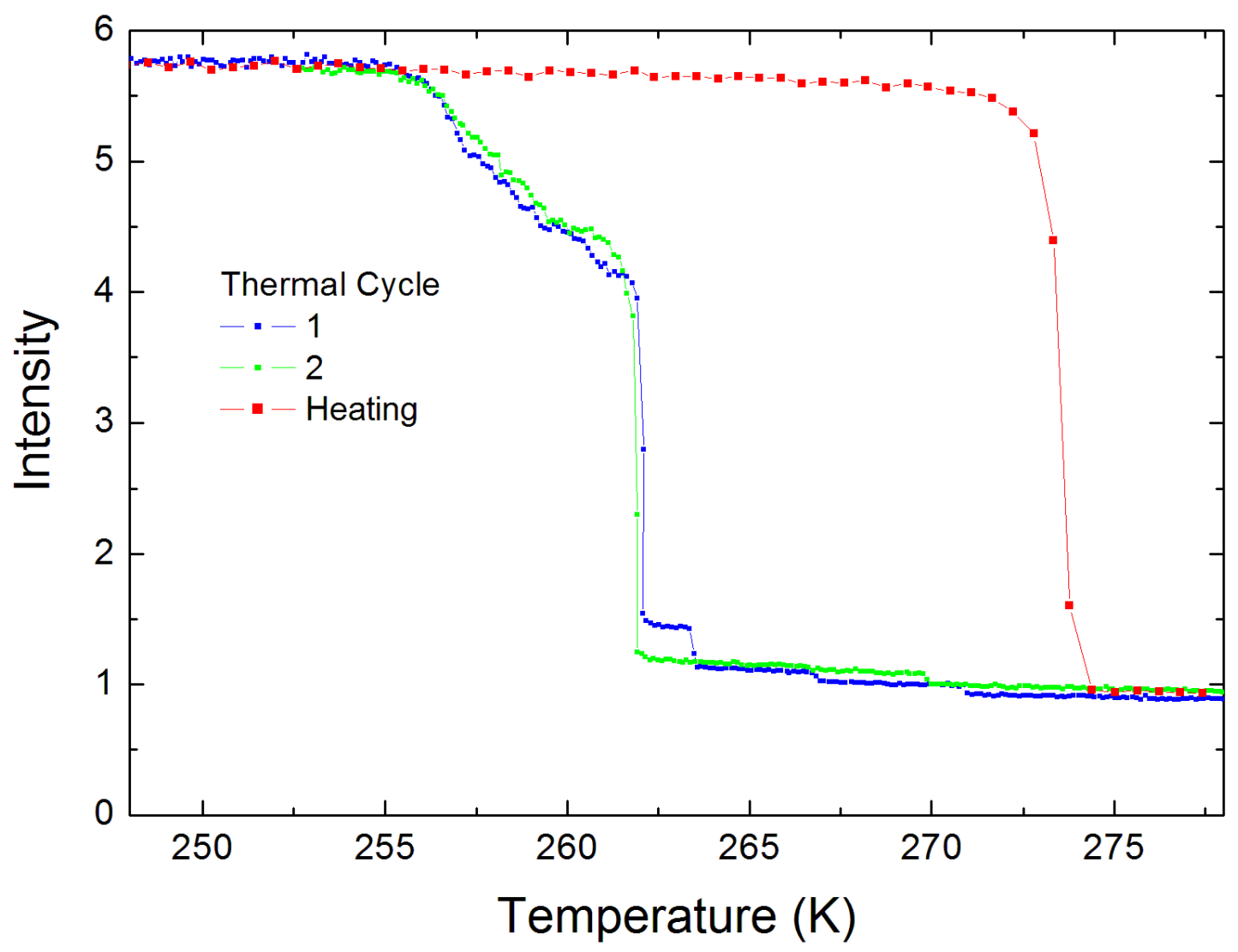

Figure 3.3: Elastic neutron scattering intensity as a function of temperature for the Wet 2 DMPC sample [bottom curve in Figure 3.2(a)] for two cooling thermal cycles collected on the HFBS. The red curve represents heating for cycle 1. Heating for cycle 2 reproduces this curve and is omitted. Cycle 1 on cooling (blue points) demonstrates small substeps at $263 \mathrm{~K}, 267 \mathrm{~K}$, and $271 \mathrm{~K}$ prior to the main freezing transition at 262 K. Cycle 2 (green points) reproduces the main freezing step; however, there is only one smaller substep at $270 \mathrm{~K}$. The substeps are attributed to inhomogeneities between the wafers in the 100-wafer stack. After undergoing a thermal cycle, it is proposed that cracks or holes in the membrane may anneal out, increasing sample homogeneity. Data are collected with a temperature ramp of $0.04 \mathrm{~K} / \mathrm{min}$ on cooling and $0.1 \mathrm{~K} / \mathrm{min}$ on heating at 5 minutes per point. Neutron counts from all 16 detectors corresponding to different $\mathrm{Q}$ values are summed and normalized to the monitor. 
transition at $265 \mathrm{~K}$. The heating curves do not differ significantly for DMPC layers upon successive thermal cycles and thus only a single representative heating curve is included.

An even more dramatic annealing effect is shown in Figure 3.4, which plots successive thermal cycles of the Wet 1 sample shown in Figure 3.2. This measurement is performed on the BASIS spectrometer which has a coarser energy resolution of 3.5 $\mu \mathrm{eV}$, corresponding to a time scale of $1 \mathrm{~ns}$; but the elastic intensity here is nevertheless proportional to the quantity of frozen water. The first thermal cycle (red points) did not demonstrate a sharp freezing transition but instead showed a monotonic increase in intensity during cooling which may be attributed to large inhomogeneities within the wafer stack. After annealing at $335 \mathrm{~K}$, above the gel-fluid phase transition, for $2 \mathrm{~h}$, the cooling curve (green points) evolves into a freezing step and a continuous freezing region below $267 \mathrm{~K}$. This behavior is again attributed to greater homogeneity within the wafer stack. As before, the heating curve does not differ significantly between thermal cycles. 


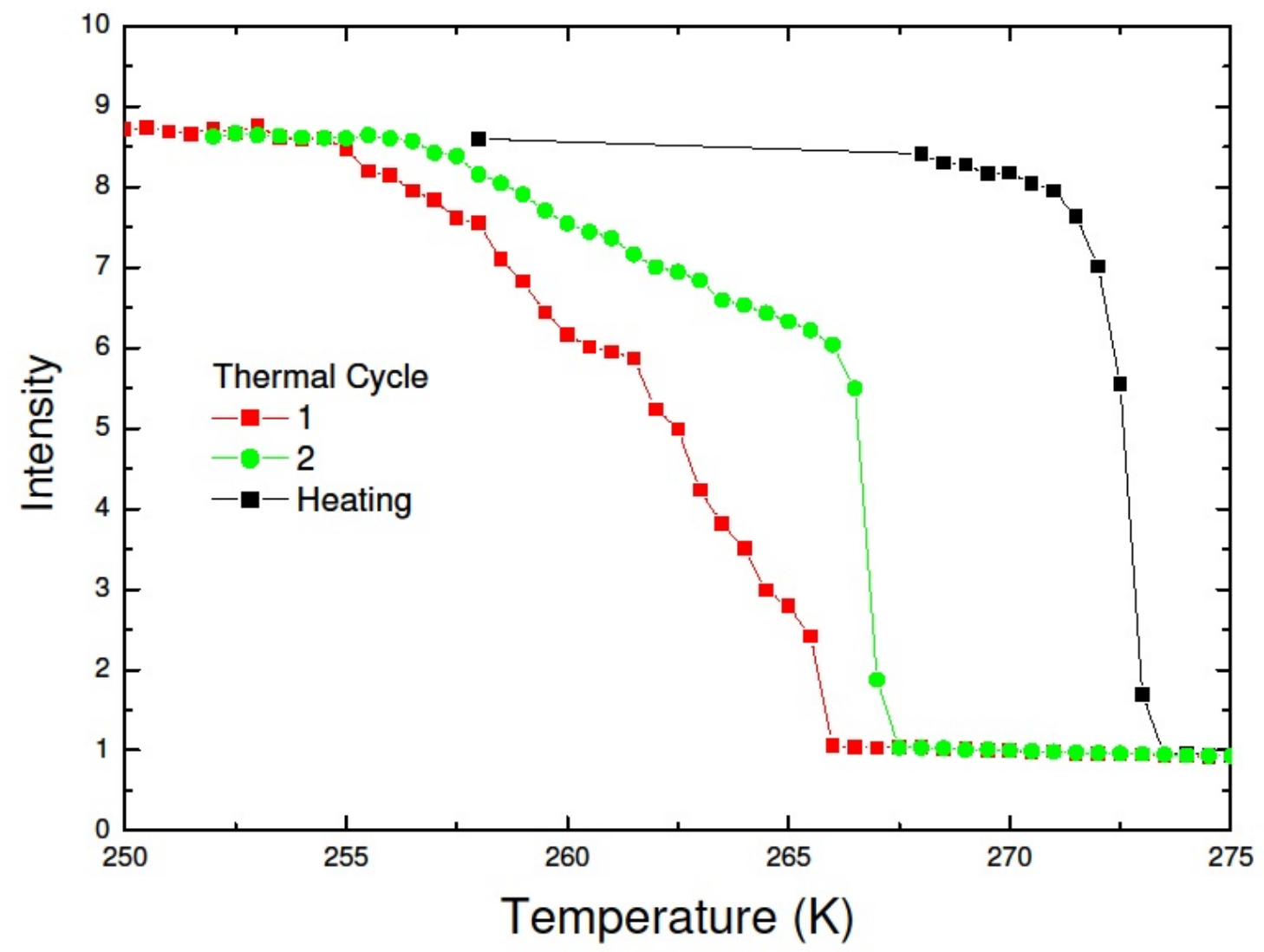

Figure 3.4: Psuedo-elastic intensity on the Wet 1 sample [top curve in Figure 3.2(a)] during two thermal cycles collected on the BASIS instrument at the Spallation Neutron Source at Oak Ridge National Laboratory. Neutrons are binned according to their energy transfer, with the intensity representing any neutron transferring less than $3.5 \mathrm{eV}$ of energy to or from the sample. The intensity is roughly equivalent to the elastic intensity collected on the HFBS. Data points were taken every $0.5 \mathrm{~K}$ and with 60 minutes of counting time during the first (red curve) and second (green curve) cycles. The system is equilibrated for 10 minutes between each point. The heating curve, associated with cycle 1, is representative of both cycles. Between cycles 1 and 2 , the sample is heated to $335 \mathrm{~K}$, above the gel-fluid phase transition, and held for $2 \mathrm{~h}$. The DMPC layer reforms and fills in any defects or holes, such that the second thermal cycle demonstrates a sharp freezing transition at $267 \mathrm{~K}$ followed by a slow continuous freezing upon further cooling consistent with results from the HFBS. 


\subsection{Quasielastic Neutron Scattering from Supported DMPC Membranes}

In order to elucidate the dynamics of the water in this system, full quasielastic spectra were collected on the Backscattering Spectrometer (BASIS) at the Spallation Neutron Source at Oak Ridge National Laboratory in Oak Ridge, TN. Neutrons are produced at this facility by bombarding high-energy protons into a target of liquid mercury, a process called spallation that occurs in a periodic time sequence, thus creating a "birth date" for each neutron. This time-of-flight instrument measures neutron energies by determining the time interval for each neutron pulse to reach the instrument. Use of an 84-m long flight path reduces the uncertainty in the measurement of this time interval. Coupled with the backscattering geometry, BASIS achieves an energy resolution of $3.5 \mu \mathrm{eV}$, corresponding to a timescale of $1 \mathrm{~ns}$ at approximately an order of magnitude higher flux than the HFBS [44].

The largest measurable energy transfer, or dynamic range, of BASIS in the configuration used for this experiment was $\pm 120 \mu \mathrm{eV}$. The previous elastic measurements were collected on HFBS, with a dynamic range of $\pm 17 \mu \mathrm{eV}$ as operated. The larger dynamic range of BASIS is a significant advantage in that it allows measurement of the quasielastic scattering from water over a large range of temperatures that would be inaccessible on the HFBS. 
Physical properties can be extracted from the dynamic structure factor by fitting with a particular lineshape, or scattering law, which may be composed of several components. In the experiment, the dynamic structure factor is convoluted with the resolution function, which is approximately Gaussian in shape. The resolution function is obtained by cooling a sample to $20 \mathrm{~K}$ (such that all motion has slowed to be within the elastic peak) and recording the spectrum.

Because the 2-in diameter sample cans used in these experiments are larger than average samples, it is important to obtain a resolution function measured in this geometry. The quantity $I(Q, \omega)$ is the actual measured intensity. In this case, the spectra were fit using a scattering law composed of two Lorentzians, representing translational diffusion of water and slower lipid dynamics, a delta function in energy transfer corresponding to elastic scattering, and a linear background.

$$
\begin{aligned}
& S_{\text {inc }}(Q, \omega)=\frac{A}{\pi} \frac{\Gamma_{A}}{\omega^{2}+\Gamma_{A}^{2}}+\frac{B}{\pi} \frac{\Gamma_{B}}{\omega^{2}+\Gamma_{B}^{2}}+C \delta\left(\omega-\omega_{0}\right) \\
& I(Q, \omega)=\int_{-\infty}^{\infty} S_{i n c}\left(Q, \omega^{\prime}\right) F_{r e s}\left(Q, \omega-\omega^{\prime}\right) d \omega^{\prime}+m \omega+b
\end{aligned}
$$

It was found that the two Lorentzian components could be separated by their characteristic $Q$ dependence and widths. One component, the "broad" component, increased in width according to $Q^{2}$ at low $Q$, characteristic of translational diffusion of water. The "narrow" component was found to have a $Q$-independent width of 
about 4-6 $\mu \mathrm{eV}$ FWHM. The intensity of the narrow component was also found to be about 15-20 times weaker than the broad component in high hydration samples and identified primarily at high $Q$; thus, identifying this component required focusing on high $Q$ and high temperatures where the broad component had broadened out of the instrument's dynamic range.

Figure 3.5 is a typical spectrum above the freezing step at $268 \mathrm{~K}$ at lower $Q$. Here, it is impossible to identify the narrow component. However, in Figure 3.6 at higher $Q$, the width of the broad component has increased, revealing a weaker narrow component. On cooling, spectra were collected in steps of $0.5 \mathrm{~K}$, counting 60 min per point and were binned in nine $Q$ groups from $Q=0.3$ to $Q=1.9 \AA^{-1}$. 


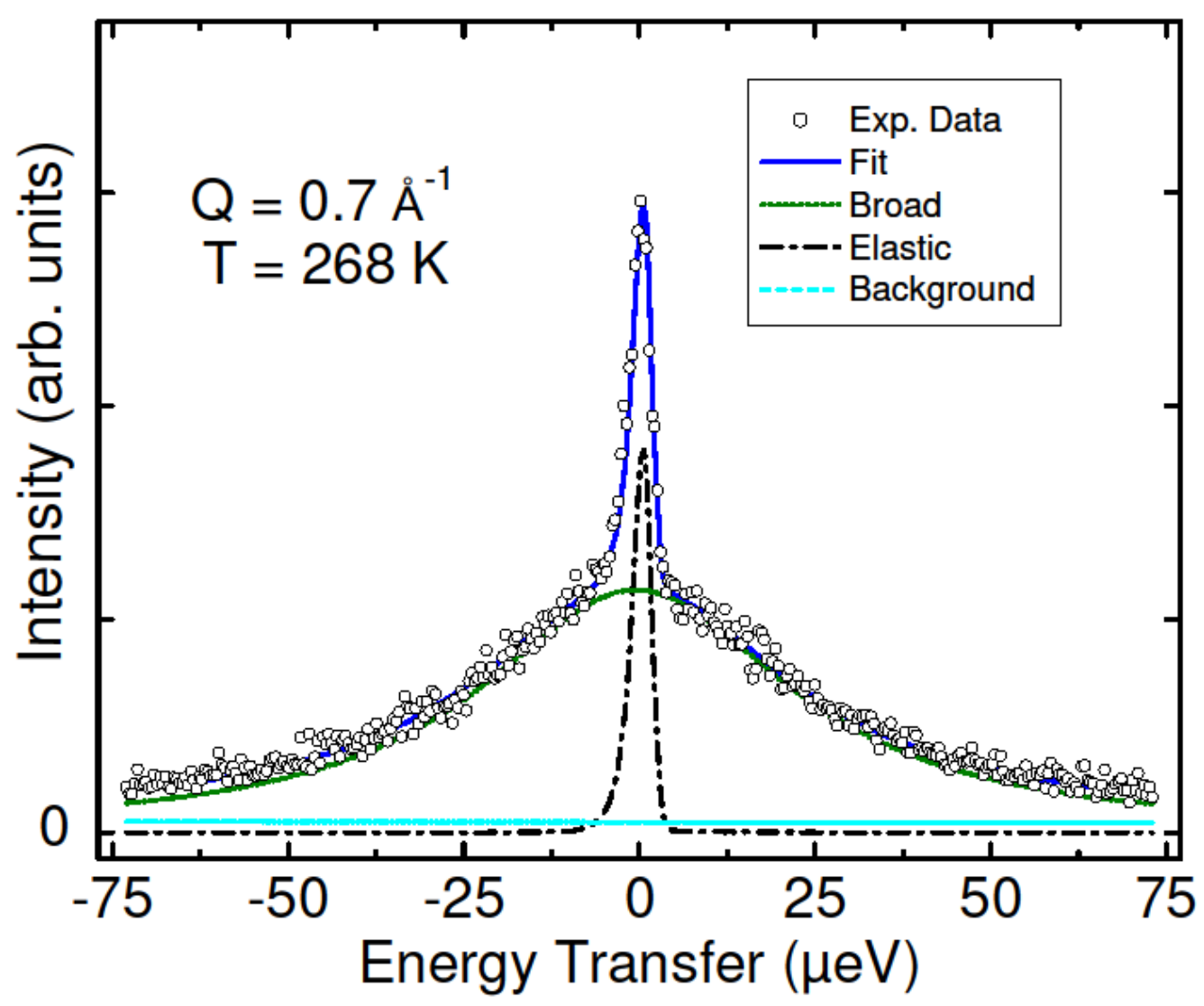

Figure 3.5: Representative spectrum collected on BASIS for the DMPC Wet 1 sample at $Q=0.7 \AA^{-1}$ and $268 \mathrm{~K}$. Data points represent $1 \mathrm{~h}$ of counting time. The spectrum is fit using a scattering law containing a delta function to represent elastic scattering (dash-dot black line), a 'broad' Lorentzian (dashed green line), and a linear background (dashed blue line). The dark blue line represents the total fit to the data after convoluting the scattering law with the instrumental resolution function. The Broad component represents translational diffusion of supercooled bulk water. Collected at low $Q$, this spectrum is fit without an additional 'narrow' Lorentzian component. 


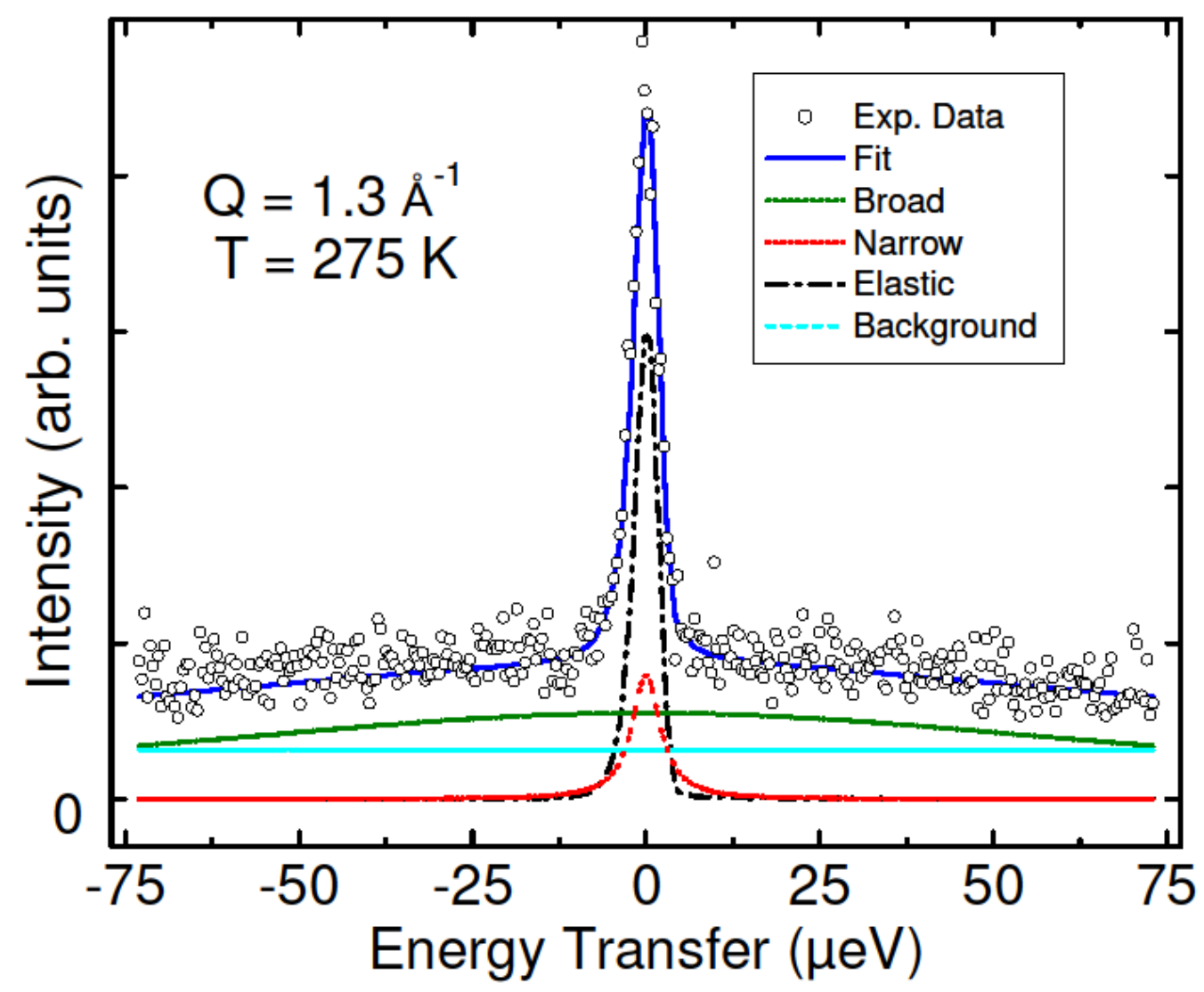

Figure 3.6: Representative spectrum collected on BASIS for the DMPC Wet 1 sample at $Q=1.3 \AA^{-1}$ and $275 \mathrm{~K}$. Data points represent $1 \mathrm{~h}$ of counting time. This spectrum requires an additional 'narrow' component (red dash-dot line) in addition to the broad component (dotted green line). At the higher $Q$ value here, the broad component, representing translational diffusion of water, is wider than the dynamic range of the instrument. The wide and intense background is fit with a larger linear background compared to the spectrum in Figure 3.5. The narrow component, which has a FWHM about $4-6 \mathrm{eV}$ and is about 20 times weaker than the broad component, represents motion of the lipid molecules as well as slow motion of a small number of water molecules bound to the lipid head groups. 


\subsubsection{Identification of Two Types of Water}

The intensity of the broad Lorentzian component and the elastic peak as a function of temperature are shown in Figure 3.7 for the Wet 1 and Dry DMPC samples. The fitting parameters are normalized such that the elastic intensity at $315 \mathrm{~K}$ is unity, which is dominated by elastic scattering from silicon.

In panels (a) and (c), the intensity of the elastic component is shown to compare reasonably well with Figure 3.2, which plots the elastic intensity measured on the HFBS and the second cooling and heating cycle in Figure 3.4 collected on BASIS. There is an abrupt freezing step near $267 \mathrm{~K}$ in the Wet 1 sample followed by a monotonic increase in elastic intensity upon further cooling, while the Dry sample is qualitatively similar but without a freezing step.

The inset in Figure 3.7(c) allows comparison of the hydration level in the Wet 1 and Dry samples. At low temperature, the total increase in the elastic scattering intensity is proportional to the number of immobile hydrogen nuclei. There is approximately a factor of ten higher intensity increase at low temperature in the Wet 1 sample, corresponding to a factor of ten more water. Panels (b) and (d) show the intensity of the broad component, which decreases in intensity on cooling in one-to-one correspondence with an increase in elastic intensity.

The dependence of the elastic intensity on temperature has been described previously in Figure 3.2. In the Wet 1 sample, a large amount of water freezes at 267 


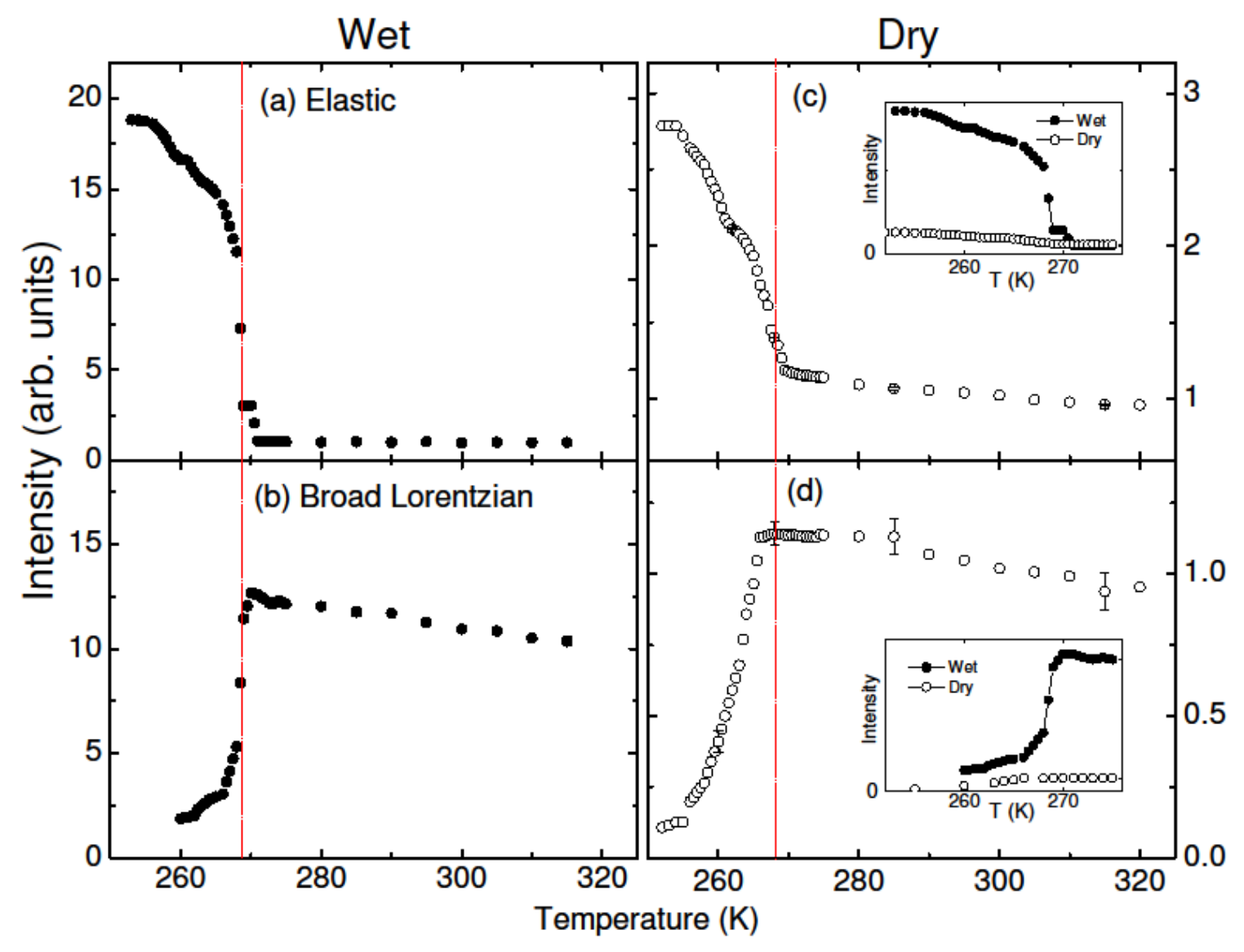

Figure 3.7: Intensities of the elastic [(a) and (c)] and broad [(b) and (d)] components of the quasielastic scattering for the DMPC Wet $1[(\mathrm{a})$ and (b)] and Dry [(c) and (d)] sample during cooling. All values are normalized such that the elastic intensity is unity at $315 \mathrm{~K}$, representing elastic scattering from silicon. The red line at 267 $\mathrm{K}$ denotes the freezing transition of a large amount of water in the Wet 1 sample. For both samples, the sum of the elastic and broad component intensities is approximately conserved which represents the total scattering cross section except the small contribution from the narrow component in Figure 3.11. The water represented by the broad component freezes into the elastic peak below $267 \mathrm{~K}$. Further cooling below $267 \mathrm{~K}$ results in an increase in elastic intensity with a corresponding decrease in the Broad component intensity for both samples. The Dry sample contains about 10 times less water [inset in (c) and (d)]. 
K while a smaller amount of water remains mobile to lower temperatures. The Dry sample does not exhibit the large water freezing step at $267 \mathrm{~K}$; but, nevertheless, shows a continuous increase in intensity as water is frozen on further cooling.

The broad component represents mobile water. In the Wet 1 sample above 267 $\mathrm{K}$, it is much more intense than the elastic component; thus, most of the scattering in this temperature regime originates from mobile water. This component begins to freeze out at $267 \mathrm{~K}$, with an abrupt increase in elastic intensity and a sudden decrease in the broad component intensity at this temperature. Similarly, in the Dry sample above $267 \mathrm{~K}$, the broad component dominates the scattering and begins to decrease in intensity below $267 \mathrm{~K}$; however, the amount of water in the Dry sample is about ten times smaller, and so a large freezing step is not observed.

For both samples, this pattern continues to lower temperature: further increases in elastic intensity and a corresponding decrease in the broad component intensity. The total scattering intensity, the sum of elastic and broad components at a particular temperature, is approximately the total scattering cross section of the sample and is expected to be constant as a function of temperature. This behavior assumes no water is leaving the scattering volume as the sample is cooled.

The slight increase in the broad Lorentzian intensity for both samples as the sample is cooled from 315 to $267 \mathrm{~K}$ can be explained by noting that in this temperature range, as shown in Figure 3.8, the FWHM of this component is just inside the \pm 120 
$\mu \mathrm{eV}$ dynamic range of the instrument. Figure 3.8 shows the width of the broad component for two different $Q$ values as a function of temperature. Note that, for the same temperature, the widths are higher at larger $Q$. For $Q$ values above $1.1 \AA^{-1}$, the FWHM of this component is too large to be reliably fit at temperatures above $267 \mathrm{~K}$. In this case, the width of the scattering is larger than the dynamic range of the instrument and the fitting procedure cannot distinguish between increases in the intensity of the linear background and that of the broad Lorentzian component. Because of this difficulty, the values plotted in panels (b) and (d) of Figure 3.7 are averaged over $Q=0.5,0.7,0.9$, and $1.1 \AA^{-1}$, but not higher $Q$ values. As we lower the temperature, the broad component narrows, effectively lowering the background and increasing the intensity of this component.

Below the freezing step, some of the broad Lorentzian intensity transfers into the elastic peak as more water is frozen; however, the FWHM of this component levels off in the temperature range of 267-262 K just below the freezing step. This plateau in the FWHM vs. temperature is present for both Wet 1 and Dry samples at 267-262 K at two different $Q$ values as shown in Figure 3.8. The water contributing to the broad Lorentzian intensity in the temperature regime between 267 and $262 \mathrm{~K}$ is dynamically different than supercooled bulk water. Because this effect is seen in both the Wet 1 and Dry samples, the latter of which is dominated by water closer to the membrane, we infer that the remaining unfrozen water in the Wet 1 sample below $267 \mathrm{~K}$ interacts 


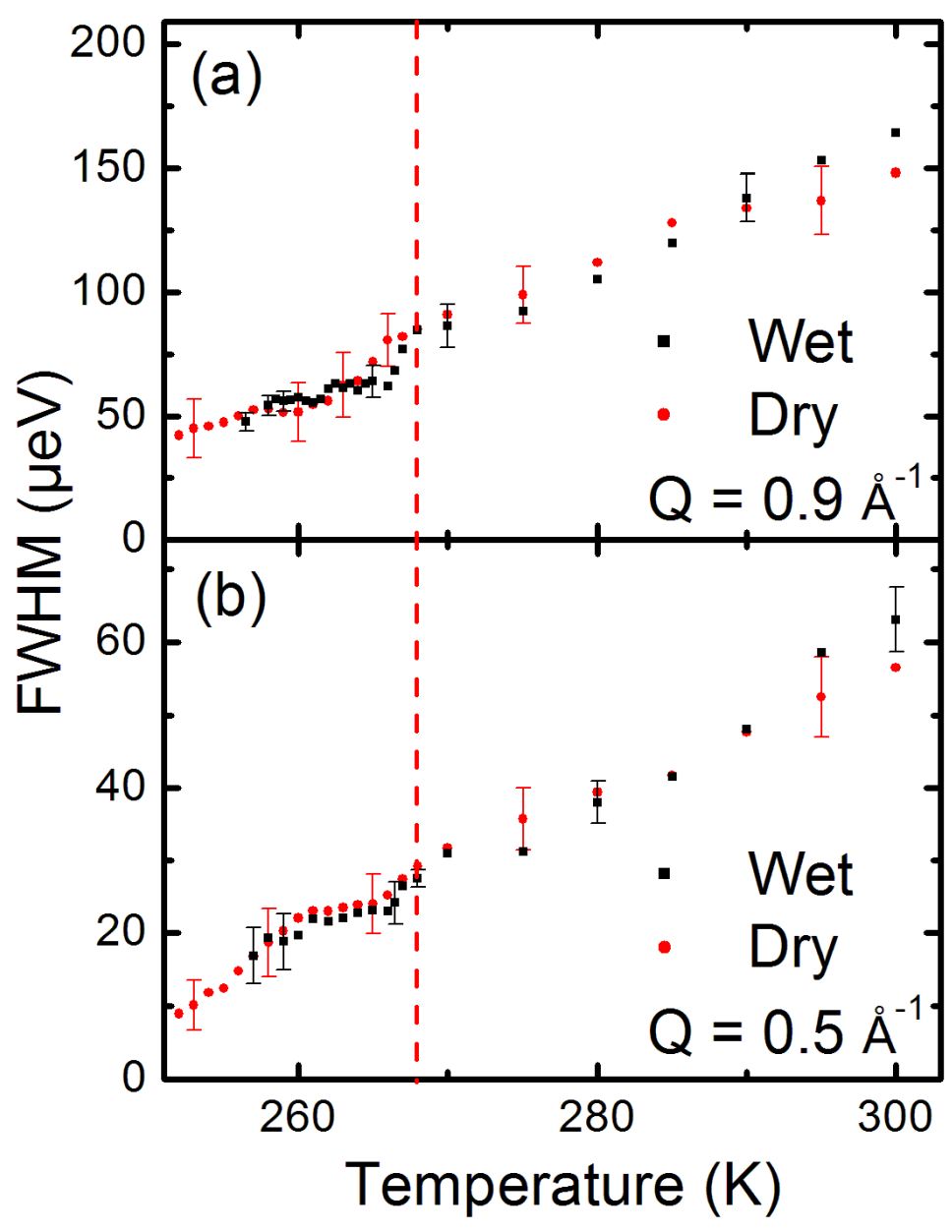

Figure 3.8: Full-width at half-maximum of the broad Lorentzian component as a function of temperature for the Wet 1 (black squares) and Dry (red circles) DMPC samples at two lower $Q$ values. The red dashed line represents the freezing temperature of a large amount of bulk-like water in the Wet 1 sample. For both samples, a plateau region between 260 and $265 \mathrm{~K}$ is observed, indicating different dynamics than bulk supercooled water. The error bars are determined by the method described in Appendix D. 
more strongly with the membrane and has different dynamics than bulk supercooled water.

As the samples are, it becomes more difficult to resolve the broad component due to its intensity decreasing as the water freezes and the corresponding increase in the elastic component intensity. This effect is especially large for the Wet 1 sample for which at $\leq 260 \mathrm{~K}$ almost all of the scattered intensity is within the elastic peak. Fitting parameters can be reasonably obtained down to $258 \mathrm{~K}$ for the Wet 1 sample but can be derived to lower temperatures on the Dry sample due to its smaller elastic peak. At the lowest temperatures $(\leq 260 \mathrm{~K})$ the broad component represents a decreasing amount of mobile water closest to the membrane. The dynamics of this water is slower than bulk supercooled water at the same temperatures.

Based on the FWHM of the broad component for both samples we identify two types of water: a large amount of bulk supercooled water which freezes at $267 \mathrm{~K}$ and a smaller amount of 'confined' water closer to the membrane that diffuses more slowly than bulk supercooled water.

\subsubsection{Diffusion Constants of Water}

We have shown previously that a diffusion constant for water can be extracted from the $Q$-dependence of the width of the broad component in the low $Q$ regime. The half-width at half-maximum is related to the diffusion constant as we saw in Chapter 
1.

$$
\begin{gathered}
\Gamma=D Q^{2} \\
\Gamma=\text { FWHM } / 2
\end{gathered}
$$

For both the Wet 1 and Dry samples, the FWHM of the broad component as a function of $Q^{2}$ is linear at low $Q$. Half the slope of the line that fits this region $\left(Q^{2} \leq\right.$ $0.81 \AA^{-2}$ ) is the diffusion constant. A variety of models can be used to model the higher $Q$ data, including a jump diffusion model [47] or an extended jump diffusion model [50]; however, all models of water diffusion have a limiting case of linearity in $Q^{2}$ for low $Q$ [41]. Therefore, the diffusion constant determined in this way is a model independent measure of the water dynamics.

At higher $Q$ values, the FWHM of the broad component is larger than the \pm 120 $\mu \mathrm{eV}$ dynamic range of BASIS, which makes obtaining reliable fit parameters difficult. Thus, we choose to limit fitting to values of FWHM less than about $100 \mu \mathrm{eV}$. The jump diffusion model has a characteristic leveling off of the FWHM at higher $Q$ as a water molecule moves in a confined volume defined by the transient hydrogen-bonding network formed by its neighbors. Because of these two effects (the limited reliable dynamic range of the instrument and the model-dependent behavior at high $Q$ ), we prefer to focus on the low- $Q$ region of the FWHM vs. $Q$ plot. 


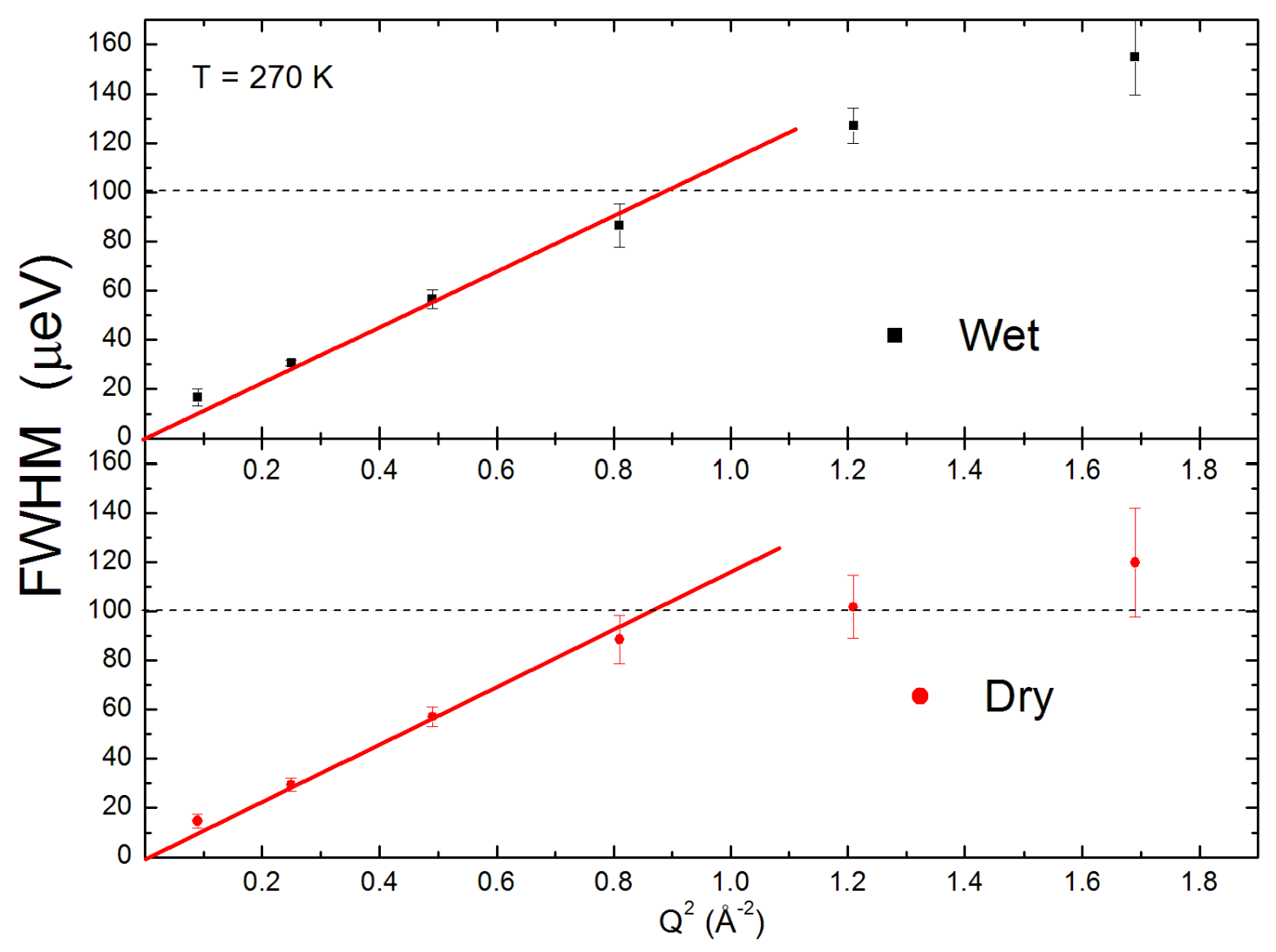

Figure 3.9: Full-width at half-maximum of the broad Lorentzian component as a function of $Q^{2}$ for the Wet 1 and Dry DMPC samples. The dashed black line at 100 $\mu \mathrm{eV}$ represents the dynamic range of BASIS, above which fitting becomes unreliable. The red line represents a weighted linear least-squares fit to the data below $Q^{2}=$ $0.81 \AA^{-2}$ enforced to fit the origin. Half the slope of this line is the diffusion constant, $D$, for translational motion of water according to $\Gamma=D Q^{2}$. The error bars are the standard deviation of the fit as described in Appendix D. 
Two examples of this analysis for the Wet 1 and Dry samples at $270 \mathrm{~K}$ are shown in Figure 3.9. The red line represents a weighted, linear, least-squares fit through the origin for the widths with $Q^{2} \leq 0.81 \AA^{-2}$. At the lowest $Q$ point, the FWHM of the Lorentzian component is $18.2 \mu \mathrm{eV}$ compared to the $3.5 \mu \mathrm{eV} \mathrm{FWHM} \mathrm{of} \mathrm{the}$ resolution function, and there is some deviation from the linear fit in this point. The determination of the width of the broad Lorentzian becomes less reliable the closer it is to the resolution function width. At lower temperatures, the width of the broad component becomes comparable to the resolution of the instrument, reducing the reliability of this analysis. In general, the uncertainty for the Dry data points are larger because of the smaller intensity of the broad component in the Dry sample.

Figure 3.10 shows the diffusion constant as a function of temperature for Wet 1 and Dry samples. First, it is clear that the increased amount of water in the high hydration sample does not affect the diffusion constant as both samples demonstrate identical behavior as a function of temperature. The plateau in the FWHM vs. temperature in Figure 3.8 is seen in the diffusion constant as well, consistent with the remaining unfrozen water having different dynamics than bulk supercooled water. The region between 263-266 K represents a smaller amount of water that diffuses differently than bulk. 


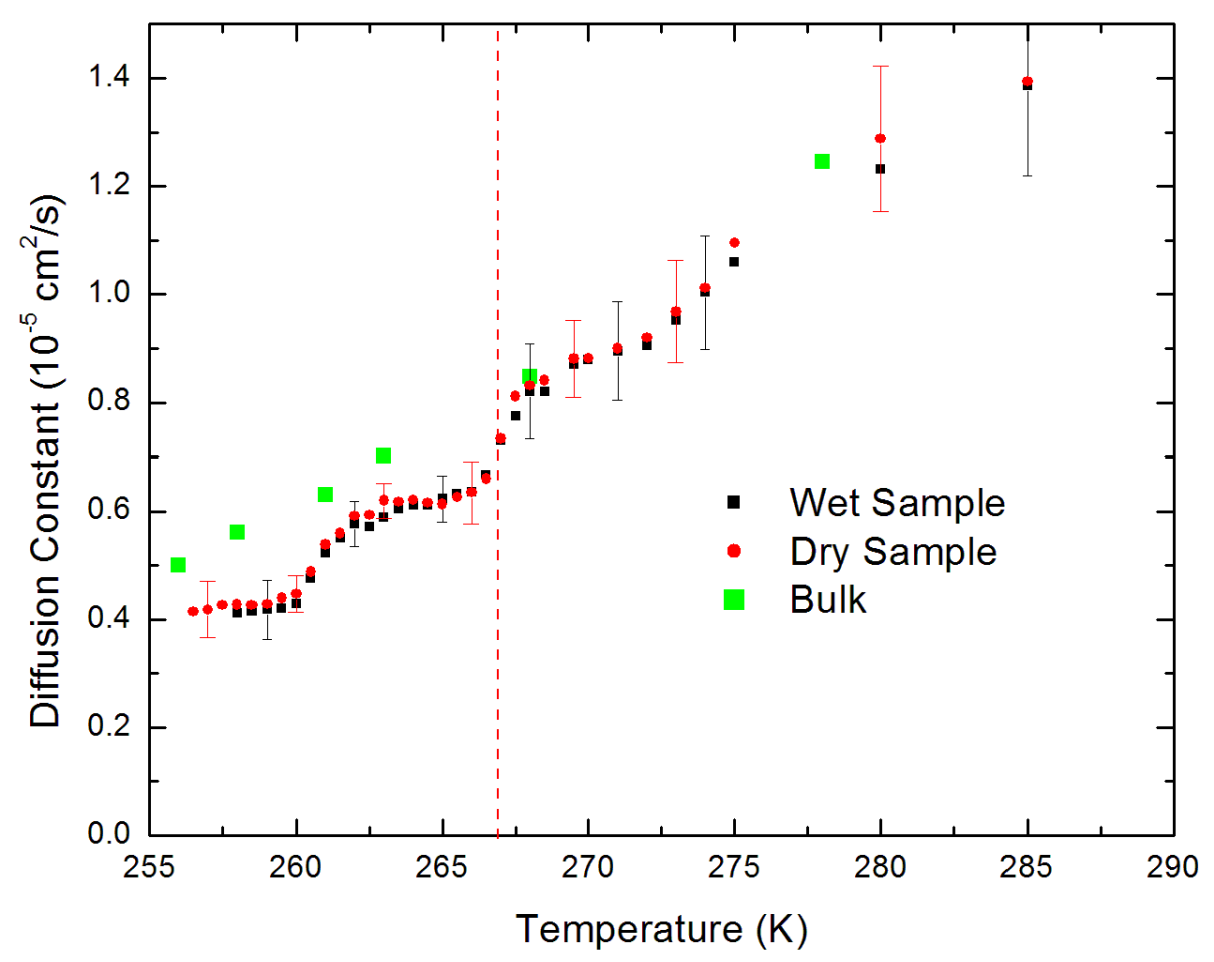

Figure 3.10: Diffusion constants, $D$, calculated as described in the text and illustrated in Figure 3.9 for the Wet 1 (black squares) and Dry (red circles) samples. The diffusion constant is half the slope of the low $Q$ part of the FWHM vs $Q^{2}$ curve. Above $267 \mathrm{~K}$, the diffusion is comparable to literature values for bulk supercooled water measured by Teixeira et al. using quasielastic neutron scattering [47]. Below $267 \mathrm{~K}$, there is a plateau in the dynamics corresponding to the plateau in the width of the quasielastic scattering. The water represented below $267 \mathrm{~K}$ is dynamically different than bulk supercooled water. Further determinations of the diffusion constant below $262 \mathrm{~K}$ are difficult due to the width of quasielastic scattering being comparable to the energy resolution of the instrument. 
The morphology of the interfacial water is unknown in this experiment. If modeled in as a uniform film above the headgroups, the thickness of the mobile water at $266 \mathrm{~K}$ (just below the first step in the diffusion constant) would be $23 \mathrm{~nm}$ thick in the Wet 1 sample and $6.5 \mathrm{~nm}$ thick in the Dry sample. At $260 \mathrm{~K}$, below the second transition in the diffusion constant, the thickness of a film of the remaining mobile water would be $8.4 \mathrm{~nm}$ and $2.8 \mathrm{~nm}$ for the Wet 1 and Dry samples, respectively. Because the amount of water represented in this confined region is small and its morphology is unknown, it is reasonable to consider the results in Figure 3.10 as an effective diffusion constant, representing the average large-length scale behavior of the mobile water as a function of temperature.

\subsubsection{Bound Water Motion - A Third Type of Water}

A second Lorentzian component was needed to fit the spectra at higher $Q$ values accurately. This component was smaller in width (about 10 times narrower) and intensity (approximately 20 times weaker in the Wet 1 sample or about 60 hydrogen nuclei per lipid) than the broad component representing translational diffusion of water molecules.

Shown in Figure 3.11 is the intensity of this narrow component in DMPC membranes as a function of temperature under different conditions of sample hydration and deuteration. The vertical scale is normalized to the elastic intensity at high 
temperature and can be compared directly to the values in Figure 3.7.

The intensity of the narrow component is nearly temperature independent for all deuteration and hydrations conditions with a slight downturn in intensity for the fully protonated (Wet 1) sample below the freezing step. The downturn is attributed to the difficulty in resolving the narrow component, with FWHM on the order of 4-6 $\mu \mathrm{eV}$, from the elastic peak (which is especially intense in the Wet 1 sample) having a FWHM of $3.5 \mu \mathrm{eV}$. Below the vertical red line at $267 \mathrm{~K}$, the broad component has collapsed into the elastic peak.

The spectra analyzed in Figure 3.11 are fit independently and then averaged over the values of $Q=1.3,1.5$, and $1.7 \AA^{-1}$ for which the width of the broad component is much larger than the narrow component. The scattering law used to determine the values in Figure 3.11 is the same as in Equation 3.1 but with the width of the broad component representing translational water diffusion much larger than the dynamic range of the instrument.

The black points represent the Wet 1 sample for which both the water and DMPC membrane are fully protonated. Green points are collected from the Dry sample which was prepared using tail-deuterated $\left(\mathrm{D}_{54}\right)$ lipids. We assume that the water content of the Dry sample, while containing 10 times less water than the Wet 1 sample, is fully hydrated so that intensity of the narrow component is unaffected. In other words, the difference in intensity is only due to the deuteration of the tail 


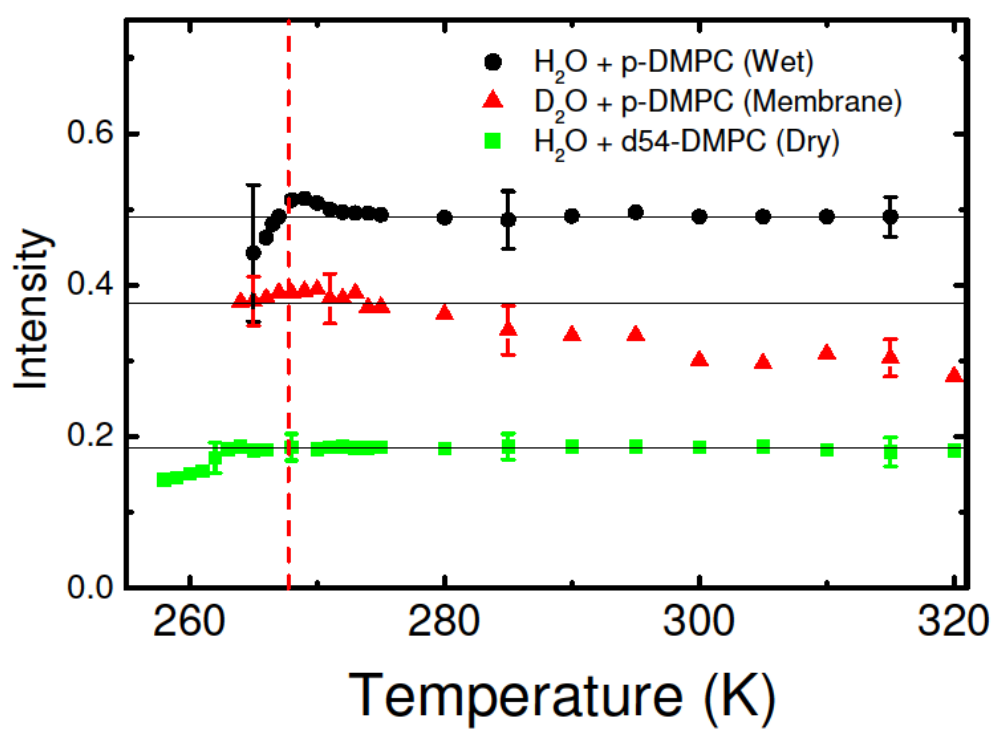

Figure 3.11: Intensity of the nNarrow Lorentzian component as a function of temperature for the Wet 1 (black circles), Dry (green squares), and Membrane (red triangles) DMPC samples. The intensity is fit independently for the $Q$ values 1.3, 1.5, and 1.7 $\AA^{-1}$ and averaged. The intensity is normalized to the elastic intensity at high temperature, representing elastic scattering from silicon. Though the samples are produced with different amounts of water, the relative intensities of the narrow component are explained by the differences in deuteration as it is assumed that each sample has sufficient water to fully hydrate the membrane. The Wet 1 sample, hydrated with $\mathrm{H}_{2} \mathrm{O}$, has the highest intensity. The Membrane sample is hydrated with $\mathrm{D}_{2} \mathrm{O}$ but with protonated lipids. Because the intensity of the Membrane sample is lower than the Wet sample, the narrow component must originate partially from water as well as lipids. Finally, the Dry sample is produced with tail-deuterated lipids. The intensity for this sample is lowest of the three, suggesting that lipid $\mathrm{H}$ atoms contribute most of the motion of the narrow component. Together, the intensity of this component for these three samples suggests that the motion is a combination of lipid motion as well as a small amount of water motion. The black lines represent an average of the data between 265 and $275 \mathrm{~K}$. Below $267 \mathrm{~K}$ (indicated by the vertical red dashed line) the intensity of the elastic peak is much more intense than the narrow component; thus, the reliability of the intensity of the narrow component decreases below $267 \mathrm{~K}$. By averaging values above and below $267 \mathrm{~K}$, the effect of the sudden increase in the elastic peak intensity is reduced. The error bars are determined using the method described in Appendix D. 
groups, which render them invisible to neutrons in this experiment. Finally, the red triangles represent a sample containing protonated lipids but deuterated water (Membrane sample), produced to increase sensitivity to the lipid motion. As is clear, the narrow component is present under each of these three deuteration conditions, implying that the origin of this component must have contributions from hydrogen atoms in water, the lipid tails containing 54 hydrogens, and the lipid head groups containing 18 hydrogens.

Finally, the width of the narrow component as a function of $Q$ is shown in Figure 3.12. For all samples, the intensity of the narrow component decreases with decreasing $Q$ and cannot be reliably resolved below $1.1 \AA^{-1}$. We suggest that this effect may be intrinsic to the motion.

Fundamentally, the dynamic structure factor of our samples represents the probability of a hydrogen nucleus being found in a volume $\Delta V=(2 \pi / Q)^{3}$ after a time $t=\hbar /(2 \pi \Delta E)$. For a bounded motion, the probability of a hydrogen nucleus being found at large distances (small $Q$ ) approaches zero. Scattering from hydrogen nuclei participating in bounded motion will be elastic at low $Q$ values.

Because the narrow component in all samples shows a decrease in intensity with decreasing $Q$ and cannot be resolved at low $Q$, it demonstrates characteristics of bounded motion. 


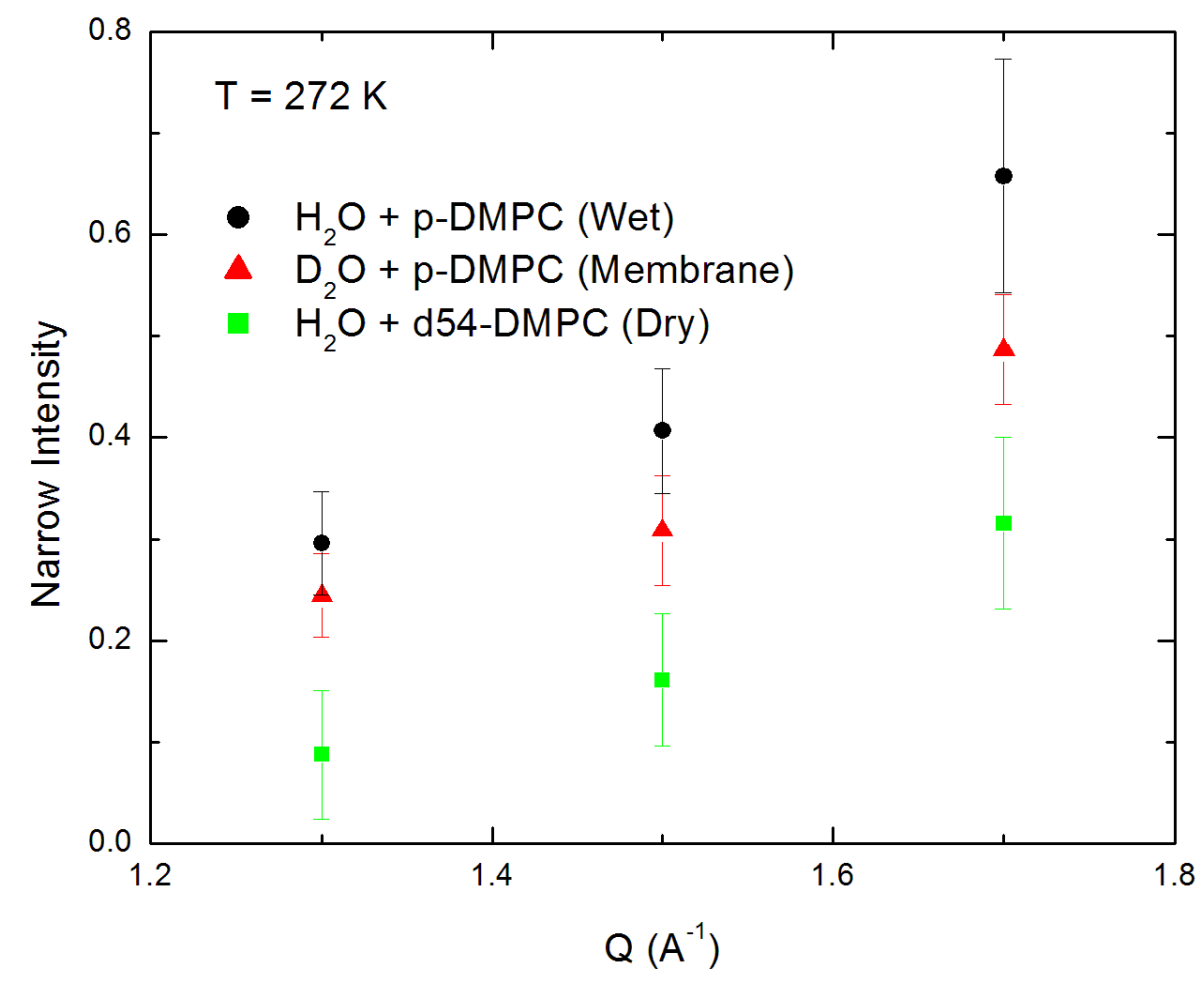

Figure 3.12: Intensity of the narrow Lorentzian component as a function of $Q$ for the Wet 1 (black points), Dry (red points), and Membrane sample (green points) DMPC samples at $272 \mathrm{~K}$. The Wet sample is prepared with $\mathrm{H}_{2} \mathrm{O}$ and protonated lipids. The Dry sample contains lipids with deuterated tails, rendering the tails invisible. The Membrane sample is produced with protonated lipids but with $\mathrm{D}_{2} \mathrm{O}$. For all samples, the intensity decreases with decreasing $Q$. It is not possible to resolve the narrow component reliably below $1.1 \AA^{-1}$. The error bars are determined using the method described in Appendix D. 
We analyzed the intensity of the narrow component to quantify the amount of water participating in the slower motion. Referring to Figure 3.11, which plots the intensity of the narrow component as a function of temperature collected for $Q=$ $1.3,1.5$, and $1.7 \AA^{-1}$ and averaged, we select the values $0.18,0.37$, and 0.48 indicated by the horizontal black lines for the intensity of the deuterated tail and protonated water sample, the protonated tail and deuterated water sample, and the protonated tail and protonated water samples, respectively. These values represent the average intensity of the narrow component in the region between 275 and $265 \mathrm{~K}$. Because the intensity of the elastic peak increases abruptly at $267 \mathrm{~K}$ (affecting the intensity of the narrow component), this region is selected in order to average this effect.

The intensity is proportional to the number of $\mathrm{H}$ nuclei involved in this motion because the contribution from D nuclei is negligible. We consider a single lipid molecule binding an unknown number of water molecules, $n$. There are also 18 hydrogen nuclei in the lipid head groups and 54 in the tails which must be considered. Their contribution is described by $n_{H G}$ and $n_{\text {Tail }}$; both numbers are either 0 or 1 , representing the deuterated and protonated case, respectively. 


$$
\begin{aligned}
& I=\alpha\left(2 n+18 n_{H G}+54 n_{\text {Tail }}\right) \\
& 0.18=\alpha(2 n+18) \quad \text { Dry } \\
& 0.37=\alpha(18+54) \quad \text { Membrane } \\
& 0.48=\alpha(2 n+18+54) \quad \text { Wet } 1 \\
& \therefore n=7.2,8.5,10.7
\end{aligned}
$$

The left-hand side of this series of equations represents the measured intensity, while the right-hand side describes the hydrogens participating in this motion. $\alpha$ is a proportionality constant describing the intensity contribution of one hydrogen nucleus. The series of equations above has three solutions for $n, 7.2,8.5$, and 10.7 water molecules per lipid.

A typical curve of FWHM vs. $Q$ at $272 \mathrm{~K}$ is shown in Figure 3.13 for the three samples above and a 'Superdry' sample which was annealed for three days at 55 ${ }^{\circ} \mathrm{C}$ with no additional water added to the sample can. This sample likely contains just the water strongly bound to the head group. All samples exhibit no significant $Q$ dependence at high $Q$, consistent with bounded motion [41]. With the exception of the Superdry sample, which demonstrates slower dynamics, the more hydrated samples have a comparable FWHM. The 4 - $6 \mu \mathrm{eV}$ energy scale of this motion corresponds 
to a timescale of about 1 ns. Because it is bounded and insensitive to deuteration condition, we infer that the narrow component represents motion of $\mathrm{H}$ in the lipid tails, head groups, as well as the water molecules hydrogen-bonded to the lipid head groups. 


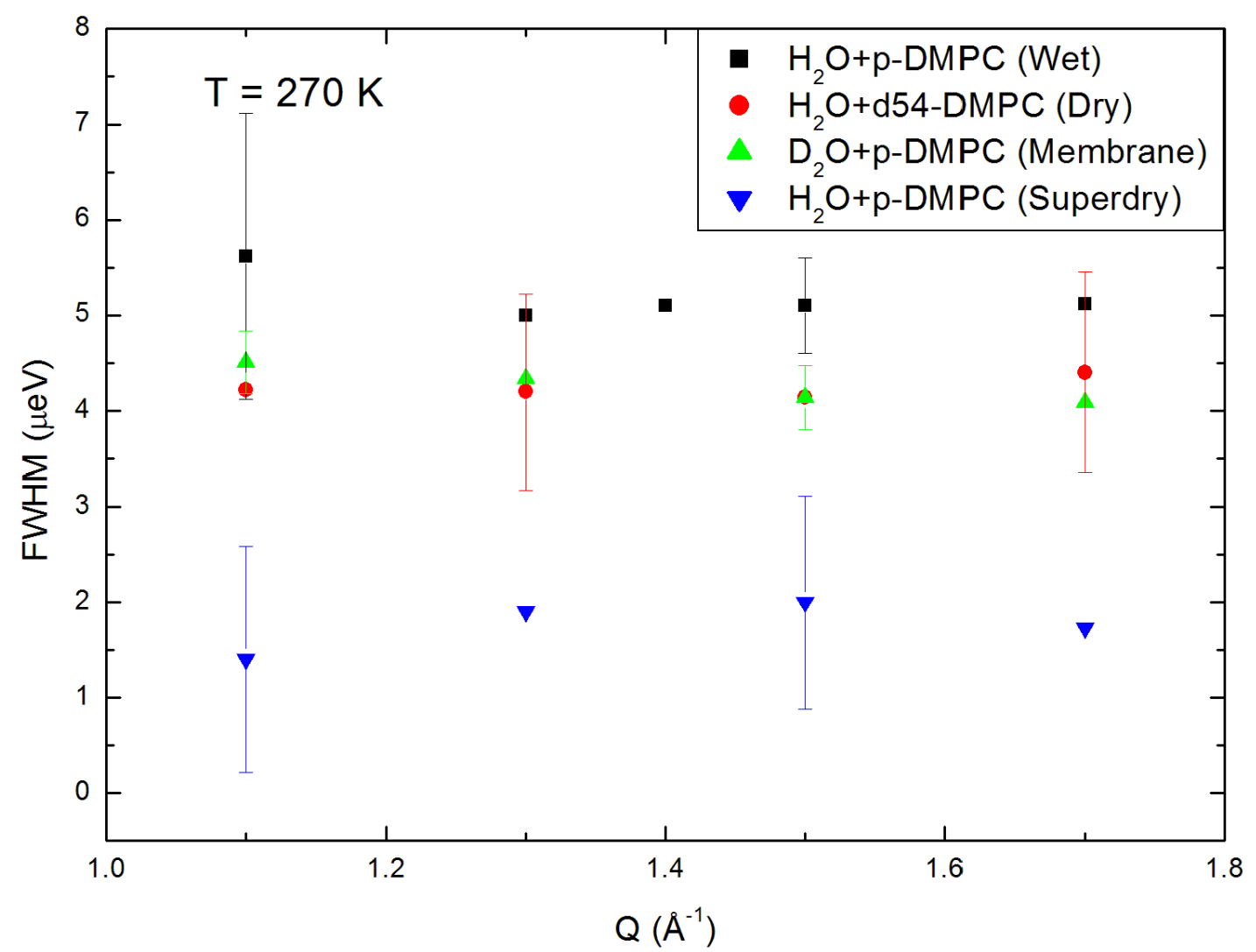

Figure 3.13: Full-width at half-maximum of the narrow Lorentzian component as a function of $Q$ for the Wet 1 (black points), Dry (red points), Membrane sample (green points), and Superdry (blue points) DMPC samples at $270 \mathrm{~K}$. The Wet sample is prepared with $\mathrm{H}_{2} \mathrm{O}$ and protonated lipids. The Dry sample contains lipids with deuterated tails, rendering them invisible. The Membrane sample is prepared with protonated lipids but with $\mathrm{D}_{2} \mathrm{O}$. The top three curves are produced with sufficient water to fully hydrate the membrane; however, the Superdry sample is annealed to remove any bulk water and has no additional droplet of water in its sealed can. Therefore, the Superdry sample contains an amount of water sufficient to saturate the head groups only. It shows slower dynamics, perhaps due to the inability of waters bound to the head group to exchange with the bulk. Despite the differences in deuteration of the Wet 1, Dry, and Membrane samples, their dynamics is similar. All motion is $Q$-independent in this $Q$-range, characteristic of bounded motion. The 4-6 $\mu \mathrm{eV}$ width represents a timescale of about $1 \mathrm{~ns}$. 
Because the Superdry sample was annealed and not rehydrated, it has less water above the membrane. Its low hydration level may explain the relatively slower motion (FWHM about $1 \mu \mathrm{eV}$ ) of the narrow component in this system. Without the presence of sufficient hydration water above the membrane surface, water molecules bound to the head group cannot exchange with a bulk reservoir. Instead, they must jump from site to site between neighboring head groups or remain bound to a single head group. In either case, the elimination or reduction of the head group-to-bulk exchange may slow the dynamics of the remaining water to a nanosecond timescale. Such a site-tosite jump model has been suggested in hydration-dependent QENS studies of DPPC (a DMPC analogue with identical head group) to explain comparably slower motions of low-hydration samples [63].

Figure 3.14 shows the FWHM of the narrow Lorentzian component as a function of temperature for the Wet 1, Dry, and Membrane samples. The width of the narrow component shows only a weak temperature dependence. Below the freezing step of the bulk-like water (indicated by the red dashed line at $267 \mathrm{~K}$ ), it becomes difficult to separate the narrow component from the elastic peak in all samples. The Dry sample, with deuterated tails, demonstrates a slightly narrower FWHM. Possibly, the larger mass of the D atoms results in a slower motion of the lipid molecules' center of mass, resulting in a slower motion of the $\mathrm{H}$ atoms in the head group and bound waters. 


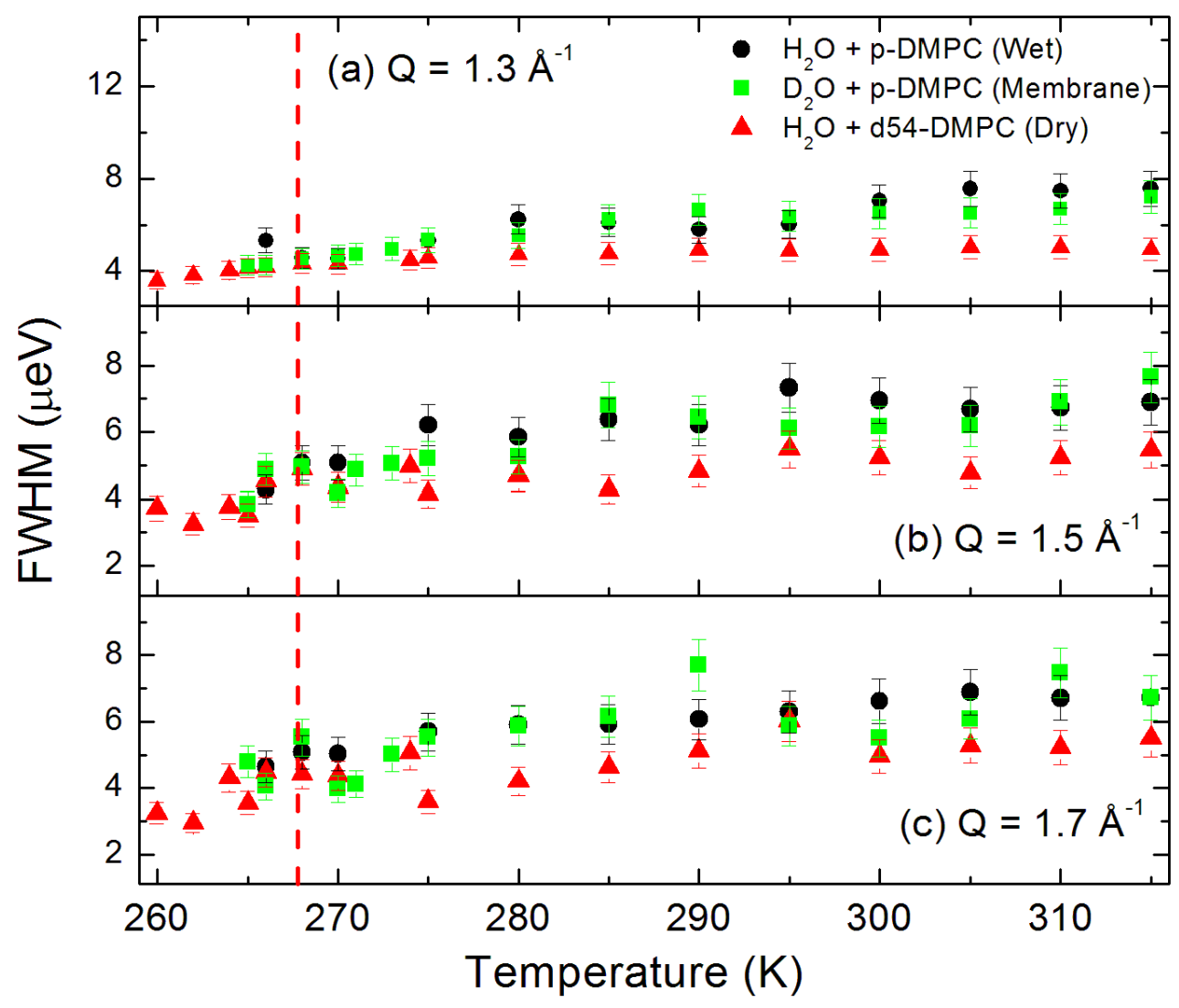

Figure 3.14: Full-width at half-maximum of the narrow Lorentzian component of the quasielastic scattering as a function of temperature for the Wet 1 (black circles), Dry (green squares), and Membrane (red triangles) DMPC samples. The FWHM is calculated for the three most reliable $Q$ values: $1.3 \AA^{-1}$ [panel (a)], $1.5 \AA^{-1}$ [panel (b)], and $1.7 \AA^{-1}$ [panel (c)]. All samples show a $Q$-independent FWHM of 3-5 $\mu \mathrm{eV}$, gradually decreasing with temperature. The Dry sample shows systematically lower FWHM, perhaps due to the slower center of mass motion of the more massive $\mathrm{D}_{54}$ lipids. The 3-5 FWHM width corresponds to timescales of about $1 \mathrm{~ns}$. The vertical dashed red line is placed at the freezing step at $267 \mathrm{~K}$. 
From the intensity and timescale of the narrow Lorentzian component of the quasielastic scattering, the following picture emerges. Based on the characteristic appearance of the narrow component only at high $Q$, this component represents bounded motion. This identification is understood because the probability of a bounded nucleus diffusing to large distances (small $Q$ ) on the timescale of the instrument (which is proportional to the measured intensity) approaches zero. Only at small distances (high $Q$ ) is the probability of finding the nucleus non-zero.

The character of the width and intensity as a function of temperature and $Q$ is nearly identical for all samples. Because selective deuteration was used to isolate either lipid and water motion but motion was measured for all samples, we conclude that both bounded lipid and water motion must contribute to the narrow component. Therefore, we conclude that a small number of water molecules (on the order of 7 - 11), possibly hydrogen bonded to the lipid head groups, move on a time scale of about $1 \mathrm{~ns}$, commensurate with the conformational motion of the lipid tails.

\subsection{Comparison to Molecular Dynamics Simula- tions}

Supporting evidence for the slower, bounded motion may be found in molecular dynamics simulations. Hansen et al. performed a 3.5 ns molecular dynamics simulation 
of a free-standing DMPC membrane at $303 \mathrm{~K}$ [1]. The simulation contained 64 DMPC molecules in each leaflet, for a total of 128 DMPC molecules, as well as 4952 water molecules (38.7 water molecules per lipid). The simulation box was $60 \AA$ in the $x$ direction, $70 \AA$ in the $y$-direction, and $70 \AA$ in the $z$-direction. Atomic coordinates were saved every $0.5 \mathrm{ps}$, giving a total of 7000 time frames. The number density of water molecules (as well as other selected atoms) were then calculated as a function of position from the center of the membrane, averaged over all 7000 frames and is shown in Figure 3.15.

At large distances from the membrane (further than about $25 \AA$ from the center), the density approaches the bulk value. If we integrate the density profile of Figure 3.15 in the $z$-direction from $+23 \AA$ to $-23 \AA$ chosen such that the number density is near bulk, the number of bulk density water molecules in the simulation is 3477 . With 4952 total water molecules, on average 1475 water molecules reside near the head group at less than bulk density. This number corresponds to 11.7 water molecules per lipid. We have suggested in the previous Chapter that the $1 \mathrm{~ns}$, bounded narrow Lorentzian component identified in the quasielastic spectra originates partially from water molecules which are in the vicinity of the head group, numbering 7-11 per lipid.

While the quasielastic scattering cannot directly infer the position of any particular water molecules, the correspondence between the number of water molecules per lipid determined from intensity analysis and the number density profile from the 


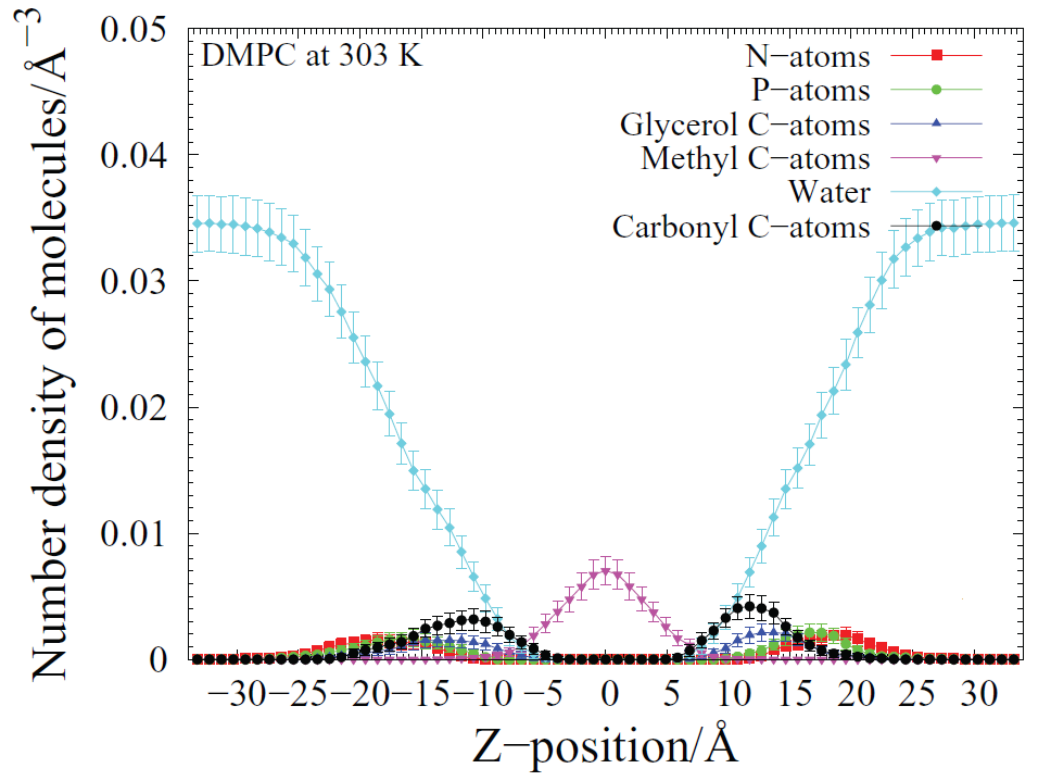

Figure 3.15: Water density profile from molecular dynamics simulation. The number density of water molecules is plotted as a function of distance from the center of the membrane, defined to be $z=0 \AA$, along with the density profile of other selected atoms. The density approaches the bulk value at about $\pm 23 \AA$. The density profile is calculated by averaging over a total of 7000 time frames. From Figure 2(a) from Ref. [1].

molecular dynamics is suggestive.

A second comparison between our experiment and the molecular dynamics simulation relates to the timescale of the narrow spectral component. By tagging individual water molecules near the lipid head groups and following their motion as a function of time, an average residence time of an individual water molecule around the head group can be determined. In this kinetic analysis, water molecules which are within a range of distances $R_{\min }$ to $R_{\max }$ are followed as a function of time. Figure 3.16 plots the number of water molecules which remain in the original region around the selected 


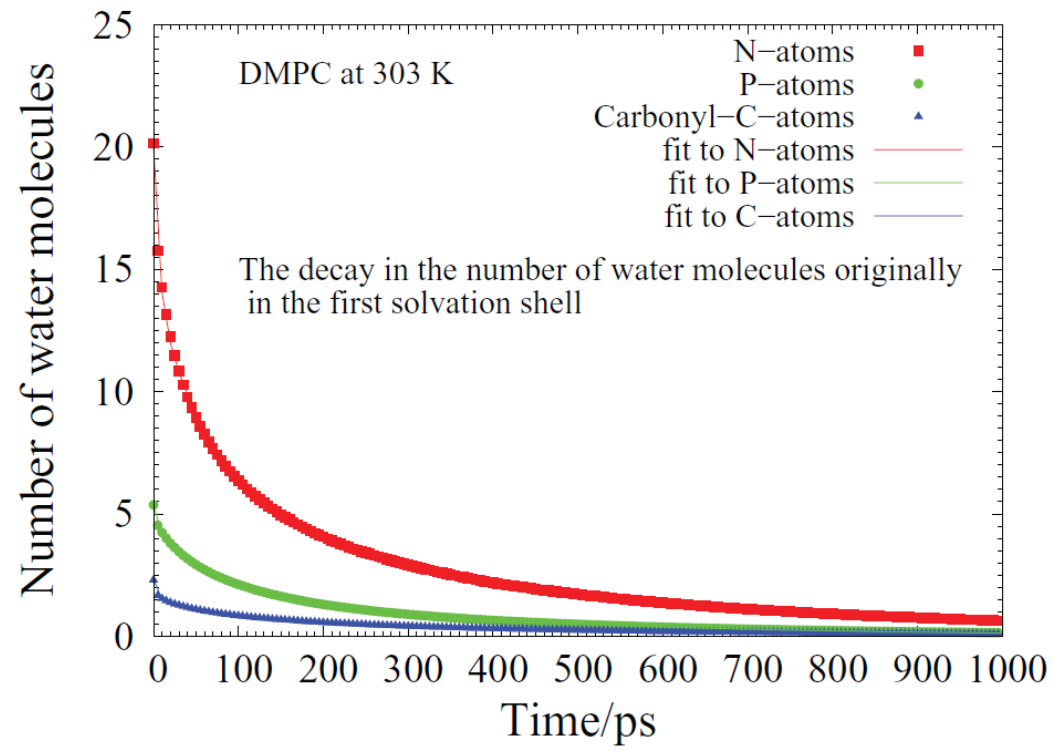

Figure 3.16: Water density profile from molecular dynamics simulation. The number density of water molecules is plotted as a function of distance from the center of the membrane, defined to be $z=0 \AA$, along with the density profile of other selected atoms. The density approaches the bulk value at about $\pm 23 \AA$. The density profile is calculated by averaging over a total of 7000 time frames. From Figure 7 from Ref. [1].

head group atoms over time. The values are averaged over all DMPC molecules and over 900 choices of time origin.

The curves in Figure 3.16 are fit to a series of exponential functions and a time constant is extracted for each exponential term. One term, representing the longest residing and hence strongest bound water molecules, was found to have a time constant of 413 ps. This term represented 8.3 of the original 48 water molecules in the kinetic analysis.

The water density profile suggests that, at any given time step in the simulation, approximately 11 water molecules per lipid reside in the head group region on average. 
A kinetic analysis shows that there are about 8 water molecules per head group bound on a time scale of $400 \mathrm{ps}$. Both of these findings suggest a correspondence with the narrow spectral component in the quasielastic analysis. However, two significant caveats must be mentioned. Hansen et al. perform the simulation at $303 \mathrm{~K}$ in the fluid phase for a free-standing bilayer. While the FWHM was determined from the QENS at $300 \mathrm{~K}$ (in Figure 3.14), AFM results from Chapter 2 indicate that the bilayer is in the gel phase at this temperature. Second, the analysis in the previous Chapter assumes that both leaflets behave identically in terms of water binding and lipid tail motion; and, the presence of the substrate is known to affect the bottom leaflet [30].

\subsection{Summary - Three Types of Water}

In this Chapter, we have described a series of neutron scattering experiments on single-supported bilayer DMPC membranes under different conditions of hydration and deuteration. Previous neutron scattering measurements on DMPC have used multi-lamellar stacks of membranes, containing an unknown number of lipid layers and water. In those measurements, it was impossible to identify the small quantity of water bound to the lipid head groups due to the large and difficult to quantify amount of water between bilayers. By employing a simpler system with just one DMPC bilayer, we are able to identify three types of water: a large amount of bulk-like 
supercooled water, a smaller amount of 'confined' water, and a small number (7 - 11 per lipid head group) of 'bound' water molecules. The confined water extends upwards from the head groups about $40 \mathrm{~nm}$ and diffuses more slowly than supercooled bulk water at the same temperature. The bound water moves on a time scale comparable with the motion of the $\mathrm{H}$ atoms in the lipid molecules (about $1 \mathrm{~ns}$ ).

The Wet 1 sample contains about 10 times more water than the Dry sample; however, the dynamics of the water in each sample, evidenced by the temperature dependence of the FWHM of the broad Lorentzian component in Figure 3.8 and the diffusion constant in Figure 3.10, is nearly identical. The additional amount of water in the Wet sample freezes at $267 \mathrm{~K}$, but does not otherwise affect the dynamics of the remaining confined and bound water.

Below $267 \mathrm{~K}$, the excess bulk-like water is frozen and contributes to the intensity of the elastic peak. The remainder of the broad component represents a second type of water which, based on its intensity, constitutes an amount of water that is equivalent to a film of $40 \mathrm{~nm}$ thickness. At these temperatures, the dynamics of this water is slower than supercooled bulk water. This 'confined' water does not freeze at a single temperature; instead, the monotonic decrease in the broad Lorentzian intensity in both Wet 1 and Dry samples below $267 \mathrm{~K}$ indicates that this component freezes continuously as the temperature is lowered.

We speculate that layers of water furthest from the membrane freeze first, with 
layers closer to the membrane freezing at lower temperature. NMR measurements as a function of temperature have suggested that water molecules in the vicinity of the head group freeze at the lowest temperatures [65]. As such, a layer of unfrozen water exists directly at the head groups. It may be that the unfrozen water directly in the vicinity of the membrane discourages water above it from freezing due to its having a particular orientation. In this case, the formation of the hydrogen bond network required for ice could be inhibited, prolonging freezing to lower temperatures.

Based on the identification of a second, narrow component in the scattering, we infer the existence of a small number of bound water molecules. We infer, based on the $Q$-independence of the quasielastic width of this component and the characteristic appearance of this component only at high $Q$, that these water molecules are spatial bound. The narrow component represents motion of $\mathrm{H}$ in the lipid tails, the head groups, and bound water molecules. The quasielastic width is also nearly independent of deuteration condition, with the exception of the systematically slower motion of the Dry sample which we suggest may be due to the slower center of mass motion of the $\mathrm{D}_{54}$ lipids. This independence implies that the $\mathrm{H}$ in the tails, head groups, and bound waters move on the same time scale, suggesting that their motions are connected, perhaps by the formation of hydrogen bonds between water molecules at the head group. 


\section{Chapter 4}

\section{Neutron Scattering from DMPG Lipid Membranes}

\subsection{Introduction}

As an extension of the work presented for single-supported bilayers of the neutral lipid molecule DMPC, elastic neutron scattering measurements were conducted on samples of supported anionic DMPG membranes. The DMPG molecule is terminated with a neutral glycerol group, giving the molecule a net negative charge that is neutralized in solution. Depending on $\mathrm{pH}$, temperature, and ambient salt concentrations, the supported membrane is described by an ionization number $\alpha_{i o n}$ which ranges from 0 to 1, representing the fraction of DMPG molecules neutralized by counterions [84]. The counterion may be a salt ion such as sodium or potassium; but, under conditions 
of very low lipid concentration, a balance of hydrogen ions in $\mathrm{pH} 7$ water may also provide the predominant counterion. Because of the more complicated charge landscape and the stronger monopole electrostatic interaction that may be present for DMPG membranes, the influence of the membrane on water dynamics is expected to be stronger than in the neutral DMPC case.

In this chapter, we will discuss elastic neutron scattering obtained on singlesupported bilayer DMPG membranes, similar to those found in Chapter 3 on samples of single-supported bilayer DMPC membranes. From these preliminary measurements, qualitative differences between the DMPG and DMPC samples are evident. Furthermore, successive thermal cycles on the same DMPG sample will establish reproducibility of the results.

\subsection{DMPG Neutron Sample Preparation}

Single-supported DMPG membranes were deposited on $\mathrm{SiO}_{2}$-coated $\mathrm{Si}(100)$ substrates as described in Chapter 2. The lipids are prepared by mixing the powder in a chloroform solution and drying overnight to produce a thin film. The film is rehydrated in $15 \mathrm{mM} \mathrm{KCl}, 15 \mathrm{mM} \mathrm{MgCl} 2$ buffer, heated to $70{ }^{\circ} \mathrm{C}$, sonicated for $1 \mathrm{~h}$ or until clear, and filtered through a $100 \mathrm{~nm}$ filter.

Once prepared, the DMPG solution is diluted to a concentration of $15 \mu \mathrm{g} / \mathrm{ml}$. The sample consisted of a stack of 100 silicon wafers that were immersed in the 
Table 4.1: Water content of the DMPG samples

\begin{tabular}{|l|c|c|c|}
\hline Sample Name & Water Volume $(\mu \mathrm{l})$ & Water molecules per Lipid & Film Thickness $(\AA)$ \\
\hline Dry 1 & 12.0 & 318 & 233 \\
\hline Dry 2 & 19.8 & 528 & 386 \\
\hline Wet 1 & 26.7 & 711 & 520 \\
\hline Wet 2 & 36.5 & 972 & 711 \\
\hline
\end{tabular}

solution and incubated for $1 \mathrm{hr}$ at $65{ }^{\circ} \mathrm{C}$ during which time the vesicle fusion process occurred. Upon removal, the surface is hydrophilic; and the remaining buffer solution evaporates. No vigorous rinsing with water is performed subsequent to deposition. Two high hydration samples were then annealed for $72 \mathrm{hr}$ at $65^{\circ} \mathrm{C}$ to remove excess water and sealed in an aluminum sample can with a $120 \mu$ ldroplet of bulk $\mathrm{H}_{2} \mathrm{O}$ as in the case of the DMPC sample. Two low hydration samples were annealed at $65{ }^{\circ} \mathrm{C}$ for 5-10 minutes to remove excess water prior to sealing with no additional water.

The samples listed in Table 4.1 were prepared with protonated lipids and $\mathrm{H}_{2} \mathrm{O}$. They contain a comparable amount of water to the DMPC samples prepared in Chapter 3. The film thickness is defined identically to Table 3.1. If the water is spread uniformly and at bulk density over the membrane surface in a cylinder of 2 in diameter, the height of that cylinder on one side of a wafer would be the film thickness. 


\subsection{Elastic Neutron Scattering from Supported DMPG Membranes}

In the previous chapter, elastic neutron scattering measurements as a function of temperature were described for samples of supported DMPC membranes. These measurements provided insight into the freezing of the membrane-associated water as well as established confidence in the sample homogeneity. In the same way, we used elastic neutron scattering on our DMPG samples to confirm membrane homogeneity independent of AFM and to develop a preliminary picture of water freezing in the vicinity of bilayer membranes of these anionic lipids.

In Figure 4.1, the intensity of elastically scattered neutrons is plotted as a function of temperature for four samples, each having a different level of hydration. All samples show an increase in elastic intensity on cooling down to $200 \mathrm{~K}$. Within the freezing regime (beginning at $260 \mathrm{~K}$ for the highest hydration samples and ending at $200 \mathrm{~K}$ for all samples), there appears to be three regions differing in slope: first, a region of high slope between 260 and $250 \mathrm{~K}$; second, a region of moderate slope between 250 and $230 \mathrm{~K}$; and third, a region of low slope below $230 \mathrm{~K}$ once freezing is complete.

The high slope region at higher temperature (above $250 \mathrm{~K}$ ) appears in all samples but its intensity scales with the total amount of water, indicating that this is the freezing of a relatively homogeneous region of water (evidenced by the narrow width 


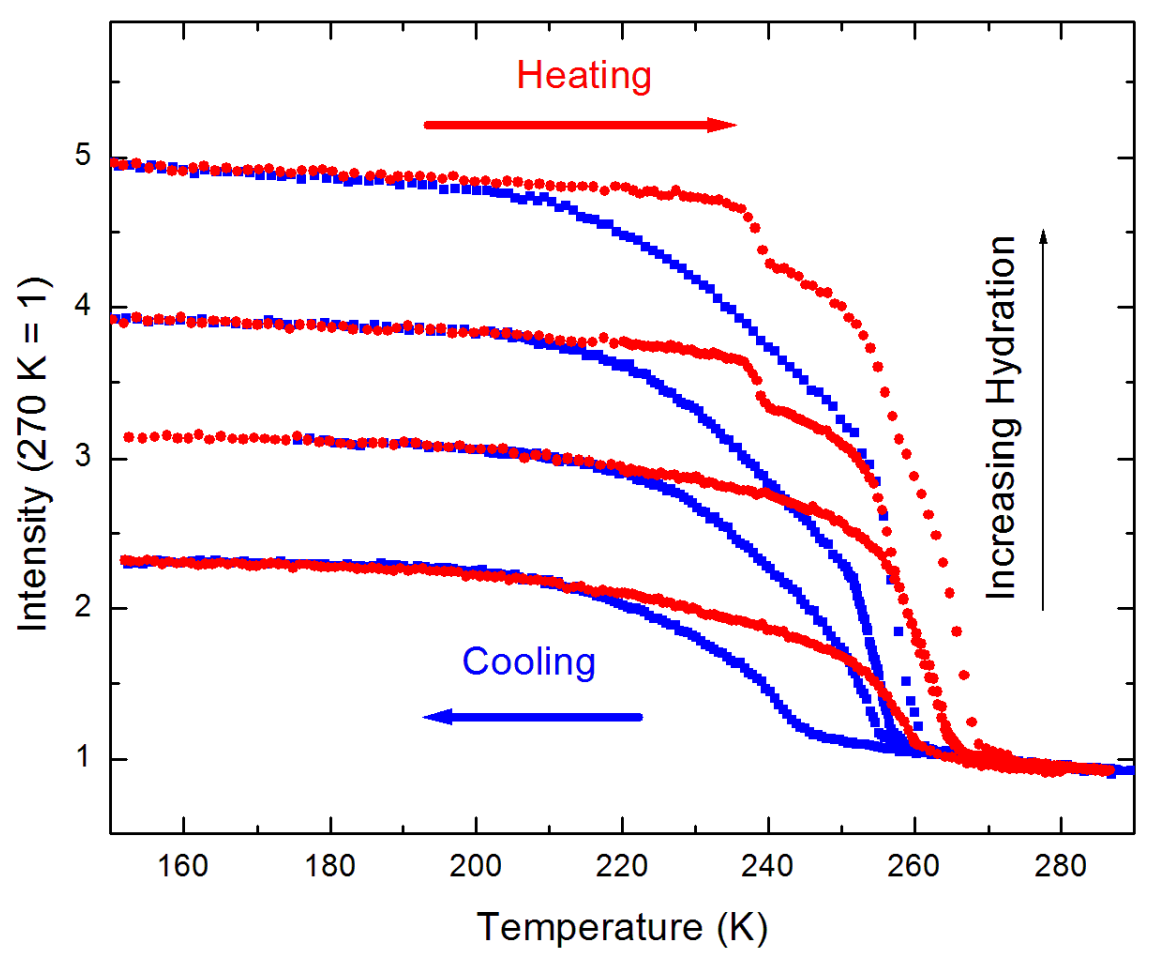

Figure 4.1: Fixed window scans collected on the HFBS on four samples of DMPG each having a different level of hydration. Data points were taken on cooling (blue squares) and heating (red circles). Data is collected for 5 minutes per point with temperature ramps between $0.04 \mathrm{~K} / \mathrm{min}$ and $0.12 \mathrm{~K} / \mathrm{min}$ and are normalized such that the intensity at $270 \mathrm{~K}$ is unity. New features emerge with increasing level of hydration. The two highest hydration samples show a melting substep at $240 \mathrm{~K}$ that does not appear in lower hydration samples. The cooling curves demonstrate three regions of cooling, indicated by the slope of the cooling curve. Most apparent in the highest hydration sample, a region of high slope is seen above $250 \mathrm{~K}$, followed by a region of lower slope between 250 and $230 \mathrm{~K}$, and a final region below $230 \mathrm{~K}$. The cooling and heating curves diverge near $200 \mathrm{~K}$ for all samples, displaying a 10-15 K hysteresis between heating and cooling. 
of this region in temperature) far away from the membrane. The more moderate region of slope (between 250 and $230 \mathrm{~K}$ ) appears in all samples and is therefore freezing of water closer to the membrane. This region is wider in temperature, perhaps indicating that the water represented in this region experiences a more inhomogeneous local environment, consistent with the concept of a strong head group interaction in DMPG membranes.

For DMPG, all samples melt below the bulk melting point of ice $(273 \mathrm{~K})$ as indicated by the temperature at which the lowest level of the elastic intensity is reached. For this reason, we posit that this system does not contain a bulk-like ice configuration. For the two high hydration samples (top two curves), a step-like feature appears on heating at $240 \mathrm{~K}$. Because it appears only in the highest hydration samples, it could represent water furthest away from the membrane; however, its origin is unknown.

In Figure 4.2, successive thermal cycles of low-hydration samples (Dry 1, upper panel; Dry 2, lower panel) are plotted as a function of temperature. Dry 1 was annealed at $353 \mathrm{~K}$ for two hrs between thermal cycles, above the gel-fluid phase transition, while Dry 2 was annealed at $290 \mathrm{~K}$ between cycles. Remarkable agreement is observed between elastic scans taken in successive thermal cycles for both samples. This behavior is consistent with the AFM results from Chapter 2, which found no significant effect on membrane morphology with annealing. 


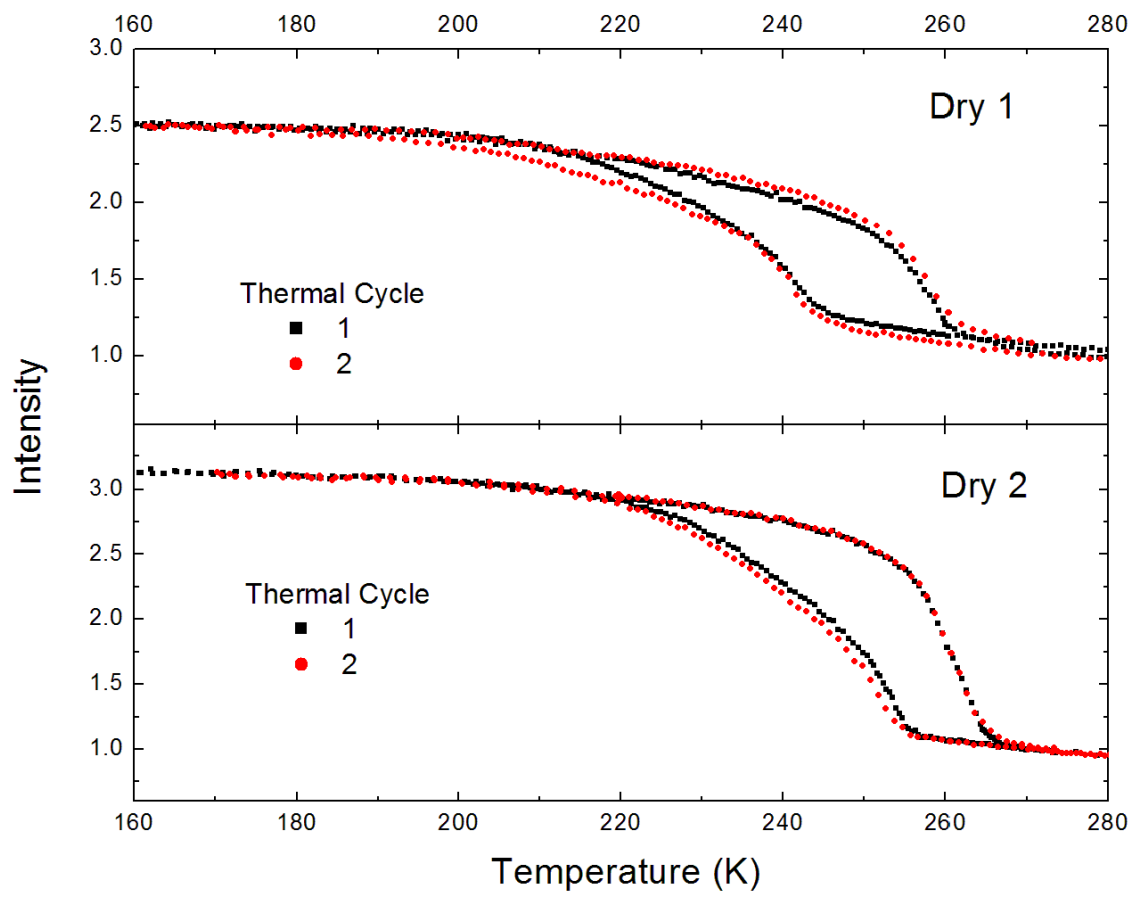

Figure 4.2: Consecutive fixed-window scans on two samples of supported DMPG bilayers. Dry 1 (upper panel) was annealed by initially heating to $353 \mathrm{~K}$ (above the gel-fluid phase transition) and annealed again between thermal cycles. Dry 2 (lower panel) was annealed at $290 \mathrm{~K}$ between cycles. Neither sample shows a strong annealing effect compared to DMPC (Figure 3.4). This behavior is consistent with AFM results which indicate that the DMPG bilayer is less susceptible to annealing. Furthermore, the fact that consecutive thermal cycles reproduce well testifies to the homogeneity and thermal equilibrium of the sample. Data is normalized such that the first cooling is unity at $270 \mathrm{~K}$. Each point represents 5 min of counting time. 


\subsubsection{Three Types of Water in DMPG Samples}

In the previous Chapter, neutron scattering results for the Wet 1 and Dry DMPC samples were presented. As seen clearly by the step-like changes in intensities in Figure 3.7, the Wet 1 DMPC sample contains an excess of bulk-like water above the membrane. However, the dynamics of the water in the two samples is similar despite differences in hydration. This similarity is demonstrated by the independence of the diffusion constant on hydration in Figure 3.10. The width of the quasielastic scattering, both the broad and narrow component, was also independent of hydration (Figures 3.8 and 3.13). In other words, while the Wet 1 DMPC sample contained a large amount of bulk-like water, it was frozen below $267 \mathrm{~K}$ and did not affect the dynamics of the remaining unfrozen water upon further cooling.

This additivity of water is not immediately obvious. In analyzing the narrow spectral component in Figure 3.13, the Superdry DMPC sample displayed slower dynamics than the fully hydrated membranes. Thus, for that sample, the hydration level did affect the dynamics of the mobile water.

For our DMPG samples, however, the elastic scans indicate that the addition of more water does not alter the freezing behavior of water at low temperature. To demonstrate this effect, we consider the slope of the elastic curves as a function of temperature. The slope indicates the quantity of additional water that freezes per degree of cooling. In Figure 4.3, the cooling curves from Figure 4.1 are repeated. We 


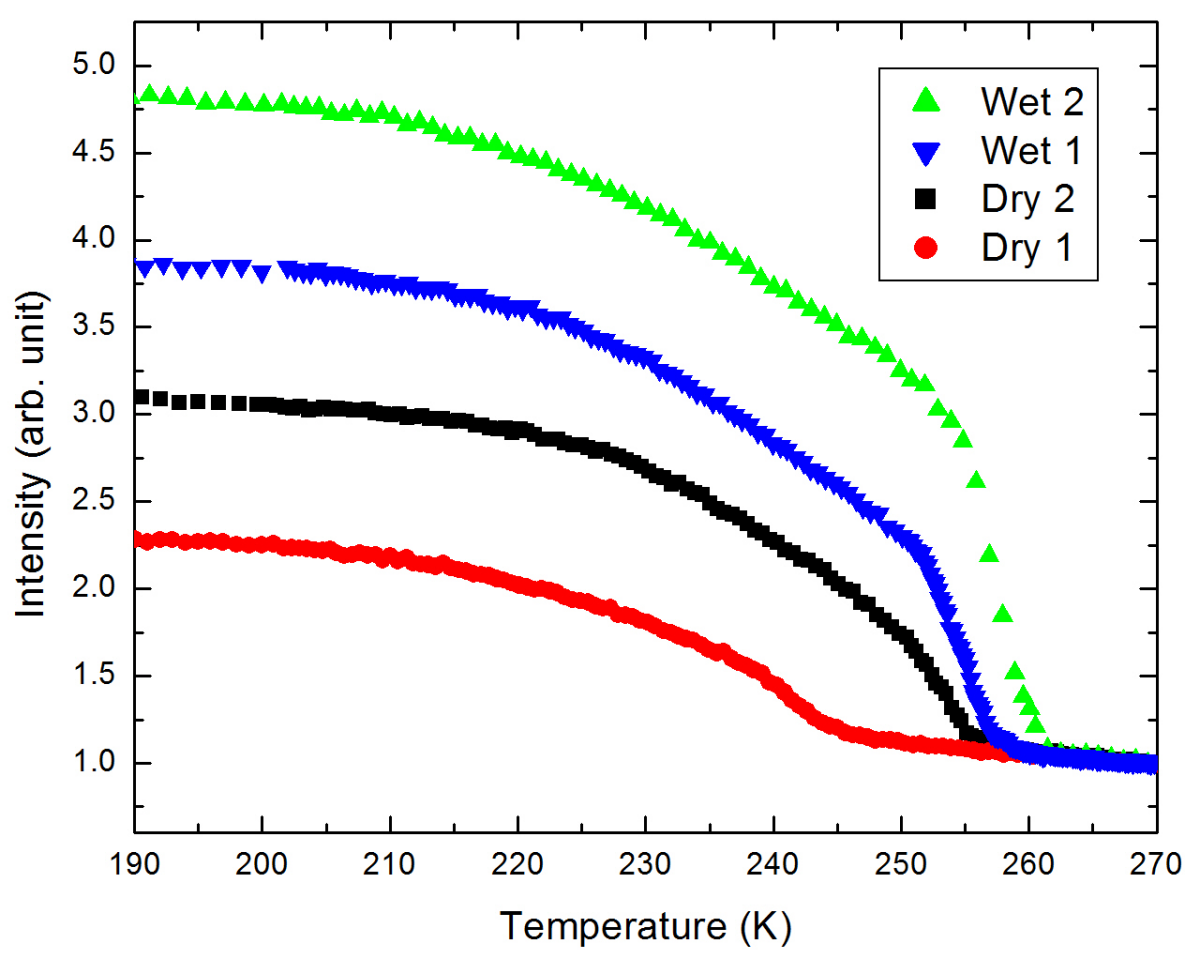

Figure 4.3: Fixed window scans on DMPG samples. The cooling curves presented here are reproduced from Figure 4.1. Data are normalized to unity at $270 \mathrm{~K}$ at which the intensity is dominated by incoherent scattering from silicon. Each point represents 5 min of counting time.

note that the slope of the cooling curves for the Dry 2, Wet 1, and Wet 2 samples between $250 \mathrm{~K}$ and $230 \mathrm{~K}$ are similar. This similarity of the slope of these curves indicates that a comparable quantity of water is freezing in this temperature range. Above $250 \mathrm{~K}$ for the Dry 2, Wet 1, and Wet 2 samples, a region of high slope is also observed in the cooling. The high slope region above $250 \mathrm{~K}$ appears to scale in magnitude with the total amount of water in each sample. 
We identify three regions of interest in the Dry 2 , Wet 1 , and Wet 2 samples shown in Figure 4.4. Region 1 is the high temperature freezing regime in which the increase in intensity in this region scales with the total hydration of the sample, defined above $250 \mathrm{~K}$. The total width of the freezing is spread over only a few degrees; as such, it is similar to the freezing of bulk-like water in the DMPC samples.

Region 2 is a continuous freezing region between 250 and $230 \mathrm{~K}$ which shows similar slope for all samples, indicating that water freezes in a way that does not depend on the total hydration. We note the similarity between Region 2 in the DMPG samples and the "confined", membrane-associated water in the DMPC samples. In the DMPC samples, it was seen that the continuously freezing water can be saturated, meaning additional water enters in a bulk-like freezing step (as in Region 1). If a similar effect is present for the DMPG samples, we may expect that the amount of water in the continuous region will become saturated at higher water content levels.

The increase in elastic intensity across the region is a measure of the amount of water. The elastic intensity increase for the Dry 2, Wet 1, and Wet 2 across Region 2 are $0.96,1.02$, and 0.98 , respectively. The similarity between Dry 2 , Wet 1 , and Wet 2 may suggest that Region 2 becomes saturated at higher total water content.

Region 3 appears at low temperature below $230 \mathrm{~K}$ which may correspond to the freezing of the water molecules closest to the membrane which are the most strongly interacting. Each sample appears to show a smaller amount of water continuously 


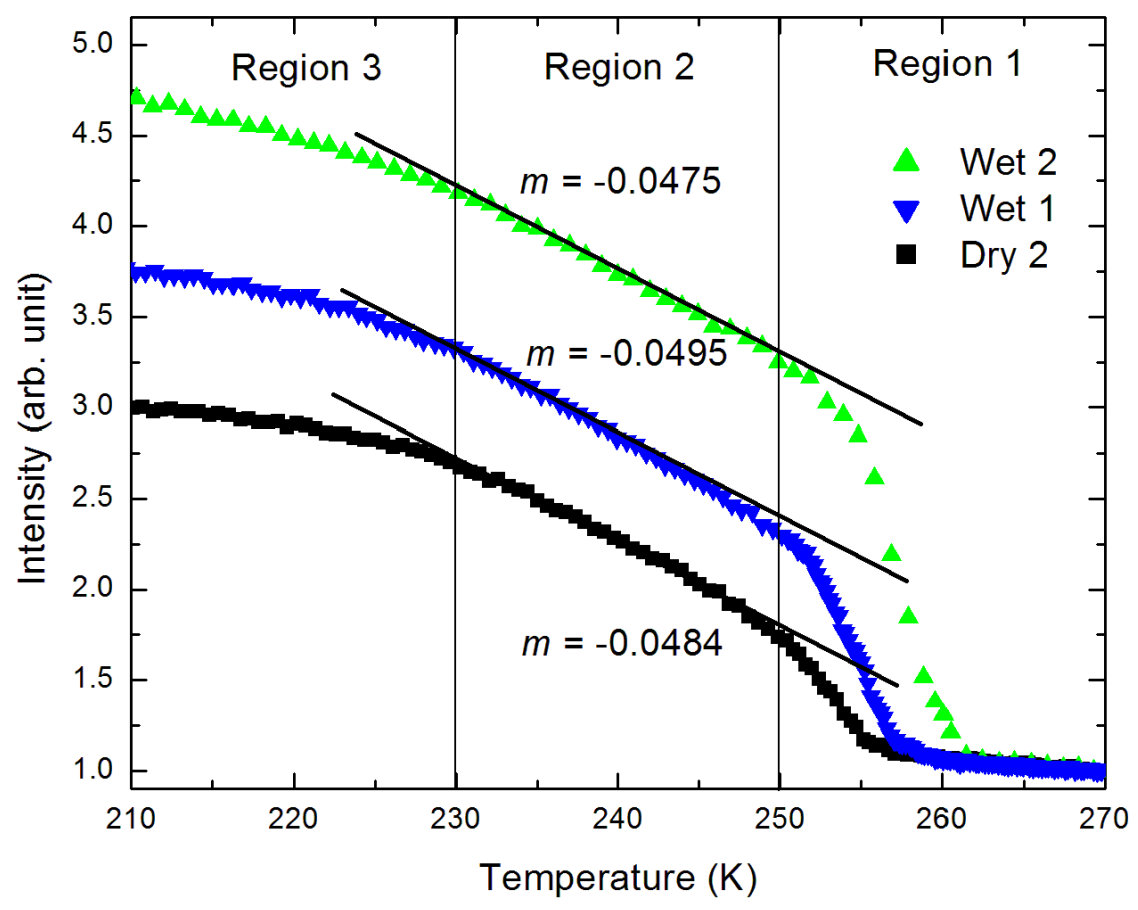

Figure 4.4: Fixed window scans on DMPG samples. Three temperature ranges are identified for the Dry 2, Wet 1, and Wet 2 samples. Region 1 occurs at high temperature. The increment of the increase in intensity scales with the total amount of water; thus, we speculate that this water is similar to the bulk-like water identified in DMPC. Region 2 appears to have the same slope for all samples, indicating that the same amount of water freezes similarly regardless of the total hydration level. Finally, all samples demonstrate a wide, continuous freezing of remaining water in Region 3. The inclined lines all have the same slope and are guides to the eye. Vertical lines demarcate the boundaries of Region 2. We note that the onset temperature of Region 2 is lower for lower hydration samples. 
Table 4.2: Water content of DMPG regions

\begin{tabular}{|l|c|c|c|c|}
\hline Sample Name & Region 1 Film Thickness $(\AA)$ & Region 2 $(\AA)$ & Region 3 $(\AA)$ & Total $(\AA)$ \\
\hline Dry 1 & 21.2 & 127 & 89.5 & 233 \\
\hline Dry 2 & 132 & 178 & 79.9 & 386 \\
\hline Wet 1 & 240 & 189 & 189 & 520 \\
\hline Wet 2 & 409 & 183 & 183 & 711 \\
\hline
\end{tabular}

freezing down to about $200 \mathrm{~K}$. If the water in Region 3 represents water closest to the membrane, we should expect that the total amount of water in this region would be the same, as determined by the increase in elastic intensity between $230 \mathrm{~K}$ and 200 K.

The amount of water in each of these regions for each sample is summarized in Table 4.2. The values in each column represent a uniform film of given thickness, in the same way as for DMPC samples in Table 3.1.

To summarize our expectations, Region 3 water, which represents water closest to the membrane, becomes filled first and does not increase with additional hydration. As we increase the total hydration, Region 2 water then becomes filled until it cannot support more water (becomes saturated). Additional water appears in Region 1, furthest from the membrane. These results are shown in Figure 4.5. The amount of water in Region 3 is independent of the total hydration. Region 2 water increases until it becomes filled. Additional water enters Region 1 which is approximately linear with total hydration. 


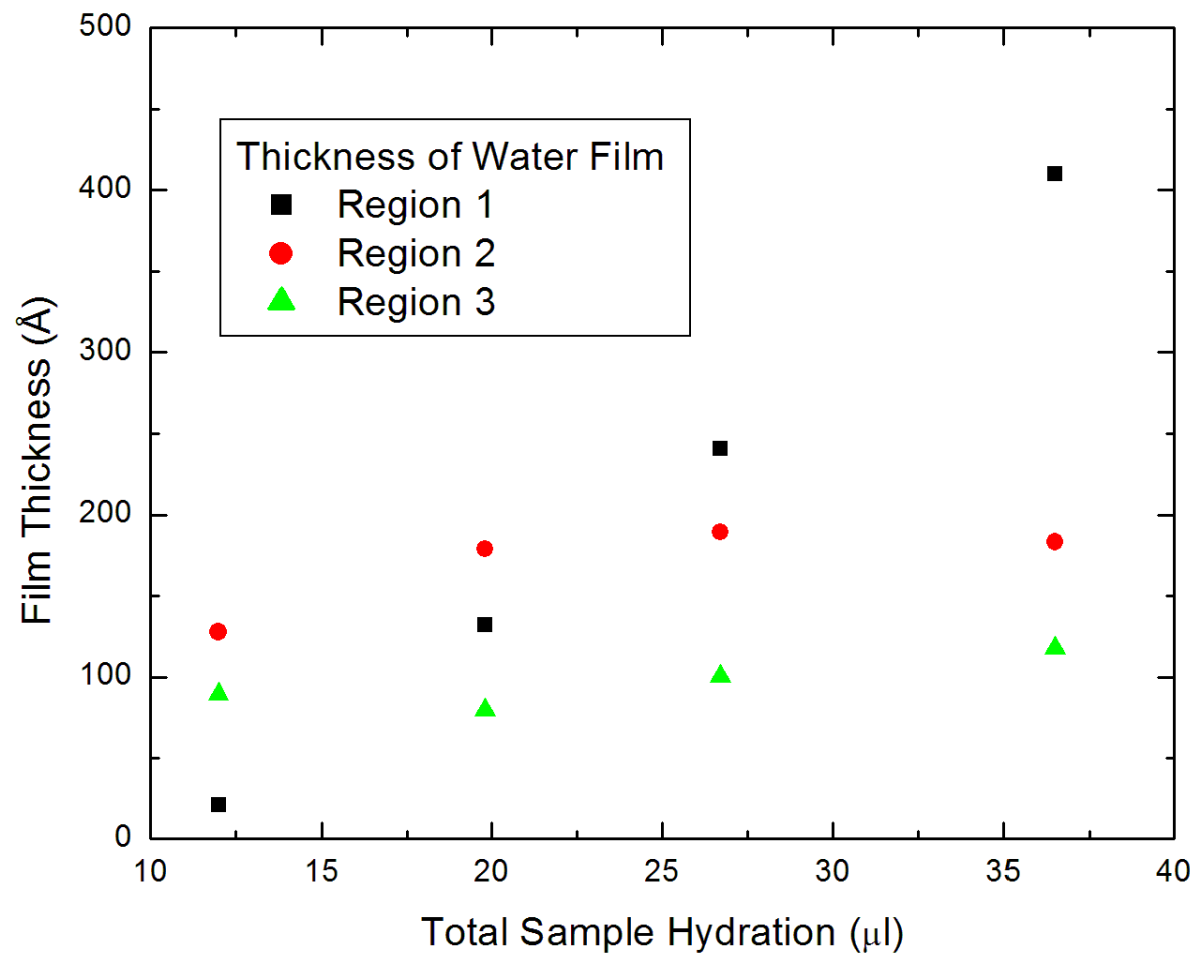

Figure 4.5: Film thickness of water regions in DMPG. The amount of water in Region 1 scales linearly with the total sample hydration. Region 2 water increases for the Dry samples but levels off for Wet samples. The amount of water in Region 3 is independent of the total hydration. 
Therefore, we suggest a picture in which water hydrates the membrane surface first (Region 3) with an amount of water equivalent to about 100 water molecules per lipid. Then, additional water begins to occupy Region 2, further from the membrane. This region can support about twice as much water before becoming saturated. Additional water enters in Region 1 which interacts even less weakly with the bilayer surface and freezes similarly to the bulk-like water in the DMPC samples.

\subsubsection{Melting Behavior of Water in DMPG Membranes}

Ice in all samples melts before the bulk point, $273 \mathrm{~K}$; however, the higher hydration samples completely melts at higher temperatures as seen in Figure 4.6. The lowest

hydration sample, Dry 1, completely melts at $260 \mathrm{~K}$, about $5 \mathrm{~K}$ below the Dry 2 and Wet 1 .

The most striking feature of the heating portion of the elastic scans for DMPG samples is the appearance of a substep at $240 \mathrm{~K}$ for the Wet 1 and Wet 2 samples. We note that the total amount of water that melts in the substep is equivalent to a film of $6.7 \mathrm{~nm}$ in the Wet 1 sample and $8.7 \mathrm{~nm}$ in the Wet 2 sample (about $30 \%$ more in the Wet 2 sample). This difference is approximately the same as the difference in total hydration (36\% more water in the Wet 2 sample). Because it appears only the highest hydration samples, this water may be furthest from the membrane. The origin of the heating substep at $240 \mathrm{~K}$ is unknown. However, because the intensity 


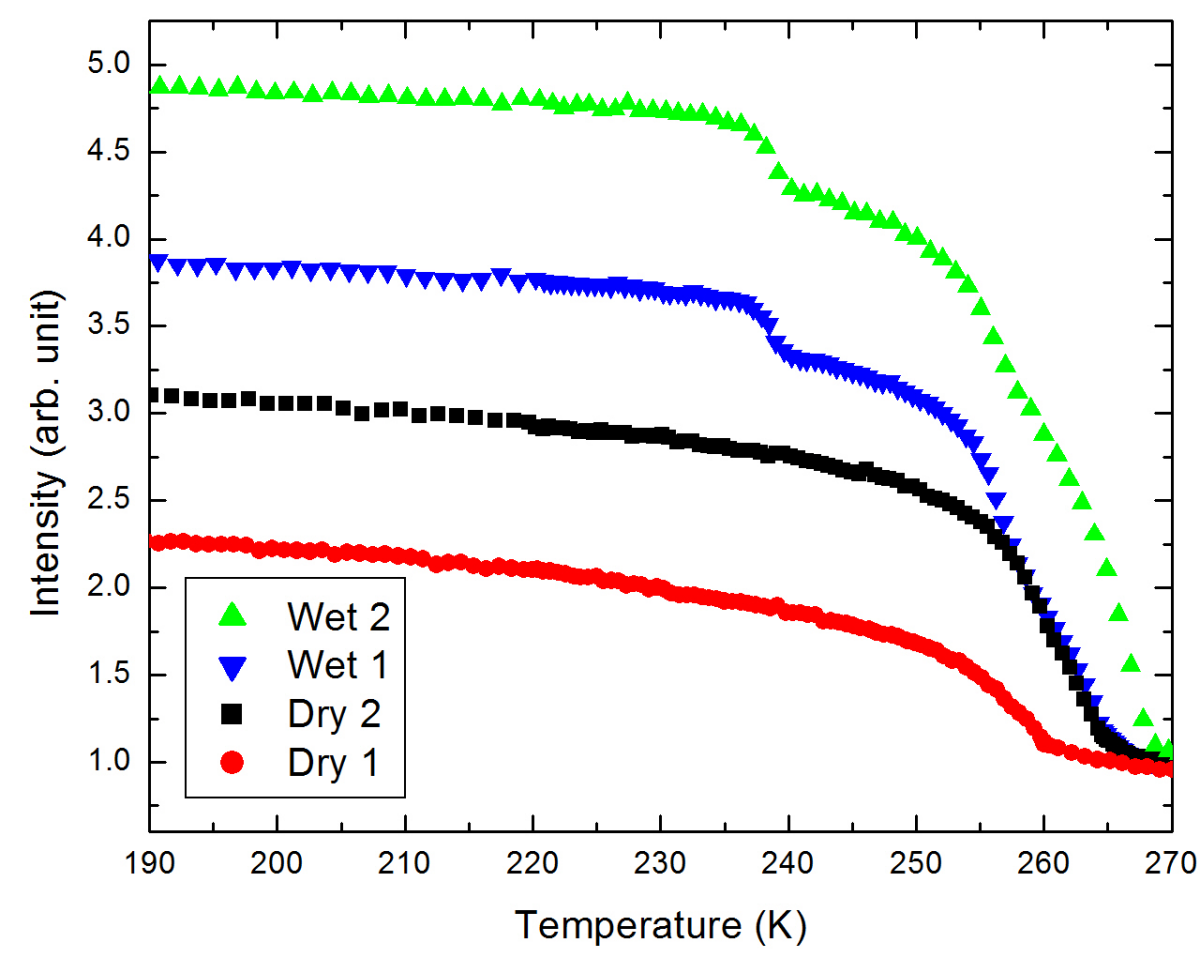

Figure 4.6: Fixed window scans on DMPG samples. The heating curves are reproduced from Figure 4.1. Data are normalized to unity at $270 \mathrm{~K}$. At low temperature, the increment in intensity is proportional to the total amount of water in each sample. Each point represents 5 min of counting time. 
scales with the total hydration and appears only in the highest hydration samples, it is suggestive that it is related to water furthest away from the membrane.

\subsection{Comparison between DMPC and DMPG}

As shown in Figure 3.2, water near the DMPC membrane is completely frozen when cooled to $250 \mathrm{~K}$. On heating, the water melts near the bulk point, indicating that the frozen water is likely in the common bulk, hexagonal crystal structure. In contrast, the DMPG system must be cooled to $200 \mathrm{~K}$ before all motion is frozen on the 4 ns timescale. Furthermore, there is no sharp freezing transition (see Figure 3.2); instead, a continuous freezing region is observed beginning near $255 \mathrm{~K}$ (depending on hydration) and extending to $200 \mathrm{~K}$. The complicated freezing behavior is coupled with the lack of a melting transition at the bulk point. At lower hydrations, the heating curves for DMPG contain a continuous melting region marked by complete melting well below $273 \mathrm{~K}$ (Figure 4.1).

For both DMPC and DMPG samples, the total amount of water can be described by a film on the order of 10-100 $\mathrm{nm}$ in thickness. Our low- and high-hydration DMPC and DMPG samples contain a comparable amount of water, respectively. For example, the Dry DMPC sample contains an amount of water equivalent to a film of $14 \mathrm{~nm}$ thickness, while the equivalent thickness of the Dry 1 DMPG sample is $23 \mathrm{~nm}$. For Wet $1 \mathrm{DMPC}$, the value is $100 \mathrm{~nm}$, while the Wet $2 \mathrm{DMPG}$ is $71 \mathrm{~nm}$. Therefore, 
the significant qualitative differences between the freezing of water near DMPC and DMPG membranes cannot be explained by a difference in total water content.

Another possible explanation for the differences between DMPC and DMPG water freezing behavior is the difference in morphology between the water layers near DMPC and DMPG membranes. While the actual morphology of the water is unknown, the DMPC-coated silicon wafers are hydrophobic (water contact angle $32^{\circ}$ ) upon removal from the incubation solution. Instead of uniformly spreading across the surface, water tends to form droplets. This dewetting effect may be present upon freezing the system as well such that most of the water is in the form of small particles. In such a configuration, most of the water behaves like bulk water with only a small remaining fraction displaying dynamics that differ from bulk supercooled water. In contrast, the freshly prepared DMPG samples are hydrophilic (water contact angle about $23^{\circ}$ ) with water wetting to the surface in a film instead of forming droplets. During cooling, it may be that water near DMPG layers forms a water film over the DMPG head groups of more uniform thickness. Such a configuration may inhibit or prevent the growth of a hexagonal crystal network (the dominant form of ice at standard pressure).

For both DMPC and DMPG samples, water closest to the membrane freezes at the lowest temperatures. This fact is clear because membranes of higher hydration, for both lipids, universally show new features appearing during cooling at higher temper- 
ature and corresponding features on heating also appearing at higher temperatures. In addition, the elastic scans indicate that samples of lower hydration demonstrate freezing behavior at the lowest temperatures. For instance, water in the Dry 1 DMPG sample (Figure 4.1) begins freezing at $245 \mathrm{~K}$, while water in the Wet 2 DMPG sample begins freezing at $260 \mathrm{~K}$. Therefore, we infer that the water closest to the membrane experiences the greatest effect of the surface and freezes at lowest temperatures. Previous NMR measurements have observed mobile water down to $-100{ }^{\circ} \mathrm{C}$, though in amounts below the sensitivity of backscattering spectrometry [88].

In the measurements of the width of the quasielastic scattering (shown in Figure 3.8 ), it is possible to detect water motion down to $252 \mathrm{~K}$ for DMPC and to about $203 \mathrm{~K}$ for DMPG as indicated by the leveling off of the elastic intensity on cooling. Because the water freezing at lowest temperature is closest to the membrane and the PG head group is negatively charged, it is plausible that water remains mobile in the DMPG system to lower temperatures. 


\section{Appendix A}

\section{Quantifying the Amount of Water in Neutron Scattering Samples of Bilayer Lipid Membranes}

In order to build a model of water dynamics it is necessary to determine the total amount of water in each sample. Calculating the absolute amount of water must rely on an independent calibration for which a standard sample of known hydrogen content is required. A previously measured sample containing C32 alkane molecules contains a similar amount of hydrogen and silicon wafers and has the advantage of not containing any water molecules.

Quasielastic neutron scattering scans similar to those taken for DMPC in Chapter 3 were collected on the BASIS instrument for a sample of 10 monolayers of alkanes. The spectra were fit using a scattering law similar to the one used to model water dynamics in DMPC membranes: 


$$
\begin{gathered}
S_{i n c}(Q, \omega, T)=A \delta(\omega)+B L\left(\Gamma_{1}, \omega\right)+C L\left(\Gamma_{2}, \omega\right) \\
L(\Gamma, \omega)=\frac{1}{\pi} \frac{\Gamma}{\Gamma^{2}+\omega^{2}} .
\end{gathered}
$$

The scattering law contained two Lorentzians, characterized by their half-width at half-maximum (HWHM) $\Gamma_{1}$ and $\Gamma_{2}$ and a delta function in energy representing elastic scattering. The intensities $A, B$, and $C$ can be compared directly as $\int_{-\infty}^{\infty} \delta(\omega) d \omega=$ $\int_{-\infty}^{\infty} L(\Gamma, \omega) d \omega=1$. Furthermore, $A, B, C, \Gamma_{1}$, and $\Gamma_{2}$ are functions of temperature and $Q$. Lorentzian components were distinguished by their characteristic timescales into a "slow" (represented by $B$ ) and a "fast" motion $(C)$. The slow motion was on the order of 1 ns and was identified with conformational changes of the alkane tails. The faster motion represented a uniaxial rotation of the alkane molecule about its long axis.

The total number of hydrogen atoms in a sample of 10 monolayers of alkane can be calculated by knowing the crystal structure of adsorbed alkane films. The C32 system is described by a two-dimensional rectangular lattice of sides $a=7.57 \AA$ and $b=4.98 \AA$ with two molecules per unit cell [89]. It is straightforward to calculate the number of hydrogens (approximately $1.1 \times 10^{17}$ alkane molecules on one side of a 2 in diameter Si wafer). The uncertainty in the number of hydrogen nuclei in this system is due primarily to the uncertainty in determining the number of layers of C32, which is determined by measuring the volume of the adsorbed films using AFM. Knowing the total number of hydrogen nuclei is important because the following relationship holds 


$$
\int_{0}^{4 \pi} \int_{-\infty}^{\infty} S_{i n c}(Q, \omega, T) d \omega d \Omega=\alpha \sigma_{t o t}
$$

The above equation states that the total scattering intensity is proportional to the total cross section of the system. The right hand side is constant as a function of temperature since the scattering volume is constant throughout the experiment. To confirm this, the parameters $A, B$, and $C$ are plotted as a function of temperature in Figure A.1 from Ref. [90]. The values are summed over all $Q$ values in the experimental spectra $\left(0.3 \AA^{-1}-1.9 \AA^{-1}\right)$.

Because the right hand side of Eq. A.3 is constant, the left hand side is constant for any value of $\mathrm{T}$, for instance

$$
\int_{0}^{4 \pi} \int_{-\infty}^{\infty} S_{i n c}\left(Q, \omega, T_{1}\right) d \omega d \Omega=\int_{0}^{4 \pi} \int_{-\infty}^{\infty} S_{i n c}\left(Q, \omega, T_{2}\right) d \omega d \Omega
$$

in particular,

$$
\int_{0}^{4 \pi} A\left(Q, T_{1}\right)+B\left(Q, T_{1}\right)+C\left(Q, T_{1}\right) d \Omega=\int_{0}^{4 \pi} A\left(Q, T_{2}\right)+B\left(Q, T_{2}\right)+C\left(Q, T_{2}\right) d \Omega
$$

after calculating the energy integral to eliminate the Lorentzian and delta function terms. At higher temperature, all $\mathrm{H}$ atoms will move on a time scale faster than $4 \mathrm{ns,}$ such that the only remaining contribution to the elastic intensity is from the silicon substrate. This assumption is consistent with the high-temperature data in Figure A.1. At high temperature $(346.5 \mathrm{~K})$, the elastic, broad, and narrow components are all independent of temperature, implying that the limiting value of elastic intensity 


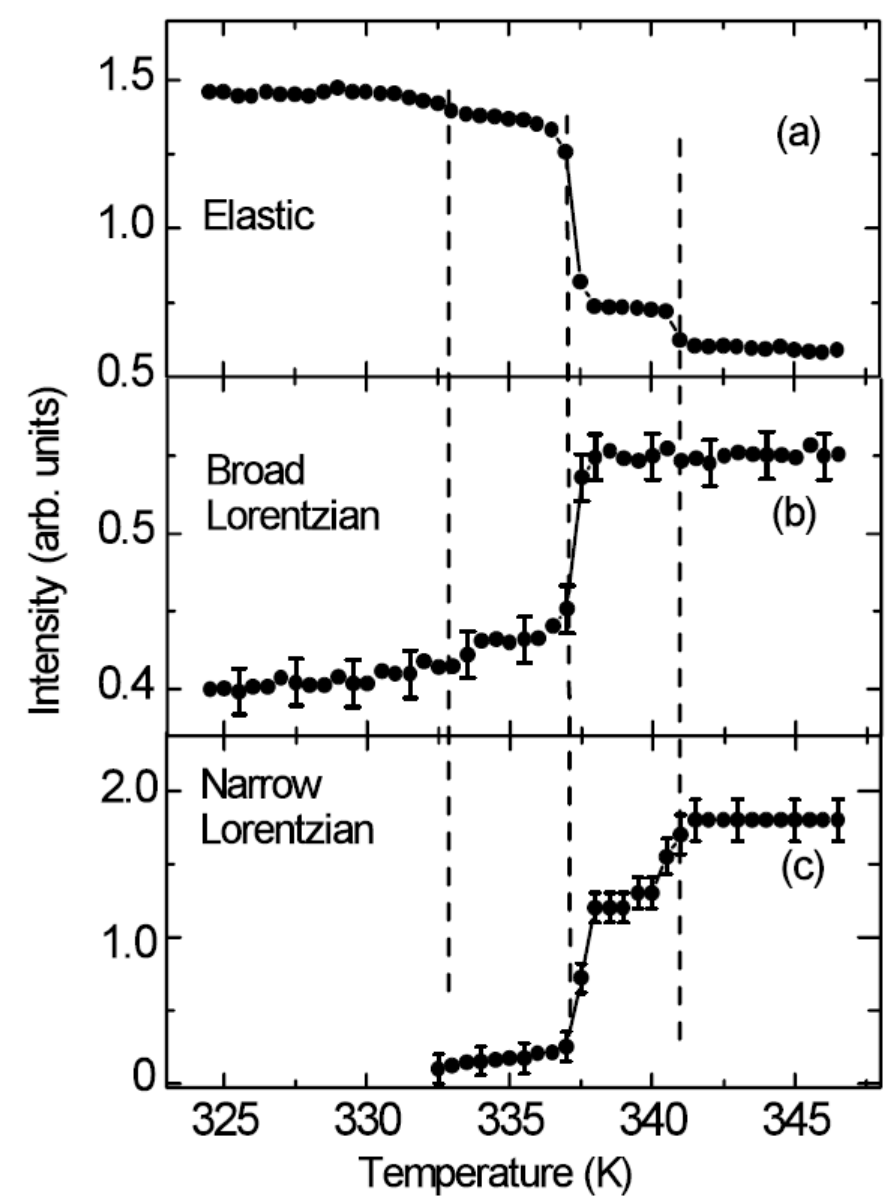

Figure A.1: Intensity of the broad and narrow Lorentzian and elastic components of the quasielastic spectra for $\mathrm{C}_{32}$ particles measured on BASIS. The values in (a) correspond to $\int_{0}^{4 \pi} A(Q, T) d \Omega$; that is, summed over all $Q$ values. The values in (b) and (c) correspond to the intensities of the broad and narrow Lorentzian components summed over all $Q$ values, respectively. Reproduced from Ref. [90]. 
has been reached.

Furthermore, at low temperature $(324.5 \mathrm{~K})$, the elastic intensity also reaches a limiting value. The value of the elastic intensity at low temperature is proportional to scattering from both silicon $\left(\sigma_{S i}\right)$ and from frozen hydrogen nuclei in the alkane molecules $\left(\sigma_{H}\right)$. In other words,

$$
\begin{gathered}
\text { All detectors } A(Q, T=346.5 K) d \Omega=0.55=\alpha \sigma_{S i} \equiv A_{1 H} \\
\qquad \int^{\text {All detectors }} A(Q, T=324.5 K) d \Omega=1.48=\alpha\left(\sigma_{S i}+\sigma_{H}\right) \equiv A_{1 L} .
\end{gathered}
$$

where the subscripts $L$ and $H$ mean "low temperature" and "high temperature", respectively and the subscript "1" means sample 1 (C32). Note that we can sum only over all available $Q$ space, which is limited by the solid angle subtended by the detectors. We then form the expression

$$
\frac{A_{1 L}-A_{1 H}}{A_{1 H}}=\frac{\sigma_{H}}{\sigma_{S i}}=\frac{1.48-0.55}{0.55}=1.93,
$$

Referring to Figure 3.7, the same assumptions apply for the DMPC sample. At high temperature, the elastic intensity is proportional to the cross section of silicon, which we have defined to be unity. At low temperature, the elastic intensity is proportional to the cross section of silicon, DMPC, and water. The cross section of the DMPC membrane is negligible compared to water. Again,

$$
\int^{\text {All detectors }} A(Q, T=315 K) d \Omega=1=\alpha \sigma_{S i} \equiv A_{2 H}
$$




$$
\int A(Q, T=255 K) d \Omega=18.8=\alpha\left(\sigma_{S i}+\sigma_{H_{2} O}\right) \equiv A_{2 L}
$$

Finally,

$$
\frac{A_{1 L}-A_{1 H}}{A_{1 H}} / \frac{A_{2 L}-A_{2 H}}{A_{2 H}}=\frac{\sigma_{H}}{\sigma_{H_{2} O}}=0.113
$$

which expresses the ratio of cross sections of hydrogen in the alkane film to the water in the DMPC system. The subscript "2" here means sample 2 (DMPC).

The total number of hydrogen nuclei in the alkane system is known from AFM measurements; therefore, it is straightforward to calculate the number of water molecules given the above equation. In this case, for the Wet DMPC sample from Figure 3.7, $\sigma_{\mathrm{H}_{2} \mathrm{O}}=2.54 \times 10^{23} \mathrm{~b}$ or $1.53 \times 10^{21} \mathrm{H}_{2} \mathrm{O}$ molecules. For ease of visualization, we assume that the water molecules are at bulk density in a film of thickness $d$ and cover a surface area equal to the surface area of both sides of 100, 2-inch-diameter silicon wafers such that

$$
100 \times 2 \times \pi(2.54 \mathrm{~cm})^{2} \times d=\frac{m_{H_{2} O}}{\rho_{H_{2} O}} \times \frac{N}{N_{A}} .
$$

where $m_{H 2 O}$ is the mass of a water molecule, $\rho_{H 2 O}$ is the bulk density of water, and $N_{A}$ is Avogadro's number.

We have equated the volume of such a film to the volume of the calculated number of water molecules. For the protonated water DMPC sample described here, the film thickness $d$ is $113 \mathrm{~nm}$. We repeat the calculation for the other DMPC samples. In the following tables, the labels p-72 and d-54+p-18 represent fully protonated (72 hydrogens) DMPC and tail-deuterated (54 deuterium and 18 hydrogen nuclei) DMPC, respectively. 
Table A.1: Water content for lipid samples

\begin{tabular}{|l|l|l|}
\hline Sample Name & Lipid Deuteration & Water Deuteration \\
\hline Wet 1 & p-72 & $\mathrm{H}_{2} \mathrm{O}$ \\
\hline Wet 2 & $\mathrm{p}-72$ & $\mathrm{H}_{2} \mathrm{O}$ \\
\hline Dry & d-54+p-18 & $\mathrm{H}_{2} \mathrm{O}$ \\
\hline Superdry & p-72 & $\mathrm{H}_{2} \mathrm{O}$ \\
\hline Wet-D & p-72 & $\mathrm{D}_{2} \mathrm{O}$ \\
\hline
\end{tabular}

\begin{tabular}{|l|c|c|c|}
\hline Sample Name & Water Volume $(\mu \mathrm{l})$ & Water molecules per Lipid & Slab Thickness $(\AA)$ \\
\hline Wet 1 & 58.1 & 1,560 & 1,130 \\
\hline Wet 2 & 42.7 & 1,140 & 829 \\
\hline Dry & 5.73 & 154 & 111 \\
\hline Superdry & - & - & - \\
\hline Wet-D & - & - & - \\
\hline
\end{tabular}

Table A.2: Water content of the DMPG samples

\begin{tabular}{|l|c|c|c|}
\hline Sample Name & Water Volume $(\mu \mathrm{l})$ & Water molecules per Lipid & Film Thickness $(\AA)$ \\
\hline Dry 1 & 12.0 & 318 & 233 \\
\hline Dry 2 & 19.8 & 528 & 386 \\
\hline Wet 1 & 26.7 & 711 & 520 \\
\hline Wet 2 & 36.5 & 972 & 711 \\
\hline
\end{tabular}

The calculation is identical for DMPG samples. The results are tabulated below. 


\section{Appendix B}

\section{List of Samples Investigated on HFBS and BASIS}

This Appendix contains a comprehensive list of samples measured on the HFBS and BASIS from August 2009 to September 2013. Sample fabrication parameters, dates of measurement, protonation, and figures in which these samples appear are provided. Selected fixed window scans of samples of particular importance which do not otherwise appear in the previous chapters are presented here as well. Generally, fixed window scans are collected on more than one thermal cycle for each sample; however, the most representative thermal cycle is selected and shown in the figures below.

Sample \#1 - Protonated DMPC annealed for 3 days at $55^{\circ} \mathrm{C}$. $120 \mu \mathrm{l}$ of $\mathrm{D}_{2} \mathrm{O}$ was added to the sample can prior to sealing. Measured on HFBS in August 2009.

Sample \#2 - d54-DMPC annealed for 3 days at $55{ }^{\circ} \mathrm{C} .120 \mu$ of $\mathrm{H}_{2} \mathrm{O}$ was added to the sample can prior to sealing. Measured on HFBS in August 2009.

Sample \#3 - Protonated DMPC annealed for 3 days at $55^{\circ} \mathrm{C}$. $120 \mu \mathrm{l}$ of $\mathrm{H}_{2} \mathrm{O}$ was 
added to the sample can prior to sealing. Measured on HFBS in April 2010. Appears in Fig. 3.2 as Wet 2. Appears in Fig. 3.3.

Sample \#4 - d54-DMPC annealed for 3 days at $55{ }^{\circ} \mathrm{C}$. Produced in $\mathrm{H}_{2} \mathrm{O}$ buffer solution but no water added to can prior to sealing. Measured on HFBS in November 2010. Measured on BASIS in October, 2010. Appears in Fig. 3.13 as Superdry.

Sample \#5 - Protonated DMPC annealed for 3 days at $55^{\circ} \mathrm{C} .120 \mu$ l of $\mathrm{H}_{2} \mathrm{O}$ was added to the sample can prior to sealing. Measured on HFBS in November, 2010. Measured on BASIS in May 2010. Appears in Fig. 3.2 as Wet 1. Appears in Fig. 3.7 as Wet 1. Appears in Fig. 3.4.

Sample \#6 - Protonated DMPC annealed for 3 days at $55^{\circ} \mathrm{C} .120 \mu \mathrm{l}$ of $\mathrm{D}_{2} \mathrm{O}$ was added to the sample can prior to sealing. Measured on HFBS in November, 2010. Measured on BASIS in October 2010. Appears in Fig. 3.11 as Wet-D (Membrane).

Sample \#7 - d54-DMPC produced in $\mathrm{H}_{2} \mathrm{O}$ buffer solution. Sample was not annealed. Measured on HFBS in November 2010. Measured on BASIS in October, 2010. Appears in Fig. 3.2 as Dry. Appears in Fig. 3.7 as Dry.

Sample \#8 - Bare silicon sample. $120 \mu \mathrm{l}$ of $\mathrm{H}_{2} \mathrm{O}$ was added to the can prior to sealing. Measured on HFBS in November 2012.

Sample \#9 - Protonated DMPG produced in $\mathrm{H}_{2} \mathrm{O}$ buffer solution but no water added to can prior to sealing. Measured on HFBS in November,2012.

Sample \#10 - Protonated DMPG annealed for 3 days at $55^{\circ} \mathrm{C} .120 \mu$ l of $\mathrm{H}_{2} \mathrm{O}$ was added to the sample can prior to sealing. Measured on HFBS in November 2012.

Sample \#11 - Protonated DMPG annealed for 3 days at $55{ }^{\circ} \mathrm{C} .120 \mu \mathrm{l}$ of $\mathrm{H}_{2} \mathrm{O}$ was added to the sample can prior to sealing. Measured on HFBS in November 2012. Measured on HFBS in April 2013. Measured on BASIS in September 2013. Appears 
in Fig. 4.1 as Wet 2.

Sample \#12 - Protonated DMPG annealed for 3 days at $55 \circ \mathrm{C} .100 \mu \mathrm{l}$ of $\mathrm{H}_{2} \mathrm{O}$ was added to the sample can prior to sealing. Measured on HFBS in April 2013. Appears in Fig. 4.1 as Wet 1.

Sample \#13 - Protonated DMPG produced in $\mathrm{H}_{2} \mathrm{O}$ buffer solution but no water added to can prior to sealing. Measured on HFBS in April 2013. Measured on BASIS in September 2013. Appears in Fig. 4.1 as Dry 1.

Sample \#14 - Protonated DMPG produced in $\mathrm{H}_{2} \mathrm{O}$ buffer solution but no water added to can prior to sealing. Measured on HFBS in April 2013. Appears in Fig. 4.1 as Dry 2. 
Table B.1: List of samples measured on HFBS

\begin{tabular}{|c|c|c|c|}
\hline Sample Name & Date of Fabrication & Date of Run & Figures \\
\hline$\# 1$ (DMPC) & Aug. 09 & $9 / 8 / 09$ & - \\
\hline$\# 2$ (DMPC) & Aug. 09 & $9 / 8 / 09$ & - \\
\hline \#3 (DMPC) & Mar. 10 & $4 / 15 / 10$ & $3.2,3.3$ \\
\hline \#4 (DMPC) & Nov. 10 & $11 / 8 / 10$ & 3.13 \\
\hline \#5 (DMPC) & Nov. 10 & $11 / 15 / 10$ & $3.2,3.7,3.4$ \\
\hline \#6 (DMPC) & Nov. 10 & $11 / 09 / 10$ & 3.11 \\
\hline \#7 (DMPC) & Nov. 10 & $11 / 10 / 10$ & $3.2,3.7$ \\
\hline$\# 8(\mathrm{Si})$ & Nov. 10 & $11 / 10 / 10$ & - \\
\hline \#9 (DMPG) & Oct. 12 & $11 / 6 / 12$ & - \\
\hline \#10 (DMPG) & Oct. 12 & $11 / 9 / 12$ & - \\
\hline \#11 (DMPG) & Oct. 12 & $11 / 5 / 12,4 / 22 / 13$ & 4.1 \\
\hline \#12 (DMPG) & Apr. 13 & $4 / 26 / 13$ & 4.1 \\
\hline$\# 13$ (DMPG) & Apr. 13 & $4 / 24 / 13$ & 4.1 \\
\hline$\# 14$ (DMPG) & Apr. 13 & $4 / 28 / 13$ & 4.1 \\
\hline
\end{tabular}

Table B.2: List of samples measured on BASIS

\begin{tabular}{|l|c|c|c|}
\hline Sample Name & Date of Fabrication & Date of Run & Figures \\
\hline \#4 (DMPC) & Sep. 10 & $10 / 13 / 10$ & 3.13 \\
\hline \#5 (DMPC) & May 10 & $11 / 15 / 10$ & $3.2,3.7,3.4$ \\
\hline$\# 6$ (DMPC) & Oct. 10 & $10 / 15 / 10$ & 3.11 \\
\hline \#7 (DMPC) & Oct. 10 & $10 / 17 / 10$ & $3.2,3.7$ \\
\hline$\# 11$ (DMPG) & Oct. 12 & $9 / 9 / 13$ & 4.1 \\
\hline$\# 13$ (DMPG) & Apr. 12 & $9 / 13 / 13$ & 4.1 \\
\hline
\end{tabular}




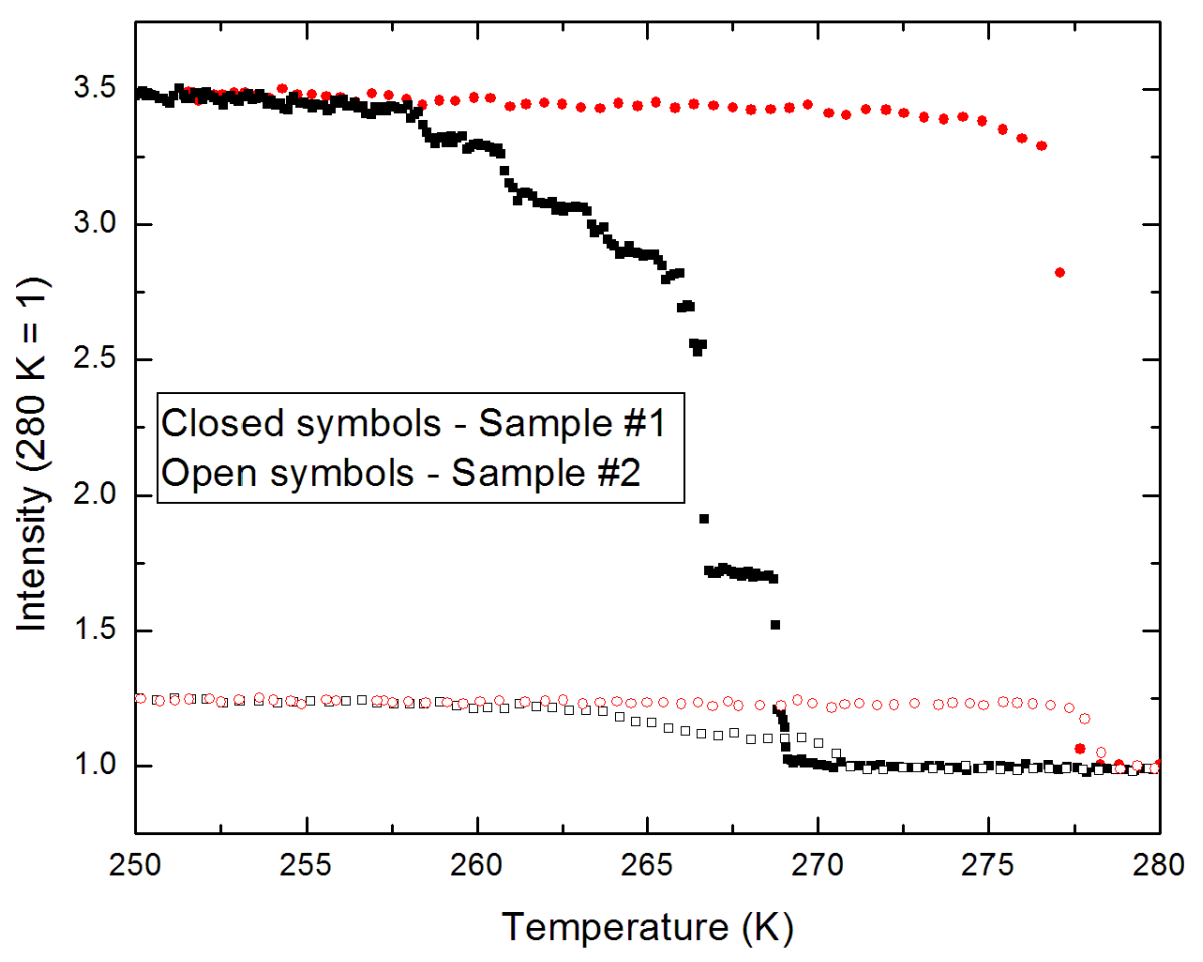

Figure B.1: Fixed window scans of DMPC samples fabricated in August, 2009. Closed symbols represent Sample \#1 produced with $\mathrm{H}_{2} \mathrm{O}$ and d54-DMPC. Open symbols represent Sample \#2 produced with $\mathrm{D}_{2} \mathrm{O}$. Black symbols are measured on cooling. Red symbols are measured on heating. The $\mathrm{D}_{2} \mathrm{O}$ sample shows much lower intensity at low temperature due to the incoherent cross section of $\mathrm{D}$ being 40 times smaller than H. Because the temperature ramp for Sample $\# 1$ was $0.3 \mathrm{~K} / \mathrm{min}$ on heating, the intensity decreases in a step at $277 \mathrm{~K}$, several degrees above the bulk melting point. Intensity is normalized such that the intensity at $280 \mathrm{~K}$ is unity. These samples were the earliest to be measured on the HFBS. 


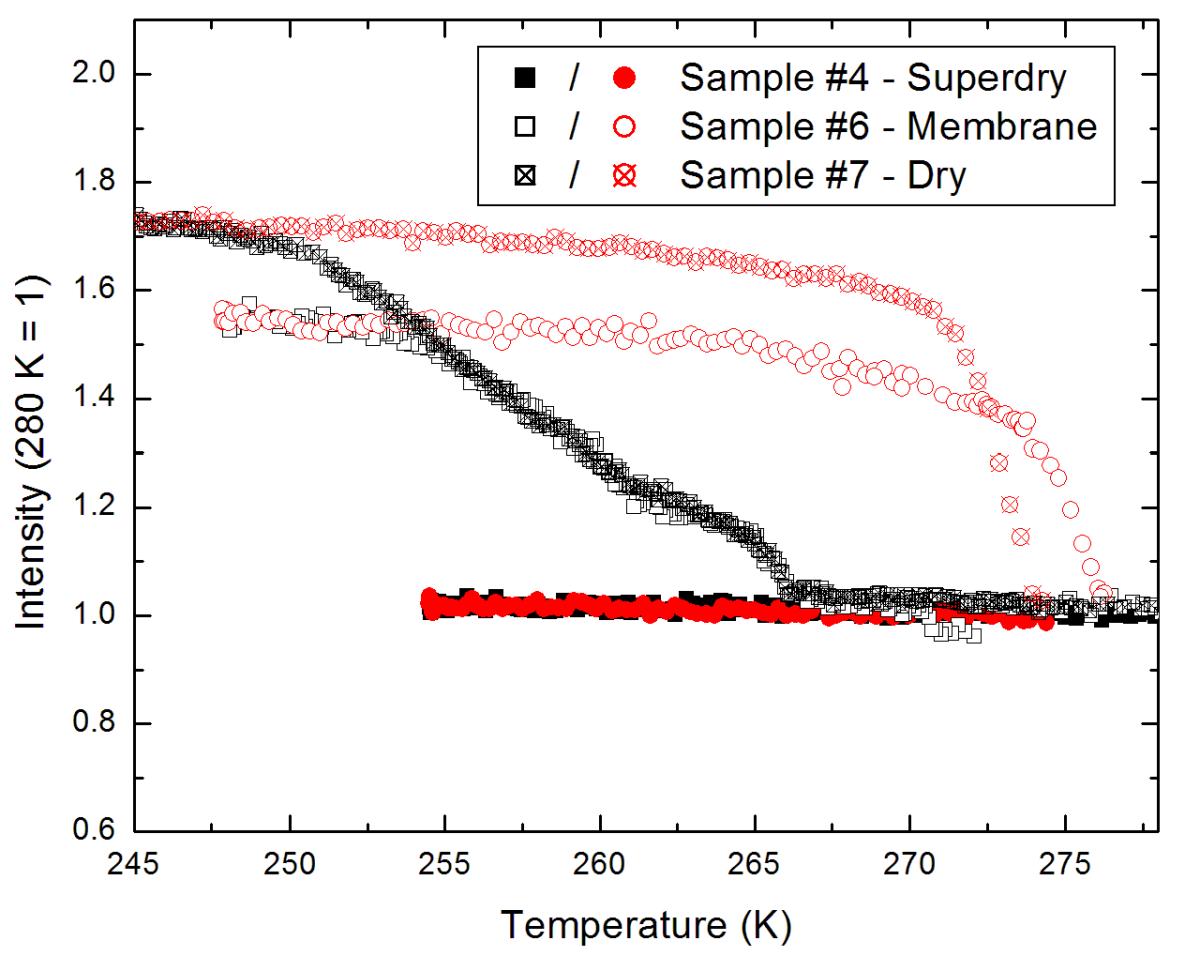

Figure B.2: Fixed window scans of DMPC samples. Black points are taken on cooling, red points are taken on heating. The closed symbols (Sample \#4, Superdry) represent the least amount of water because the sample was produced with d54-DMPC and annealed for $72 \mathrm{~h}$ without rehydration. Open symbols represents the "Membrane" sample with $\mathrm{D}_{2} \mathrm{O}$ but protonated lipids. This sample contained as much water as the Wet 1 sample in Figure 3.3, but the intensity is much lower due to the cross section of D. Furthermore, the melting point of $\mathrm{D}_{2} \mathrm{O}$ is $277 \mathrm{~K}$ compared to $273 \mathrm{~K}$ for $\mathrm{H}_{2} \mathrm{O}$, which explains the difference between the Membrane sample and the crossed symbols (Sample \#7, Dry, a sample produced with a small amount of $\mathrm{H}_{2} \mathrm{O}$ ). 


\section{Appendix $\mathrm{C}$}

\section{Theory of Neutron Scattering Cross Sections}

Any neutron scattering experiment is concerned with determining the behavior of the energy transfer, $\hbar \omega$, and momentum transfer, $\mathbf{Q}$, of the neutron as a function of some externally varied parameter, such as temperature or magnetic field. The details of the interaction of the neutron with the sample are described by the double-differential cross section

$$
\frac{1}{\hbar} \frac{\partial^{2} \sigma}{\partial \boldsymbol{\Omega} \partial \omega}
$$

with $\frac{\partial \sigma}{\partial \boldsymbol{\Omega}}$ representing the probability of a neutron scattering in the direction of the solid angle element $\Omega$ and $\frac{\partial \sigma}{\partial \omega}$ representing the probability of a neutron transferring an energy $\Delta E=\hbar \omega=E_{i}-E_{f}$ to the sample. The double-differential cross section 
is defined to have units of area/energy. This quantity is what is actually measured in a neutron scattering experiment.

Neutrons interact with matter in three ways. The neutron interacts with nuclear spin via a dipole-dipole interaction which is very weak and ignored here. Furthermore, the neutron has a magnetic moment that interacts with unpaired electrons to give rise to magnetic scattering. Because this interaction occurs between the outermost electrons, which are spread over the volume of the entire atom, magnetic scattering is not isotropic and has a strong $Q$ dependence. Generally speaking, neutrons interact magnetically with atoms that are themselves highly magnetic. For the work presented here, the primary atomic constituents of samples are light elements such as hydrogen, oxygen, and carbon, which are not magnetic. Thus, magnetic scattering will not be covered here.

The most dominant form of neutron scattering for most materials is via the nuclear interaction. Typical neutron wavelengths are on the order of 1-6 $\AA$, compared with the diameter of the nucleus on the order of $10^{-14} \mathrm{~m}$. Because of this difference in length scale the neutron is insensitive to local variations in the nuclear scattering potential; therefore, nuclear scattering from single nuclei is isotropic. Furthermore, the nuclear scattering potential, because it is short range, can be written in the following way

$$
V(\mathbf{r})=\frac{2 \pi \hbar^{2}}{m} b \delta(\mathbf{r}-\mathbf{R})
$$


where $m$ is the mass of the neutron which interacts with a nucleus at position $\mathbf{R}[41]$. The above expression is called the Fermi pseudopotential. The quantity $b$ is called the scattering length of the nucleus. Because nuclear scattering is isotropic, it is only necessary to describe the interaction with a single parameter; no angular parameter is necessary to fully describe the potential.

The neutron interacts with the spin states of the nucleus. Thus, the scattering length of an isolated nucleus can take a range of values among the available nuclear spin states which are sampled randomly. Neutrons interact with a large number of nuclei simultaneously; furthermore, unless exchange forces are relevant, spin states of neighboring nuclei are uncorrelated. Therefore, a neutron scattering experiment is a probe of the average scattering length of the system as well as the fluctuation from the mean value. For a given isotope, it is common to differentiate between coherent and incoherent scattering such that

$$
\begin{gathered}
b_{c o h}=\langle b\rangle \\
b_{i n c}=\left[\left\langle b^{2}\right\rangle-\langle b\rangle^{2}\right]^{1 / 2}
\end{gathered}
$$

wherein the averaging is performed over all spin states of that isotope. Coherent scattering is so called because it is responsible for interference effects. When the scattering is averaged over many scattering centers, only the average scattering length 
can give rise to interference effects. On the other hand, the local deviation of the scattering length from the average value gives rise to incoherent scattering, because the result is not correlated with its neighbors by definition. In practice, the incoherent scattering length is the relevant factor for any scattering process which occurs at a single nucleus. The coherent scattering length describes the strength of scattering which is the result of interference from more than one nucleus.

The neutron is a spin $\frac{1}{2}$ particle; therefore, if we consider a nucleus with spin $S$ then the two available spin states of the nucleus after scattering are $S_{+}=S+1 / 2$ and $S_{-}=S-1 / 2$. Associated with $S_{+}$and $S_{-}$are the scattering lengths $b_{+}$and $b_{-}$. If $S_{+}$and $S_{-}$are equally probable then

$$
\begin{gathered}
\langle b\rangle=\frac{1}{2 S_{+}+2 S_{-}+2}\left[\left(2 S_{+}+1\right) b_{+}+\left(2 S_{-}+1\right) b_{-}\right]=b_{c o h} \\
\left\langle b^{2}\right\rangle=\frac{1}{2 S_{+}+2 S_{-}+2}\left[\left(2 S_{+}+1\right) b_{+}^{2}+\left(2 S_{-}+1\right) b_{-}^{2}\right] \\
b_{i n c}=\frac{1}{S_{+}+S_{-}+1}\left[1 / 2\left(S_{+}+S_{-}+2\right) b_{+}+1 / 2\left(S_{+}+S_{-}\right) b_{-}\right] .
\end{gathered}
$$

The determination of $b_{+}$and $b_{-}$depends on the details of the nuclear interaction. For elements heavier than deuterium or helium, the theoretical calculation of $b_{+}$and $b_{-}$is difficult or impossible. For hydrogen, however, the relevant scattering lengths are known: 


$$
\begin{gathered}
b_{+}=1.04 \times 10^{-14} \mathrm{~cm} \\
b_{-}=-4.74 \times 10^{-14} \mathrm{~cm} . \\
\langle b\rangle=\frac{1}{4}\left[3 b_{+}+b_{-}\right]=-0.38 \times 10^{-12} \mathrm{~cm} \\
\left\langle b^{2}\right\rangle=\frac{1}{4}\left[3 b_{+}^{2}+b_{-}^{2}\right]=6.49 \times 10^{-24} \mathrm{~cm}^{2}
\end{gathered}
$$

such that

$$
\begin{gathered}
\sigma_{c o h}(H)=1.8 \mathrm{~b} \\
\sigma_{\text {inc }}(H)=79.9 \mathrm{~b}
\end{gathered}
$$

where $1 \mathrm{~b}=10^{-24} \mathrm{~cm}^{2}$ and $\sigma_{c o h}=4 \pi\langle b\rangle^{2}$ and $\sigma_{i n c}=4 \pi\left[\left\langle b^{2}\right\rangle-\langle b\rangle^{2}\right]$. Hydrogen is a special case because the total cross section of hydrogen is larger than any other nucleus (except a few heavy rare earth isotopes). The explanation for hydrogen's exceptionally large total cross section is the coincidental fact that the scattering lengths for its singlet and triplet state are opposite sign but differ almost by their multiplicity, maximizing the above expression.

Because hydrogen is so abundant in organic compounds it is a clear target for neutron scattering. Furthermore, its chemical partner deuterium has the following scattering cross sections: 


$$
\begin{gathered}
\sigma_{c o h}(D)=5.6 \mathrm{~b} \\
\sigma_{\text {inc }}(D)=2.0 \mathrm{~b} .
\end{gathered}
$$

Therefore, it is a common approach to replace $\mathrm{H}$ with $\mathrm{D}$ in order to selectively probe coherent or incoherent scattering from a sample. Doing so does not affect the chemistry of the sample. Finally, because the neutron interaction is determined by the interaction with the spin states (described by $b_{+}$and $b_{-}$), which are not monotonic functions of the number of protons and neutrons in the nucleus, the neutron is not limited to focusing exclusively on heavy isotopes which are favorable for x-ray scattering. Several of the most common elements in organic materials including C, $\mathrm{O}$, and $\mathrm{N}$ all have relatively comparable cross sections to heavier elements which, in addition to hydrogen, makes neutron scattering an ideal tool for investigating organic materials. 


\section{Appendix D}

\section{Determining the Uncertainty in Diffusion Constants}

In Chapter 1, we derived an expression for the dynamic structure factor for water diffusion:

$$
S_{i n c}(Q, \omega)=\frac{1}{\pi} \frac{D Q^{2}}{\omega^{2}+\left(D Q^{2}\right)^{2}} .
$$

The HWHM of this Lorentzian function is $\Gamma=D Q^{2}$. By fitting the HWHM as a function of $Q$, the diffusion constant $D$ for a particular temperature can be determined by applying a weighted least-squares fit to $\Gamma$ vs. $Q^{2}$. The fit is performed by minimizing

$$
\chi^{2}=\sum\left[\frac{1}{\sigma_{i}^{2}}\left(\Gamma_{i}-a-b Q_{i}^{2}\right)^{2}\right]
$$

where $\Gamma_{i}$ is the HWHM at a particular $Q_{i}^{2}$. a will be the intercept of $\Gamma$ which we demand to be zero. $b$ will be the diffusion constant. The $\sigma_{i}$ are the standard deviation 
of each $\Gamma$ and are unique for each point. Because $\Gamma$ is itself a fitted quantity, it must be determined separately. We will discuss the derivation of $\sigma_{i}$ later. We can set $a=0$ now:

$$
\chi^{2}=\sum\left[\frac{1}{\sigma_{i}^{2}}\left(\Gamma_{i}-b Q_{i}^{2}\right)^{2}\right]
$$

Next we minimize $\chi^{2}$ by setting its derivative to zero.

$$
\frac{d}{d b} \chi^{2}=-2 \sum\left[\frac{Q_{i}^{2}}{\sigma_{i}^{2}}\left(\Gamma_{i}-b Q_{i}^{2}\right)\right]=0
$$

Since we only have $b$ we can rearrange and solve this analytically.

$$
\begin{gathered}
0=\left[\sum \frac{Q_{i}^{2} \Gamma_{i}}{\sigma_{i}^{2}}-b \frac{\left(Q_{i}^{2}\right)^{2}}{\sigma_{i}^{2}}\right] \\
b=\frac{\sum \frac{Q_{i}^{2} \Gamma_{i}}{\sigma_{i}^{2}}}{\sum \frac{\left(Q_{i}^{2}\right)^{2}}{\sigma_{i}^{2}}}
\end{gathered}
$$

Therefore $b$ is determined. To determine the uncertainty in $b$ we follow the propagation of errors method. For any quantity,

$$
\sigma_{z}^{2}=\sum\left[\sigma_{i}^{2}\left(\frac{d z}{d c_{i}}\right)^{2}\right]
$$

where $\sigma_{i}$ is the standard deviation in the point $c_{i}$. Here we want to find the uncertainty in $b$ so we take the derivative of Eq. D.6.

$$
\frac{d b}{d \Gamma_{i}}=\frac{\frac{Q_{i}^{2}}{\sigma_{i}^{2}}}{\sum \frac{\left(Q_{i}^{2}\right)^{2}}{\sigma_{i}^{2}}}
$$

and insert them into Eq. D.7 


$$
\begin{gathered}
\sigma_{b}^{2}=\sum \sigma_{i}^{2}\left(\frac{d b}{d \Gamma_{i}}\right)^{2} \\
\sigma_{b}^{2}=\sum \sigma_{i}^{2}\left(\frac{\frac{Q_{i}^{2}}{\sigma_{i}^{2}}}{\sum \frac{\left(Q_{j}^{2}\right)^{2}}{\sigma_{j}^{2}}}\right)^{2} .
\end{gathered}
$$

The $b$ and $\sigma_{b}$ values are the diffusion constant and their uncertainties as they appear in Fig. 3.10.

The $Q^{2}$ values are fixed in the experiment. The $\Gamma_{i}$ values are determined by applying a least-squares fit for the scattering law (Eq. 3.1) to the experimental data. However, it was found that the uncertainties in $\Gamma$, which would normally be determined in a similar way as described here, were not reasonable due to systematic errors in the fitting procedure. In particular, there was a significant tendency for the least-squares fit to prefer unphysical local minima in the fitting parameters. For instance, the quality of the fit could be similar between a fit with a large linear background + low-intensity Lorentzian term and a small linear background + highintensity Lorentzian term. This effect is illustrated in Fig. D.1. In practice, it is necessary to invoke an additional physical constraint to choose the correct fit. The procedure described here for determining the uncertainty in a least-squares fit does not consider this systematic uncertainty.

In order to determine the uncertainty in $\Gamma$, we note that $\chi^{2}$ is proportional to $\Gamma^{2}$ at low $Q$ because $\chi^{2}$ here is the square difference between a Lorentzian function (linear in $\Gamma$ )and the data. We propose that the deviation of a parabolic fit to $\chi^{2}$ vs. $\Gamma$ will give an indication of the size of the systematic uncertainty associated with the presence of additional local minima. The parabolic fit is shown in Fig. D.2. 


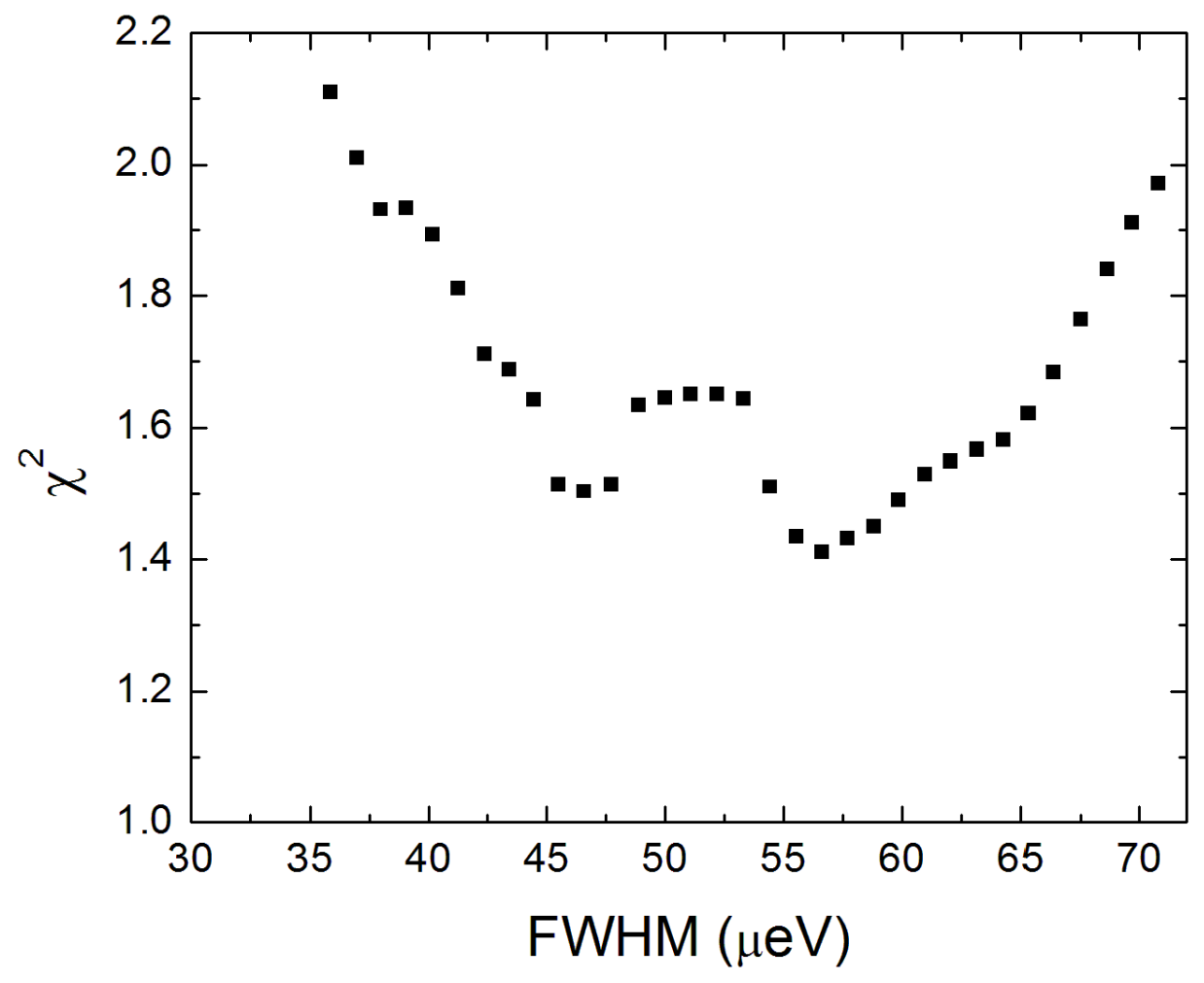

Figure D.1: $\chi^{2}$ of fit as a function of FWHM of broad component for $Q=0.7 \AA$ at $270 \mathrm{~K}$ for the Wet DMPC sample. The best-fit value is $56.6 \mu \mathrm{eV}$; however, a second local minima appears at $46.5 \mu \mathrm{eV}$ corresponding to a solution with a large linear background. The intensity (not shown) of the broad Lorentzian for the $46.5 \mu \mathrm{eV}$ FWHM fit is significantly lower than the $56.6 \mu \mathrm{eV}$ value. 


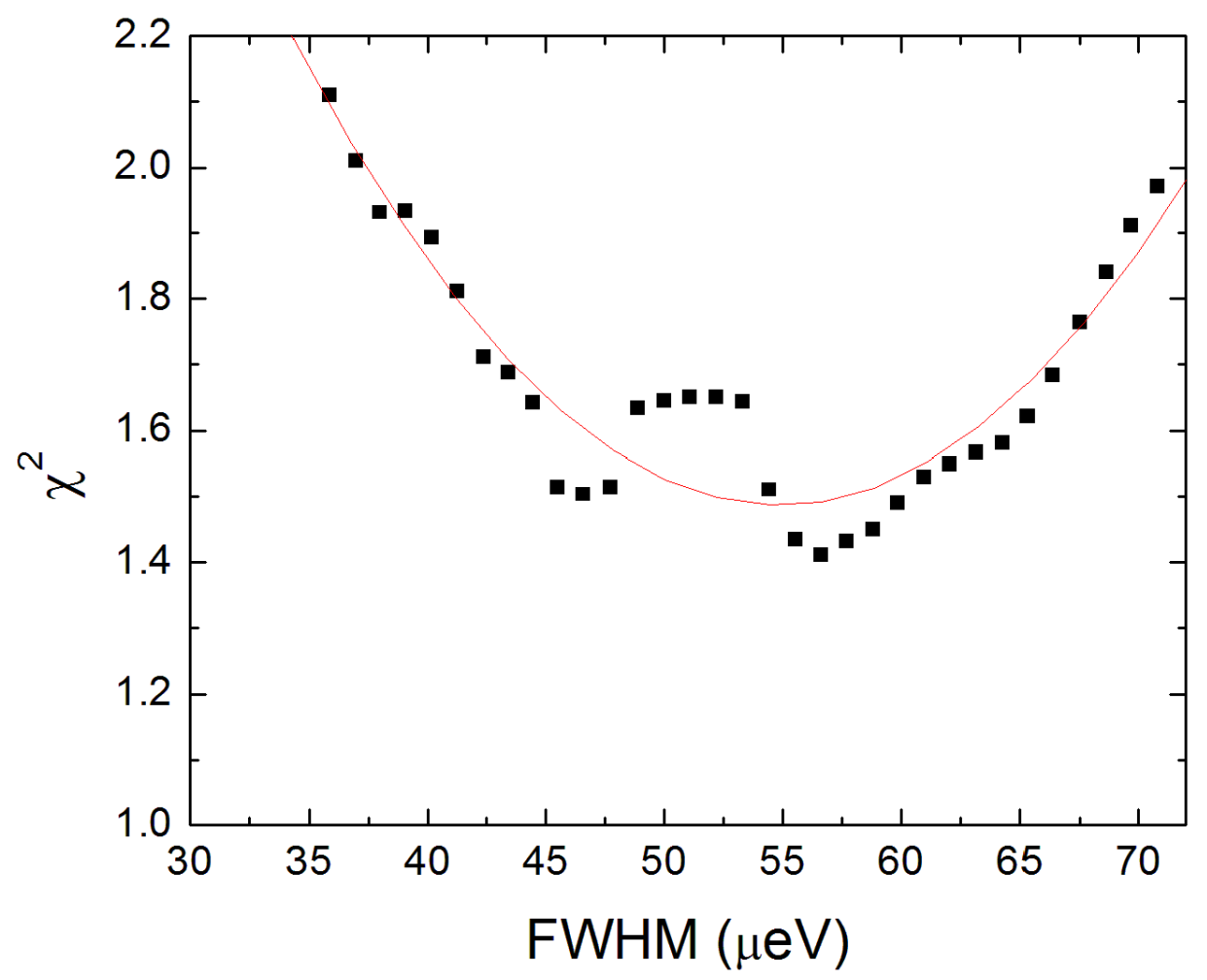

Figure D.2: Parabolic fit of $\chi^{2}$ as a function of FWHM of broad component for $Q=0.7 \AA$ at $270 \mathrm{~K}$ for the Wet DMPC sample. The standard deviation of the fit is an indication of the effect of the local minimia at $46.5 \mu \mathrm{eV}$ because the $\chi^{2}$ value is expected to be proportional to $\Gamma^{2}$.

The standard deviation calculated in this way is $3.9 \mu \mathrm{eV}$, significantly higher than the error calculated without this systematic uncertainty $(0.7 \mu \mathrm{eV})$. The uncertainty calculated in this way are the error bars in Figs. 3.8 and 3.9 and they are the values $\sigma_{i}$ appearing in Eq. D.3. 


\section{Bibliography}

[1] F. Y. Hansen, G. H. Peters, H. Taub, and A. Miskowiec, J. Chem. Phys. 137, $204910(2012)$.

[2] K. Blodgett and I. Langmuir, Phys. Rev. 51, 964 (1937).

[3] K. Blodgett, J. Am. Chem. Soc. 57, 1007 (1935).

[4] I. Langmuir, J. Chem. Soc. Faraday Trans. 15, 62 (1920).

[5] L. Tamm and H. M. McConnel, Biophys. J. 47, 105 (1985).

[6] E. T. Castellana and P. S. Cremer, Surf. Sci. Rep. 61, 429 (2006).

[7] N. Bunjes, E. K. Schmidt, A. Jonczyk, F. Rippman, D. Beyer, H. Ringsdorf, P. Graeber, W. Knoll, and R. Naumann, Langmuir 13, 6188 (1997).

[8] P. S. Cremer and S. G. Boxer, J. Phys. Chem. B 103, 2554 (1999).

[9] S. Gritsch, P. Nollert, F. Jahnig, and E. Sackmann, Langmuir 14, 3118 (1998).

[10] B. C. Lagerholm, T. E. Starr, Z. N. Volovyk, and N. L. Thompson, Biochemistry 39, 2042 (2000). 
[11] J. Lahiri, P. Kalal, A. G. Frutos, S. J. Jonas, and R. Schaeffler, Langmuir 16, $7805(2000)$.

[12] G. Puu and I. Gustafson, Biochim. Biophys. Acta 1327, 149 (1997).

[13] T. E. Starr and N. L. Thompson, Langmuir 16, 10301 (2000).

[14] J. Yang and M. Kleijn, Biophys. J. 76, 323 (1999).

[15] J. A. Zasadzinski, C. A. Helm, Marjorie L. Longo, A. Weisenhorn, S. A. C. Gould, and P. K. Hasnma, Biophys. J. 59, 756 (1991).

[16] T. Baumgart and A. Offenhusser, Langmuir 19, 1730 (2003).

[17] J. Spinke, J. Yang, H. Wolf, M. Liley, H. Ringsorf, and W. Knoll, Biophys. J. 63, 1667 (1992).

[18] T. Wang, D. Li, X. Lu, A. Khmaladze, X. Han, S. Ye, P. Yang, G. Xue, N. He, and Z. Chen, J. Phys. Chem. C 115, 7613 (2011).

[19] M. Edidin, Annu. Rev. Bioph. Biom. 32, 257 (2003).

[20] A. G. Lee, Biochim. Biophys. Acta 1666, 62 (2004).

[21] R. Naumann, A. Jonczyk, R. Kopp, J. van Esch, H. Ringsdorf, W. Knoll, and P. Graeber, Angew. Chem. Int. Edit. 34, 2056 (1995).

[22] L. Renner, T. Osaki, S. Chiantia, P. Schwille, T. Pompe, and C. Werner, J. Phys. Chem. B 112, 6373 (2008).

[23] J. Mou, J. Yang, C. Huang, and Z. Shao, Biochemistry 33, 9981 (1994). 
[24] Y. Fang and J. Yang, Biochim. Biophys. Acta 1324, 309 (1997).

[25] T. Schmidt, G. J. Schutz, W. Baumgartner, H. J. Gruber, and H. Schindler, P. Natl. Acad. Sci. USA 93, 2926 (1996).

[26] P. F. F. Almeida, W. L. Vaz, and T. E. Thimpson, Biochemistry 31, 6739 (1992).

[27] Z. Derzko and K. Jacobson, Biochemistry 19, 6050 (1980).

[28] J. F. Nagle and S. Tristram-Nagle, Curr. Opin. Struc. Biol. 10, 474 (2000).

[29] K. A. Riske, H-G. Dobereiner, and M. T. Lamy-Freund, J. Phys. Chem. B 106, 239 (2002).

[30] Z. V. Feng, T. A. Spurlin, and A. A. Gewirth, Biophys. J. 88, 2154 (2005).

[31] S. Garcia-Manyes, G. Oncins, and F. Sanz, Biophys. J. 89, 4261 (2005).

[32] A. Xie, R. Yamada, A. Gewirth, and S. Granick, Phys. Rev. 89, 246100 (2002).

[33] O. Enders, A. Ngezahayo, M. Wiechmann, F. Leisten, and H. A. Kolb, Biophys. J. 87, 2522 (2004).

[34] G. Cevc, A. Watts, and D. Marsh, Biochemistry 20, 4955 (1981).

[35] H. A. Rinia, R. A. Demel, J. P. J. M. van der Eerden, and B. De Kruijff, Biophys. J. 77, 1683 (1999).

[36] W. Zhao, T. Rog, A. A. Gurtovenko, I. Vattulainen, and M. Karttunen, Biophys. J. 92, 1114 (2007). 
[37] J. Pimthon, R. Willumeit, A. Lendlein, and D. Hofmann, J. Mol. Struct. 921, $38(2009)$.

[38] Y. Kaznessis, S. Kim, and R. Larson, Biophys. J. 82, 1731 (2002).

[39] D. Grigoriev, R. Krustev, R. Miller, and U. Pison, J. Phys. Chem. B 103, 1013 (1999).

[40] K. A. Riske, O. R. Nascimento, M. Peric, B. Bales, and M. T. Lamy-Freund, Biochim. Biophys. Acta 1418, 133 (1999).

[41] M Bee, Quasielastic neutron scattering, IOP Publishing Ltd, Bristol, England, 1988.

[42] M. Bai, A. Miskowiec, F. Y. Hansen, H. Taub, T. Jenkins, M. Tyagi, S. O. Diallo, E. Mamontov, K. W. Herwig, and S. K. Wang, Europhys. Lett. 98, 48006 (2012).

[43] A. Meyer, R. M. Dimeo, P. M. Gehring, and D. A. Neumann, Rev. Sci. Instrum. 74, 2759 (2003).

[44] E. Mamontov and K. W. Herwig, Rev. Sci. Instrum. 82, 085109 (2011).

[45] J. Swenson, F. Kargl, P. Berntsen, and C. Svanberg, J. Chem. Phys. 129, 045101 (2008).

[46] S. Koenig, E. Sackmann, D. Richter, R. Zorn, C. Carlile, and T. M. Bayerl, J. Chem. Phys. 100, 3307 (1993).

[47] J. Teixeira, M. C. Bellissent-Funel, and A. J. Dianoux, Phys. Rev. A 31, 1913 (1985). 
[48] P. Gallo, F. Sciortino, P. Tartaglia, and S. H. Chen, Phys. Rev. Lett. 76, 2730 (1996).

[49] P. L Hall and Ross D. K., Mol. Phys. 42, 673 (1981).

[50] A. Faraone, L. Liu, C. Y. Mou, C. W. Yen, and S. H. Chen, J. Chem. Phys. 121, 10843 (2004).

[51] S. Mitra, R. Mukhopadhyay, I. Tsukushi, and S. Ikeda, J. Phys.-Condens. Mat. 13, 8455 (2001).

[52] J.-M. Zanotti, M. C. Bellissent-Funel, and S. H. Chen, Phys. Rev. E 59, 3084 (1999).

[53] J. Qvist, H. Schober, and B. Halle, J. Chem. Phys. 134, 144508 (2011).

[54] V. Crupi, D. Majolino, P. Migliardo, and V. Venuti, J. Phys. Chem. B 106, 10884 (2002).

[55] M. C. Bellissent-Funel, Eur. Phys. J. E 12, 83 (2003).

[56] Shuichi T., Masatsugu N., Shigeharu K., Yasushige K., Toshinori M., Hideaki H., and Toshio Y., J. Phys. Chem. B 103, 5814 (1999).

[57] S.-H. Chen, J. Teixeira, and R. Nicklow, Phys. Rev. A 26, 3477 (1982).

[58] L. Toppozini, C. L. Armstrong, M. D. Kaye, M. Tyagi, T. Jenkins, and M. C. Rheinstaedter, ISRN Biophysics 2012, 1 (2012).

[59] W. Pfeiffer, T. Henkel, E. Sackmann, W. Knoll, and D. Richter, Europhys. Lett. 8, 201 (1989). 
[60] A. Lange, D. Marsh, K.-H. Wassmer, P. Meier, and G. Kothe, Biochemistry 24, $4383(1985)$.

[61] C. L. Armstrong, M. D. Kaye, M. Zamponi, E. Mamontov, M. Tyagi, T. Jenkins, and M. C. Rheinstaedter, Soft Matter 6, 5864 (2010).

[62] S. Busch, C. Smuda, L. C. Pardo, and T. Unruh, J. Am. Chem. Soc. 132, 3232 (2010).

[63] S. Konig, E. Sackmann, D. Richter, R. Zorn, C. Carlile, and T. M. Bayerl, J. Chem. Phys. 100, 3307 (1994).

[64] J. Fitter, R. E. Lechner, and N. A. Dencher, J. Phys. Chem. B 103, 8036 (1999).

[65] C. Faure, L. Bonakdar, and E. J. Dufourc, FEBS Lett. 405, 263 (1997).

[66] K. Wood, M. Plazanet, F. Gabel, B. Kessler, D. Oesterhelt, G. Zaccai, and M. Weik, Eur. Biophys. J. 37, 619 (2008).

[67] M. Weik, U. Lehnert, and G. Zaccai, Biophys. J. 89, 3639 (2005).

[68] C. Hamai, T. Yang, S. Kataoka, P. S. Cremer, and S. M. Musser, Biophys. J. 90, 1241 (2006).

[69] G. Binnig and C. F. Quate, Phys. Rev. 56, 930 (1986).

[70] N. Jalili and K. Laxminarayana, Mechatronics 14, 907 (2004).

[71] D. J. Muller and Y. F. Dufrene, Trends Cell Biol. 21, 461 (2011).

[72] H. Egawa and K. Furusawa, Langmuir 15, 1660 (1999). 
[73] L. Picas, P. E. Milhiet, and J. Hernandez-Borrell, Chem. Phys. Lipids 165, 845 (2012).

[74] H. M. Seeger, A. Di Cerbo, A. Allesandrini, and P. Facci, J. Phys. Chem. B 114, $8926(2010)$.

[75] G. van Meer and A. de Kroon, J. Cell Sci. 124, 5 (2011).

[76] W. Zhao, T. Rog, A. A. Gurtovenko, I. Vattulainen, and M. Karttunen, Biochimie 90, 930 (2008).

[77] W. van Klompenburg and B. de Kruijff, J. Membrane Biol. 162, 1 (1998).

[78] J. Pan, F. A. Heberle, S. Tristram-Nagle, M. Szymanski, M. Koepfinger, J. Katsaras, and N. Kucerka, Biochim. Biophys. Acta 1818, 2135 (2012).

[79] S. O. Diallo, E. Mamontov, A. Podlesnyak, G. Ehlers, N. Wada, S. Inagaki, and Y. Fukushima, J. Phys. Soc. Jpn. 82 (2013).

[80] K. O. Evans, Thin Solid Films 520, 3026 (2012).

[81] Y.-H. Kim, M. Rahman, Z.-L. Zhang, N. Misawa, R. Tero, and T. Urisu, Chem. Phys. Lett. 420, 569 (2006).

[82] R. Richter, A. Mukhopadhyay, and A. Brisson, Biophys. J. 85, 3035 (2003).

[83] L. Picas, C. Suarez-Germa, M. Teresa Montero, and J. Hernandez-Borrell, J. Phys. Chem. B 114, 3543 (2010).

[84] C. Loew, K. A. Riske, M. T. Lamy, and J. Seelig, Langmuir 27, 10041 (2011). 
[85] A. Watts, K. Harlos, W. Maschke, and D. Marsh, Biochim. Biophys. Acta 510, 63 (1978).

[86] S. Morandat, S. Azouzi, E. Beauvais, A. Mastouri, and K. El Kirat, Anal. Bioanal. Chem. 405, 1445 (2013).

[87] E. G. Finer and A. Darke, Chem. Phys. Lipids 12, 1 (1974).

[88] D.-K. Lee, B. S. Kwon, and A. Ramamoorthy, Langmuir 24, 13598 (2008).

[89] M. Bai, K. Knorr, M. J. Simpson, S. Trogisch, H. Taub, S. N. Ehrlich, H. Mo, U. G. Volkmann, and F. Y. Hansen, Europhysics Letters (EPL) 79, 26003 (2007).

[90] S. K. Wang, E. Mamontov, M. Bai, F. Y. Hansen, H. Taub, J. R. D. Copley, V. Garca Sakai, G. Gasparovic, T. Jenkins, M. Tyagi, K. W. Herwig, D. A. Neumann, W. Montfrooij, and U. G. Volkmann, EPL (Europhysics Letters) 91, 66007 (2010). 


\section{VITA}

Andrew Miskowiec was born in Syracuse, New York. He graduated from Worcester Polytechnic Institute in Worcester, MA in May, 2009 with a Bachelor of Science degree in Physics. He began his graduate work at the University of Missouri - Columbia in

June, 2009 under the advisement of Dr. Haskell Taub. In May, 2014, he graduated from the University of Missouri - Columbia with a Ph.D. in Physics and is now working at the Oak Ridge National Laboratory in Oak Ridge, TN. 